\title{
Search for squarks and gluinos in final states with one isolated lepton, jets, and missing transverse momentum at $\sqrt{s}=13 \mathrm{TeV}$ with the ATLAS detector
}

\author{
ATLAS Collaboration * \\ CERN, 1211 Geneva 23, Switzerland
}

Received: 6 January 2021 / Accepted: 17 June 2021 / Published online: 12 July 2021

(C) CERN for the benefit of the ATLAS collaboration 2021, corrected publication 2021

\begin{abstract}
The results of a search for gluino and squark pair production with the pairs decaying via the lightest charginos into a final state consisting of two $W$ bosons, the lightest neutralinos $\left(\tilde{\chi}_{1}^{0}\right)$, and quarks, are presented: the signal is characterised by the presence of a single charged lepton ( $e^{ \pm}$or $\mu^{ \pm}$) from a $W$ boson decay, jets, and missing transverse momentum. The analysis is performed using $139 \mathrm{fb}^{-1}$ of proton-proton collision data taken at a centre-of-mass energy $\sqrt{s}=13$ delivered by the Large Hadron Collider and recorded by the ATLAS experiment. No statistically significant excess of events above the Standard Model expectation is found. Limits are set on the direct production of squarks and gluinos in simplified models. Masses of gluino (squark) up to 2.2 (1.4) are excluded at 95\% confidence level for a light $\tilde{\chi}_{1}^{0}$.
\end{abstract}

\section{Introduction}

The Standard Model (SM) has proven to be a very successful theory. The discovery of the Higgs boson boson [1-4] by the ATLAS and CMS collaborations confirmed the predicted electroweak symmetry breaking, but also highlighted the hierarchy problem [5-8]. Supersymmetry (SUSY) [9-14] is a theoretical framework which assumes supersymmetric particles differing from their SM partners by a half unit of spin. By introducing a new fermionic (bosonic) supersymmetric partner for each boson (fermion) in the SM, SUSY provides a possible solution to the hierarchy problem. In SUSY models conserving $R$-parity [15], SUSY particles are produced in pairs. The lightest supersymmetric particle (LSP) has to be stable and is possibly weakly interacting, constituting a viable dark-matter candidate [16,17].

The partner particles of the SM fermions (quarks and leptons) are the scalar squarks $(\tilde{q})$ and sleptons $(\tilde{\ell})$. In the boson sector, the supersymmetric partners of the gluons are the

^e-mail: atlas.publications@ @ern.ch fermionic gluinos $(\tilde{g})$. The fermionic supersymmetric partners of the Higgs scalars (higgsinos) and of the electroweak gauge bosons (winos and bino) mix to form charged mass eigenstates (charginos) and neutral mass eigenstates (neutralinos). In the minimal supersymmetric extension of the Standard Model (MSSM) [18, 19], two scalar Higgs doublets along with their higgsino partners are necessary, resulting in two charginos $\left(\tilde{\chi}_{1,2}^{ \pm}\right)$and four neutralinos $\left(\tilde{\chi}_{1,2,3,4}^{0}\right)$.

Squarks and gluinos, in $R$-parity-conserving scenarios, are produced in pairs through the strong interaction. If strongly interacting gluinos or squarks are present at the scale, they should be produced copiously in the $13 p p$ collisions at the Large Hadron Collider (LHC). With the recorded integrated luminosity and the predicted cross-sections for squark and gluino production, the searches are expected to be sensitive to sparticle masses of a few .

This paper targets two simplified SUSY models $[20,21]$ describing gluino and first two generation squark $\left(\tilde{u}_{L}, \tilde{d}_{L}, \tilde{c}_{L}\right.$, $\tilde{s}_{L}$ ) production processes and decays. These models, introduced in Ref. [22], serve as benchmarks. In the models, referred to as the gluino and squark one-step models, gluinos or squarks are produced in pairs: gluinos subsequently decay via a virtual squark into a $\tilde{\chi}_{1}^{ \pm}$and two light quarks, while squarks decay into a $\tilde{\chi}_{1}^{ \pm}$and one light quark $(q \in\{u, d, s, c\})$. The $\tilde{\chi}_{1}^{ \pm}$then decay into a $W^{ \pm}$boson and a $\tilde{\chi}_{1}^{0}$. The corresponding diagrams are shown in Fig. 1. It is further assumed that $\tilde{\chi}_{1}^{ \pm}$is wino-like and the $\tilde{\chi}_{1}^{0}$ is bino-like. In both models, the branching fractions for SUSY particles are assumed to be $100 \%$ for the aforementioned processes squark/gluino decay into $\tilde{\chi}_{1}^{ \pm}$and quarks, and $\tilde{\chi}_{1}^{ \pm} \rightarrow \tilde{\chi}_{1}^{0} W^{ \pm}$. The SM particles are assumed to decay following their known branching fractions. All other sparticles, which do not explicitly appear in the decay chains, are set to be kinematically inaccessible and decoupled.

In this search, two different types of mass spectra are considered. In the first one, the $\tilde{\chi}_{1}^{ \pm}$mass is set to be exactly midway between the masses of the gluino (squark) and 
the $\tilde{\chi}_{1}^{0}$, so that the relative mass splitting $x=\left(m\left(\tilde{\chi}_{1}^{ \pm}\right)-\right.$ $\left.m\left(\tilde{\chi}_{1}^{0}\right)\right) /\left(m(\tilde{g} / \tilde{q})-m\left(\tilde{\chi}_{1}^{0}\right)\right)$ is equal to $1 / 2$. In the second mass spectrum, the $\tilde{\chi}_{1}^{0}$ mass is set to be $60 \mathrm{GeV}$ while the gluino (squark) mass and the relative mass splitting are free parameters.

The experimental signature of interest consists of a single charged lepton (electron or muon) produced by the leptonic decay of one of the $W$ bosons, at least two jets, and large missing transverse momentum ( $E_{\mathrm{T}}^{\text {miss }}$, defined in Sect. 4) from the undetected neutrino and the two neutralinos. The sparticle masses determine the energy available in their decays, so the number of jets and their kinematic properties depend on the mass spectrum chosen. To provide sensitivity to a broad range of mass spectra in the gluino and squark onestep models, four signal regions with differing jet multiplicity requirements from $\geq 2$ to $\geq 6$ are defined. Furthermore, the signal regions are categorised by allowing or forbidding the presence of jets originating from $b$ quarks ( $b$-tag and $b$-veto signal regions, respectively) to be sensitive to a wider class of decay processes, e.g. gluino decays producing top quarks.

The results presented in this paper are based on the ATLAS data collected in proton-proton collisions at the LHC during 2015-2018 at a centre-of-mass energy of 13 , corresponding to an integrated luminosity of $139 \mathrm{fb}^{-1}$. This analysis supersedes the previous ATLAS search with an integrated luminosity of $36.1 \mathrm{fb}^{-1}$ [23]. Similar searches for gluinos and squarks with decays via intermediate supersymmetric particles were performed by the CMS Collaboration [24,25].

\section{ATLAS detector}

The ATLAS detector [26-28] is a multipurpose particle detector with nearly $4 \pi$ coverage in solid angle. ${ }^{1}$ It consists of an inner tracking detector surrounded by a thin superconducting solenoid providing a $2 \mathrm{~T}$ axial magnetic field, electromagnetic and hadron calorimeters, and a muon spectrometer. The inner tracking detector covers the pseudorapidity range $|\eta|<2.5$. It consists of silicon pixel, silicon microstrip, and transition radiation tracking detectors. Lead/liquid-argon (LAr) sampling calorimeters provide electromagnetic (EM) energy measurements with high granularity. A steel/scintillator-tile hadron calorimeter covers the

\footnotetext{
${ }^{1}$ ATLAS uses a right-handed coordinate system with its origin at the nominal interaction point in the centre of the detector. The positive $x$ axis is defined by the direction from the interaction point to the centre of the LHC ring, with the positive $y$-axis pointing upwards, while the beam direction defines the $z$-axis. Cylindrical coordinates $(r, \phi)$ are used in the transverse plane, $\phi$ being the azimuthal angle around the $z$-axis. The pseudorapidity $\eta$ is defined in terms of the polar angle $\theta$ by $\eta=-\ln \tan (\theta / 2)$. Rapidity is defined as $y=0.5 \ln \left[\left(E+p_{z}\right) /\left(E-p_{z}\right)\right]$ where $E$ denotes the energy and $p_{z}$ is the component of the momentum along the beam direction. The angular distance $\Delta R$ is defined as $\sqrt{(\Delta y)^{2}+(\Delta \phi)^{2}}$.
}

central pseudorapidity range $(|\eta|<1.7)$. The endcap and forward regions are instrumented with LAr calorimeters for EM and hadronic energy measurements up to $|\eta|=4.9$. The muon spectrometer surrounds the calorimeters and is based on three large air-core toroidal superconducting magnets with eight coils each. The field integral of the toroids ranges between 2.0 and $6.0 \mathrm{Tm}$ across most of the detector. The muon spectrometer includes a system of precision tracking chambers and fast detectors for triggering. A twolevel trigger system [29] is used to select events. The firstlevel trigger is implemented in hardware and uses a subset of the detector information to keep the accepted rate below $100 \mathrm{kHz}$. This is followed by a software-based trigger that reduces the accepted event rate to $1 \mathrm{kHz}$ on average.

\section{Dataset and simulated events}

The search is performed using $139 \mathrm{fb}^{-1}$ of LHC $p p$ collision data collected between 2015 and 2018 by the ATLAS detector, with a centre-of-mass energy of 13 and a $25 \mathrm{~ns}$ proton bunch crossing interval. The average number of interactions per bunch crossing (pile-up) evolved over the datataking period from $\langle\mu\rangle=13$ in 2015 , to $\langle\mu\rangle=25$ in 2016, $\langle\mu\rangle=38$ in 2017 , and $\langle\mu\rangle=36$ in 2018 . The uncertainty in the combined 2015-2018 integrated luminosity is $1.7 \%$ [30], obtained using the LUCID-2 detector [31] for the primary luminosity measurements.

The SM background modelling, signal selection efficiencies, and signal event yield are evaluated using Monte Carlo (MC) simulated event samples. All the samples are produced by a fast simulation [32] procedure that combines a parameterisation of the calorimeter response with a GEANT4 [33] simulation of the other detector systems implemented in the ATLAS simulation infrastructure [34].

To model the pile-up observed in data, inelastic $p p$ events were generated with PYTHIA 8.186 [35] using the NNPDF2.3LO set of parton distribution functions (PDF) [36] and a set of tuned parameters called the A3 tune [37]. These events were overlaid on all simulated hard-scatter events to model the additional proton-proton interactions in the same and nearby bunch crossings. The pile-up overlay was reweighted to match the observed distribution in data. The simulated events are reconstructed with the same algorithms as used for data.

Signal gluino (squark) pair production samples were produced with MADGRAPH5_aMC@NLO v2.6.2 [38] at nextto-leading order for the hard-scattering matrix element and PYTHIA 8.212 (PYTHIA 8.230) for underlying event, parton shower and hadronization. Signal cross-sections are calculated to approximate next-to-next-to-leading order in the strong coupling constant, adding the resummation of soft gluon emission at next-to-next-to-leading-logarithm accu- 
Fig. 1 Diagrams for a gluino and $\mathbf{b}$ squark pair production with subsequent decays into quarks and a $\tilde{\chi}_{1}^{ \pm}$. The $\tilde{\chi}_{1}^{ \pm}$ decays into a $\tilde{\chi}_{1}^{0}$ and a $W$ boson. This analysis targets final states in which one $W$ decays leptonically and the other hadronically

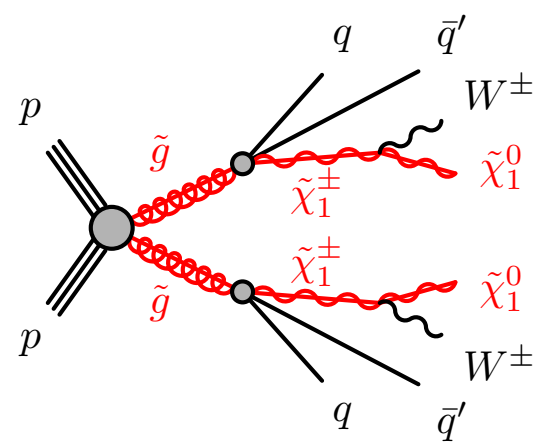

(a)

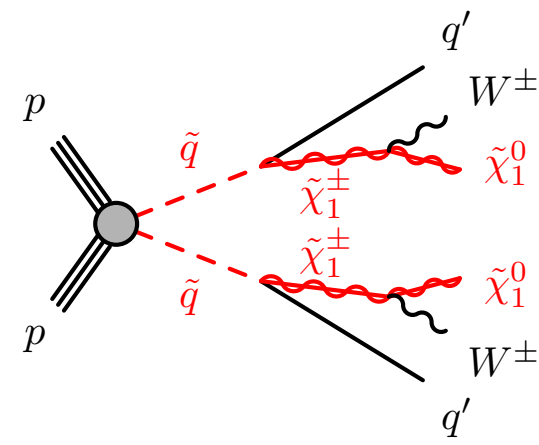

(b) racy (approximate NNLO+NNLL) [39-46]. The nominal cross-section and its uncertainty are derived using the PDF4LHC15_mc PDF set, following the recommendations of Ref. [47]. A typical cross-section for gluino production with $m_{\tilde{g}}=2000 \mathrm{GeV}$, and $m \tilde{\chi}_{1}^{0}=200 \mathrm{GeV}$ is $1.01 \pm 0.20 \mathrm{fb}$, while for squarks with $m_{\tilde{q}}=1200 \mathrm{GeV}$ and $m \tilde{\chi}_{1}^{0}=200 \mathrm{GeV}$ a typical cross-section is $6.8 \pm 0.9 \mathrm{fb}$ when the four partners of the left-handed first two generation quarks $\left(\tilde{u}_{\mathrm{L}}, \tilde{d}_{\mathrm{L}}, \tilde{s}_{\mathrm{L}}\right.$, and $\tilde{c}_{\mathrm{L}}$ ) are assumed to be mass-degenerate. A 'single squark flavour' limit is also given assuming that only one such lefthanded first and second generation quarks is kinematically accessible.

All relevant SM backgrounds are considered: $t \bar{t}$ pair production; single-top production ( $s$-channel, $t$-channel, and associated $W t$ production); $W / Z+$ jets production; $t \bar{t}$ production with an electroweak boson $(t \bar{t}+V)$; and diboson ( $W W, W Z, Z Z$ ) production. Different MC event generators were used to produce the background samples, depending on their production process. The MC-produced events are then normalised to data using the corresponding theoretical cross-sections. The event generators, the routines for parton showering and hadronisation, and the parameter tunes and parton distribution functions for all background processes produced are summarised in Table 1.

The $W+$ jets events were generated using SHERPA: the generation process includes up to two partons at NLO and four partons at LO using Comix [48] and OpenLoops [49, 50]. The matrix element was merged with the SHERPA parton shower [51] according to the ME+PS@NLO prescription [52-55] using the set of tuned parameters developed by the SHERPA authors. To simulate the properties of the bottom- and charmhadron decays, the EvtGen v1.2.0 [56] program was used for all samples showered with PYTHIA.

Systematic uncertainties, for both signal and background samples, derived from the MC generator configuration are evaluated using samples produced without detector simulation. The uncertainties account for variations of the renormalisation and factorisation scales, the CKKW-L [57] matching scale, as well as different PDF sets and fragmenta- tion/hadronisation models. Details of the MC modelling uncertainties are discussed in Sect. 7.

\section{Object reconstruction}

Each event is required to have at least one reconstructed interaction vertex with a minimum of two associated tracks, each having $p_{\mathrm{T}}>500$. In events with multiple vertices, the one with the highest sum of squared transverse momenta of associated tracks is chosen as the primary vertex (PV) [72]. Baseline quality criteria are applied to reject events with noncollision backgrounds or detector noise [73].

Two levels of object definition for leptons and jets are used: 'baseline' and 'signal'. Loose quality requirements define baseline objects, which are used in the calculation of missing transverse momentum and in the overlap removal procedure described below. Signal objects, obtained by applying more selective identification criteria to objects passing the baseline requirements, are used as input for the actual search region definitions. Isolation criteria applied to a set of track-based and calorimeter-based variables, are used to discriminate between signal leptons and semileptonic heavy-flavour decays, photon conversions as well as jets misidentified as leptons.

Energy deposits in the electromagnetic (EM) calorimeter that are matched to charged-particle tracks in the inner detector (ID) [74] provide electron candidates. The $p_{\mathrm{T}}$ of electron is calculated based on the energy deposited in the EM calorimeter. Baseline electrons must have $p_{\mathrm{T}}>7 \mathrm{GeV}$ and $|\eta|<2.47$ and must satisfy the Loose working point provided by a likelihood-based algorithm, described in Ref. [74]. The longitudinal impact parameter $^{2} z_{0}$ relative to the

\footnotetext{
2 The longitudinal impact parameter $z_{0}$ corresponds to the $z$-coordinate distance between the point along the track at which the transverse impact parameter is defined and the primary vertex. The transverse impact parameter $d_{0}$ is defined as the distance of closest approach in the transverse plane between a track and the beam-line. The uncertainty in $d_{0}$ is denoted $\sigma\left(d_{0}\right)$.
} 
Table 1 Overview of MC generators used for different simulated event samples for background

\begin{tabular}{llllll}
\hline Process & Generator & $\begin{array}{c}\text { Parton shower } \\
\text { and } \\
\text { hadronisation }\end{array}$ & Tune & PDF & Cross-section \\
\hline$t \bar{t}$ & PowHEG- BoX v2 & $\begin{array}{c}\text { PYTHIA 8.230 } \\
\text { [62] }\end{array}$ & A14 [63] & NNPDF2.3LO [36] & NNLO+NNLL [64] \\
Single top & POWHEG- BoX v2 & PYTHIA 8.230 & A14 & NNPDF2.3LO & NLO+NNLL [68] \\
$W / Z+$ jets & SHERPA 2.2.1 [69] & SHERPA 2.2.1 & SHERPA default & NNPDF3.0NNLO & NNLO [70] \\
Diboson & SHERPA 2.2.1 and & $\begin{array}{c}\text { SHERPA 2.2.1 } \\
\text { and 2.2.2 }\end{array}$ & SHERPA default & NNPDF3.0NNLO & NLO \\
$t \bar{t}+V$ & 2.2 .2 & PYTHIA 8.210 & A14 & NNPDF2.3LO & NLO [71] \\
\hline
\end{tabular}

$\mathrm{PV}$ is required to satisfy $\left|z_{0} \sin \theta\right|<0.5 \mathrm{~mm}$. The number of hits on the track is used to discriminate between electrons and converted photons. Signal electron candidates are required to satisfy the Tight likelihood operating point and the requirement $\left|d_{0} / \sigma\left(d_{0}\right)\right|<5$. The Loose and HighPtCaloOnly isolation working points, described in Ref. [74], are applied to signal electrons having $p_{\mathrm{T}}<200 \mathrm{GeV}$ and $p_{\mathrm{T}}>200 \mathrm{GeV}$, respectively.

Signal electrons with $p_{\mathrm{T}}<200 \mathrm{GeV}$ are refined using the Loose isolation working point, while those with larger $p_{\mathrm{T}}$ are required to pass the HighPtCaloOnly isolation working point, as described in Ref. [74].

Muon candidates are reconstructed from matching tracks in the ID and muon spectrometer, refined through a global fit using the hits from both subdetectors [75]. Baseline muons are required to satisfy $p_{\mathrm{T}}>6 \mathrm{GeV}$ and $|\eta|<2.7$. They are identified using the Medium identification criteria [75]. As with the electrons, baseline muons are required to satisfy $\left|z_{0} \sin \theta\right|<0.5 \mathrm{~mm}$. Signal muon candidates must also satisfy tighter pseudorapidity and transverse impact parameter requirements, $|\eta|<2.5$ and $\left|d_{0} / \sigma\left(d_{0}\right)\right|<3$, and the FixedCutLoose isolation working point requirements.

Jet candidates are reconstructed from three-dimensional topological energy clusters in the calorimeters using the anti$k_{t}$ algorithm [76] with a radius parameter $R=0.4$ [77]. Baseline jets must have $|\eta|<4.5$ and $p_{\mathrm{T}}>20 \mathrm{GeV}$. To suppress pile-up interactions, those jets having $|\eta|<2.8$ and $p_{\mathrm{T}}<120 \mathrm{GeV}$ are required to pass the Medium working point of the jet vertex tagger (JVT), a multivariate algorithm that identifies jets originating from the PV using track information $[78,79]$. Signal jets must also have $|\eta|<2.8$ and $p_{\mathrm{T}}>30 \mathrm{GeV}$.

Jets with $p_{\mathrm{T}}>20 \mathrm{GeV}$ in the region $|\eta|<2.5$ that contain $b$-hadrons can be ' $b$-tagged' with high efficiency by the MV2c10 [80], which is a boosted decision tree with improved light-flavour jet and $c$-jet rejection. The $b$-tagging working point provides an efficiency of $77 \%$ for jets containing $b$-hadrons in simulated $t \bar{t}$ events, with rejection fac- tors of 110 and 4.9 for light-flavour jets and jets containing $c$-hadrons, respectively [81]. Signal $b$-jets should also have $p_{\mathrm{T}}>30 \mathrm{GeV}$.

An overlap removal procedure is applied to the baseline objects defined above to resolve reconstruction ambiguities between electrons, muons and jets. First, any electron sharing the same ID track with a muon is rejected. If two electrons share the same ID track, the one with lower $p_{\mathrm{T}}$ is discarded. Next, jets are rejected if they lie within $\Delta R=0.2$ of an electron and then electrons are removed if they are within a cone of $p_{\mathrm{T}}$-dependent size $\Delta R=\min \left(0.4,0.04+10 \mathrm{GeV} / p_{\mathrm{T}}\right)$ around a jet. Subsequently, jets are rejected if they are within $\Delta R=0.2$ of a muon or if the muon is matched to the jet through ghost association [82]. Finally, muons within a cone, defined in the same way as for electrons, around any remaining jet are removed.

The missing transverse momentum, $\boldsymbol{p}_{\mathrm{T}}^{\text {miss }}$, with magnitude, $E_{\mathrm{T}}^{\text {miss }}$, is calculated as the negative vectorial sum of the transverse momenta of all reconstructed baseline objects (electrons, muons, jets and photons [83]) and a soft term. The soft term includes all selected tracks associated with the PV but not matched to any reconstructed baseline object. To suppress contributions from pile-up and improve the $E_{\mathrm{T}}^{\text {miss }}$ resolution, tracks not associated with the PV are excluded from the $E_{\mathrm{T}}^{\mathrm{miss}}$ calculation $[84,85]$.

The efficiency differences in the trigger, lepton identification and reconstruction between data and simulated events are closely evaluated in independent measurements, and are accounted for by applying the corresponding corrections to the simulation in this analysis.

\section{Event selection}

To retain acceptance for soft leptons, events satisfying the $E_{\mathrm{T}}^{\text {miss }}$ trigger selection were recorded [86] and used in the search. The trigger efficiency is higher than $98 \%$ for offline $E_{\mathrm{T}}^{\text {miss }}$ values above $250 \mathrm{GeV}$. To target the signal-like events, selected events are required to have exactly one signal lepton, 
either an electron or a muon. Events with additional baseline leptons are rejected to suppress dilepton $t \bar{t}$, single-top (Wtchannel), $Z+$ jets and diboson backgrounds. The following observables are used to further reduce background contributions and increase the sensitivity for signal:

- The transverse mass, $m_{\mathrm{T}}$, is defined from the lepton transverse momentum $\boldsymbol{p}_{\mathrm{T}}^{\ell}$ and $\boldsymbol{p}_{\mathrm{T}}^{\text {miss }}$ as

$m_{\mathrm{T}}=\sqrt{2 p_{\mathrm{T}}^{\ell} E_{\mathrm{T}}^{\mathrm{miss}}\left(1-\cos \left[\Delta \phi\left(\boldsymbol{p}_{\mathrm{T}}^{\ell}, \boldsymbol{p}_{\mathrm{T}}^{\text {miss }}\right)\right]\right)}$,

where $\Delta \phi\left(\boldsymbol{p}_{\mathrm{T}}^{\ell}, \boldsymbol{p}_{\mathrm{T}}^{\text {miss }}\right)$ is the azimuthal angle between $\boldsymbol{p}_{\mathrm{T}}^{\ell}$ and $\boldsymbol{p}_{\mathrm{T}}^{\text {miss }}$. It has an upper endpoint at the $W$ boson mass for $W+$ jets events and for semileptonic $t \bar{t}$ events. The $m_{\mathrm{T}}$ distribution for signal events extends significantly beyond that endpoint.

- The effective mass, $m_{\mathrm{eff}}$, is the scalar sum of the $p_{\mathrm{T}}$ of the signal lepton and all signal jets and $E_{\mathrm{T}}^{\text {miss }}$ :

$m_{\mathrm{eff}}=p_{\mathrm{T}}^{\ell}+\sum_{j=1}^{N_{\mathrm{jet}}} p_{\mathrm{T}, j}+E_{\mathrm{T}}^{\mathrm{miss}}$.

The effective mass provides good discrimination against background events, especially for the signal scenarios with energetic jets. It can also help to distinguish between different signal channels. For example gluino production shows higher jet multiplicity than squark production. High-mass gluinos and squarks are expected to produce harder jets than low-mass ones. Thus, the optimal $m_{\text {eff }}$ value depends on the different signal scenarios. To achieve a wide-range sensitivity to various SUSY models with a limited number of signal regions, multiple intervals in $m_{\text {eff }}$ are used in the final model-dependent signal regions.

- The aplanarity is a variable designed to provide more global information about the full momentum tensor of the event. It is constructed from the lepton and the jets, and is defined as $(3 / 2) \times \lambda_{3}$, where $\lambda_{3}$ is the smallest eigenvalue of the sphericity tensor [87]. Typical measured aplanarity values lie in the range $0-0.3$, with values near zero indicating highly planar background-like events. Strongly produced SUSY signals tend to have high aplanarity values, since they are more spherical than background events due to the multiple objects emitted in the gluino/squark decay chains.

Four mutually exclusive signal regions (SRs) are designed to enhance the signal sensitivity. The selection criteria for the four SRs are summarised in Table 2. Each SR is optimised for specific SUSY scenarios, as discussed below. They are labelled by the minimum required number of jets and, where relevant, the characteristics of the targeted supersymmetric mass spectrum: 2J, 4J high-x, 4J low-x, and 6J. When setting model-dependent exclusion limits ('excl'), each SR is divided in $m_{\text {eff }}$ intervals and in $b$-veto/ $b$-tag categories, and a simultaneous fit is performed across all bins of the four SRs. This choice enhances the sensitivity to a range of new-physics scenarios with or without $b$-quarks in the final states, and with different mass splittings. For model-independent limits and null-hypothesis tests ('disc' for discovery), the event yield in each SR is used to search for an excess over the SM background using an optimised minimum $m_{\text {eff }}$ value. The discovery SRs require the $b$-veto and are separately optimised for gluino and squark cases. The systematic uncertainties, fits, and results discussed in the following sections for the simplfied models are based on the exclusion SRs, while the model-independent results are based on the discovery SRs.

The 2J SR targets compressed scenarios where differences between $m_{\tilde{g} / \tilde{q}}, m \tilde{\chi}_{1}^{ \pm}$, and $m \tilde{\chi}_{1}^{0}$ are small and the decay products tend to have low $p_{\mathrm{T}}$. Thus, events are required to have one low- $p_{\mathrm{T}}$ lepton and at least two jets. The lower $p_{\mathrm{T}}^{\ell}$ bound is 7 (6) $\mathrm{GeV}$ for the electron (muon), and the upper $p_{\mathrm{T}}^{\ell}$ bound increases with the jet multiplicity up to $25 \mathrm{GeV}$. The upper $p_{\mathrm{T}}^{\ell}$ requirement ensures the orthogonality between the $\mathbf{2} \mathbf{J}$ SR and other signal regions. The jet multiplicity dependence maintains the balance between background rejection and signal acceptance: the leptons are more energetic for signals with increasing mass splittings. Stringent requirements are placed on $E_{\mathrm{T}}^{\text {miss }}$ and on $E_{\mathrm{T}}^{\text {miss }} / m_{\text {eff }}$ to enhance the signal sensitivity by selecting signal events with boosted final-state neutralinos recoiling against energetic initial-state radiation (ISR) jets. Compared to other SRs, a less stringent lower bound on $m_{\text {eff }}$ is preferred.

The 4J high-x SR provides sensitivity to the models with a fixed $m \tilde{\chi}_{1}^{0}$ of $60 \mathrm{GeV}$ and a high $x$ value, i.e. when $m \tilde{\chi}_{1}^{ \pm}$ and $m_{\tilde{g} / \tilde{q}}$ are relatively close. Events with four or five jets are selected for this scenario. The mass-splitting between $m \tilde{\chi}_{1}^{ \pm}$ and $m \tilde{\chi}_{1}^{0}$ is large enough to produce a boosted $W$ boson that decays into a high- $p_{\mathrm{T}}$ lepton and a neutrino. Large $m_{\mathrm{T}}$ is thus the most distinguishing characteristic of this SR. Relatively soft jets are expected to be emitted from the gluino or squark decays. The SM background is further suppressed by tight requirements on $E_{\mathrm{T}}^{\text {miss }}$, aplanarity, and $E_{\mathrm{T}}^{\text {miss }} / m_{\mathrm{eff}}$. Compared to the 2J SR, a tighter $m_{\text {eff }}$ selection is applied due to higher jet activity.

The 4J low-x SR is optimised for the models where $m \tilde{\chi}_{1}^{0}$ is fixed to $60 \mathrm{GeV}$ and $x \approx 0$, i.e. $m \tilde{\chi}_{1}^{ \pm}$is close to $m \tilde{\chi}_{1}^{0}$. The jet multiplicity requirement is the same as in the $\mathbf{4 J}$ high-x SR. In contrast to the high- $x$ scenarios, the small mass-splitting between $m \tilde{\chi}_{1}^{ \pm}$and $m \tilde{\chi}_{1}^{0}$ tends to produce an off-shell $W$ boson, leading to small $m_{\mathrm{T}}$. To keep this SR orthogonal to the $\mathbf{4 J}$ high-x SR, an upper bound is applied to $m_{\mathrm{T}}$. Other than that, the requirements on $m_{\mathrm{eff}}, E_{\mathrm{T}}^{\mathrm{miss}}$, aplanarity, and $E_{\mathrm{T}}^{\mathrm{miss}} / m_{\mathrm{eff}}$ are identical to the ones used in the $\mathbf{4 J}$ high-x SR. 
Table 2 Overview of the selection criteria for the signal regions used for gluino/squark one-step models. The requirements that only apply to the exclusion (discovery) SRs are marked with 'excl' ('disc'). The $m_{\text {eff }}$ bins are of even width and the ' + ' indicates that overflow events are included in the last bin

\begin{tabular}{|c|c|c|c|c|}
\hline SR & $\mathbf{2 J}$ & 4J high-x & 4J low-x & 6J \\
\hline$N_{\ell}$ & \multicolumn{4}{|c|}{$=1$} \\
\hline$p_{\mathrm{T}}^{\ell}[\mathrm{GeV}]$ & $\begin{array}{l}>7(6) \text { for } e(\mu) \text { and } \\
<\min \left(10 \cdot N_{\mathrm{jet}}, 25\right)\end{array}$ & $>25$ & $>25$ & $>25$ \\
\hline$N_{\text {jet }}$ & $\geq 2$ & $4-5$ & $4-5$ & $\geq 6$ \\
\hline$p_{T}^{\mathrm{jet}}[\mathrm{GeV}]$ & $>30$ & $>30$ & $>30$ & $>30$ \\
\hline$E_{\mathrm{T}}^{\text {miss }}[\mathrm{GeV}]$ & $>400$ & $>300$ & $>300$ & $>300$ \\
\hline$m_{\mathrm{T}}[\mathrm{GeV}]$ & $>100$ & $>520$ & $150-520$ & $>225$ \\
\hline Aplanarity & - & $>0.01$ & $>0.01$ & $>0.05$ \\
\hline$E_{\mathrm{T}}^{\mathrm{miss}} / m_{\mathrm{eff}}$ & $>0.25$ & $>0.2$ & $>0.2$ & - \\
\hline$N_{b \text {-jet }}(\mathrm{excl})$ & \multicolumn{4}{|c|}{$=0$ for the $b$-veto SR, $\geq 1$ for the $b$-tag SR } \\
\hline$m_{\mathrm{eff}}[\mathrm{GeV}](\mathrm{excl})$ & 3 bins $\in[700,2500+]$ & 3 bins $\in[1000,2800+]$ & 3 bins $\in[1000,2800+]$ & 4 bins $\in[700,3500+]$ \\
\hline$N_{b \text {-jet }}($ disc $)$ & \multicolumn{4}{|c|}{$=0$} \\
\hline$m_{\mathrm{eff}}[\mathrm{GeV}]($ disc $)$ & $\begin{array}{l}>1900 \text { (1300) for gluino } \\
\text { (squark) }\end{array}$ & $>2200$ & $>2200$ & $\begin{array}{l}>2800 \text { (2100) for } \\
\text { gluino (squark) }\end{array}$ \\
\hline
\end{tabular}

Table 3 Overview of the control region selection criteria. The top and $W+$ jets control regions are defined by the $b$-tag and $b$-veto requirements, respectively. The $m_{\mathrm{eff}}$ bins are of even width and the ' + ' indicates that overflow events are included in the last bin

\begin{tabular}{llll}
\hline CR & 2J & 4J & 6J \\
\hline$N_{\ell}$ & & $=1$ & \\
$p_{\mathrm{T}}^{\ell}[\mathrm{GeV}]$ & $>7(6)$ for $e(\mu)$ and & $>25$ & $>25$ \\
& $<\min \left(10 \cdot N_{\text {jet }}, 25\right)$ & & $\geq 6$ \\
$N_{\text {jet }}$ & $\geq 2$ & $4-5$ & $>250$ \\
$E_{\mathrm{T}}^{\text {miss }}[\mathrm{GeV}]$ & $>400$ & $>300$ & $50-100$ \\
$m_{\mathrm{T}}[\mathrm{GeV}]$ & $50-80$ & $50-90$ & $>0.05$ \\
Aplanarity & - & $>0.01$ & - \\
$E_{\mathrm{T}}^{\text {miss }} / m_{\text {eff }}$ & $>0.25$ & $>0.2$ & 4 bins $\in[700,3500+]$ \\
$m_{\text {eff }}[\mathrm{GeV}]$ & 3 bins $\in[700,2500+]$ & 3 bins $\in[1000,2800+]$ & \\
$N_{b \text {-jet }}$ & & $\geq 1$ for the top CR; $=0$ for the $W+$ jets CR \\
\hline
\end{tabular}

The 6J SR targets signal scenarios with high gluino/squark mass, and is optimised for models with $x \approx 1 / 2$. Events with one high- $p_{\mathrm{T}}$ lepton and at least six jets are selected. Large aplanarity is required, reflecting the heavy gluino/squark produced in the targeted signature. Tight requirements on $m_{\mathrm{T}}$ and $E_{\mathrm{T}}^{\text {miss }}$ are imposed to reduce the SM background. To achieve high sensitivity for a wide range of $m_{\tilde{g} / \tilde{q}}$, four exclusive bins are defined in $m_{\text {eff }}$ and used in the fit. The lowest mass bin starts from $700 \mathrm{GeV}$, and the highest from $2800 \mathrm{GeV}$.

\section{Background estimation}

Robust prediction of the SM background event yields in SRs is important in any search like the one presented in this paper.
Different approaches for calculating the SM event yields in the SRs are used depending on the background process of interest. The yields of $t \bar{t}$, single-top, and $W+$ jets processes are estimated from data using a set of dedicated control regions (CRs), while contributions from $Z+$ jets, $t \bar{t}$ produced in association with a $W$ or $Z$ boson, and dibosons ( $W W$, $W Z, Z Z)$ are evaluated from MC simulation. The details are described below.

Three sets of CRs, $\mathbf{2 J}, \mathbf{4 J}, \mathbf{6 J}$, are defined for estimating the backgrounds in $\mathbf{2} \mathbf{J}, \mathbf{4 J}$ high-x, $\mathbf{4 J}$ low-x and $\mathbf{6 J}$ signal regions. The CRs satisfy the criteria of high purity for the targeted background process and low signal contamination from the model of interest. The purity varies from 57 to $88 \%$ for the top backgrounds ( $t \bar{t}$ and single top) in top CRs and from 73 to $92 \%$ for $W+$ jets in $W+$ jets CRs. Each of the 
Fig. 2 The $m_{\mathrm{T}}$ distributions in the signal regions after all of the selection requirements other than the $m_{\mathrm{T}}$ cut (noted as ' $\mathrm{N}-1$ ' in the figures). Due to the removal of the $m_{\mathrm{T}}$ requirement, these plots effectively show the CRs, VRs and SRs for each jet multiplicity. The uncertainty bands include all statistical and systematic uncertainties.

Overflow events are included in the last bin. The dashed lines represent benchmark signal points for gluino and squark pair production
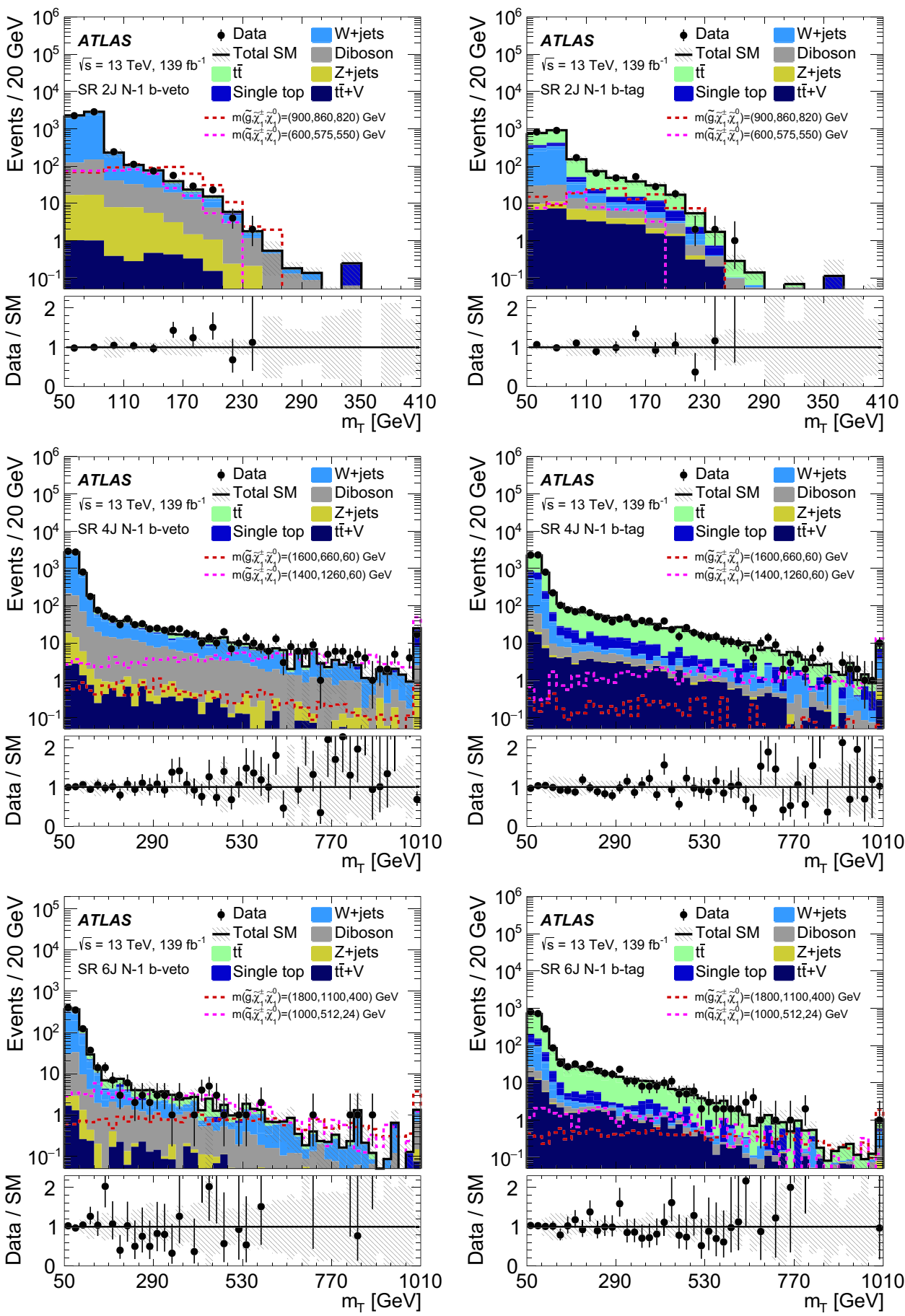

CRs is defined with kinematic boundaries close to the corresponding SR in order to reduce the theoretical and detector uncertainties from the extrapolation. The contributions of the top and $W+$ jets backgrounds in the SRs are evaluated with a fit based on the profile likelihood method. The normalised background predictions are obtained from a simultaneous fit across all control regions, as described in Sect. 8. The control regions for top and $W+$ jets are presented in Table 3 . Events in the top control region require at least one $b$-tagged signal jet in the event, while $W+$ jets control regions are defined by vetoing all events containing any $b$-tagged signal jets. The CRs are crafted in the same way as signal regions, thus each $\mathrm{CR}$ is defined as a function of $m_{\text {eff }}$, with the same binning as the corresponding SR. This permits extrapolation from each $b$-tag/ $b$-veto and $m_{\text {eff }}$ bin in CRs to the corresponding bin in the SRs. The extrapolation from CRs to SRs is performed via the $m_{\mathrm{T}}$ variable, which is found to be well modelled in simulation as shown in Fig. 2. 
Table 4 Overview of the validation region selection criteria. The top and $W+$ jets validation regions are defined by the $b$-tag and $b$-veto requirements, respectively. The $m_{\text {eff }}$ bins are of even width and the ' + ' indicates that overflow events are included in the last bin

\begin{tabular}{llll}
\hline VR & 2J & 4J & 6J \\
\hline$N_{\ell}$ & & $=1$ & \\
$p_{\mathrm{T}}^{\ell}[\mathrm{GeV}]$ & $>7(6)$ for $e(\mu)$ and & $>25$ & $>25$ \\
& $<\min \left(10 \cdot N_{\text {jet }}, 25\right)$ & & $\geq 6$ \\
$N_{\text {jet }}$ & $\geq 2$ & $4-5$ & $>250$ \\
$E_{\mathrm{T}}^{\text {miss }}[\mathrm{GeV}]$ & $>400$ & $>300$ & $100-225$ \\
$m_{\mathrm{T}}[\mathrm{GeV}]$ & $80-100$ & $90-150$ & $>0.05$ \\
Aplanarity & - & $>0.01$ & - \\
$E_{\mathrm{T}}^{\text {miss }} / m_{\mathrm{eff}}$ & $>0.25$ & $>0.2$ & 3 bins $\in[700,2800+]$ \\
$m_{\text {eff }}[\mathrm{GeV}]$ & 3 bins $\in[700,2500+]$ & 3 bins $\in[1000,2800+]$ & \\
$N_{b \text {-jet }}$ & & $\geq 1$ for the top VR; $=0$ for the $W+$ jets VR
\end{tabular}

In order to validate the background fit results, cross-checks of the background estimates are performed in validation regions (VRs) situated between the SRs and the CRs in $m_{\mathrm{T}}$, while remaining orthogonal to both regions. The VRs are also defined as functions of $m_{\text {eff }}$ in the same way as the corresponding CRs and SRs, to ensure an $m_{\text {eff }}$-dependent validation. The highest $m_{\text {eff }}$ bin in the $\mathbf{6 J}$ VR is not used because its signal contamination would be too large. Similar to the control regions, events in the top VRs require a $b$-tag, while events in the $W+$ jets VRs require a $b$-veto. The VRs are not used to constrain the fit; they serve only to verify that the normalised background predictions agree with the observed data. The VR definitions and their graphical representation are shown in Table 4 and Fig. 3 .

The background contributions from $Z+$ jets, $t \bar{t}+V$ and diboson events are evaluated from simulation. The simulated event samples are normalised to the relevant theoretical cross-sections. No dedicated control regions for the diboson background are used, as the modelling of this background by simulation is found to be sufficiently good when compared with the data in the validation regions. The data and MC predictions yield, obtained from the overall background estimate, differ in all validation regions by less than two standard deviations. The background originating from misidentified leptons, real leptons coming from jets produced by heavyflavour quarks or photons converted to electrons is evaluated using both MC and data-driven methods, and it is found to be negligible within the statistical error of the data due to the stringent requirements on $E_{\mathrm{T}}^{\text {miss }}$.

As a representative example, the $m_{\text {eff }}$ distributions in the 6J top and $W+$ jets control regions are shown in Fig. 4 before and after a fit which constrains only the control regions. The fit strategy is discussed in Sect. 8. A trend is observed, as it was in previous similar searches [23], whereby the MC overestimates the expected yields at large values of $m_{\mathrm{eff}}$. This is accounted for by applying different normalisation parameter values for each $m_{\text {eff }}$ bin in the corresponding fit, which effectively corrects the mismodelling. In the post-fit distributions, the data and the background expectation agree well within the uncertainties.

\section{Systematic uncertainties}

The expected yields for both the signal and background events are subject to theoretical and experimental systematic uncertainties. The theoretical uncertainties for the backgrounds normalised to data influence only the transfer factors from $\mathrm{CR}(\mathrm{s})$ to $\mathrm{VR}(\mathrm{s})$ and from $\mathrm{CR}(\mathrm{s})$ to $\mathrm{SR}(\mathrm{s})$, while for the other backgrounds, the uncertainties affect the inclusive cross-section of each process and the acceptance of the analysis selection.

Following the procedure described in Sect. 6, both the experimental and the theoretical uncertainties are computed separately for each $m_{\text {eff }}$ bin. Both theoretical and experimental uncertainties are correlated across all $m_{\text {eff }}$ bins. For the single-top and $t \bar{t}$ backgrounds, the theoretical uncertainties due to parton shower and hadronisation/fragmentation are estimated by comparing the predictions obtained with the POWHEG- BOX generator interfaced with two different parton shower generators, PYTHIA 8 and HERWIG 7 [88], while those due to the hard-scattering are evaluated by a POWHEGBOX + PYTHIA 8 to MG5_aMC@NLO + PYTHIA 8 comparison. Diagram removal (DR) and diagram subtraction (DS) samples, modelled by POWHEG- BOX + PYTHIA 8, are used to determine the impact of the interference between single-top $W t$ and $t \bar{t}$ production [89]. In order to evaluate the impact of the uncertainties coming from the emission of initial- and final-state radiation, the renormalisation, and factorisation scales and showering are varied.

Uncertainties for $t \bar{t}+V, W / Z+$ jets and dibosons coming from scale variations are evaluated by considering the envelope of the seven-point variations of the renormalisation and factorisation scales. The resummation and the CKKW 
Fig. 3 Graphical illustration of the control and validation region configuration corresponding to the $\mathbf{2 J}$ (top left), $\mathbf{4 J}$ (top right), and $\mathbf{6 J}$ (bottom) regions. The variables shown on the horizontal and vertical axes indicate the selections that differ between the corresponding control regions, validation regions and signal regions. The dotted lines show the boundaries of the $m_{\mathrm{eff}}$ binning of the exclusion SRs
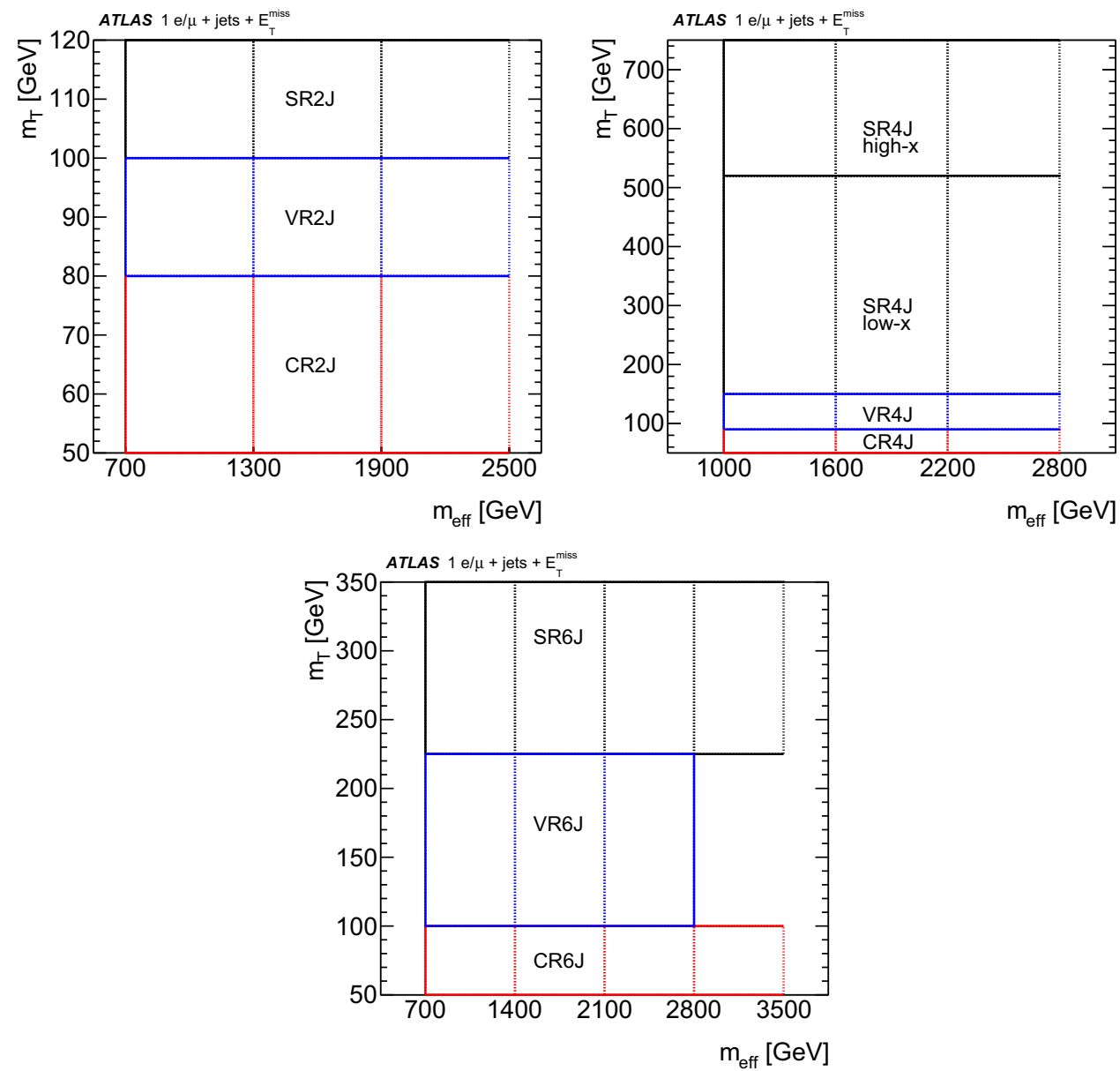

matching variations for $W / Z+$ jets are estimated by varying the corresponding scale parameters up and down by a factor of two relative to the nominal value for each region. The PDF uncertainties for $W / Z+$ jets are considered following the recommendation in PDF4LHC15 [47], while those for $t \bar{t}$ were found to be negligible in all the regions. Systematic uncertainties of $5 \%$ and $6 \%$ are assigned to the inclusive cross-sections of the $t \bar{t}+V$ and diboson processes [90], respectively. For the other background processes such as $Z+$ jets, a systematic uncertainty in the inclusive cross-section is included at the $5 \%$ level.

The theoretical uncertainties in the expected yields for the two signal models are considered and estimated using MG5_aMC@NLO + PYTHIA 8 samples by varying the parameters corresponding to the factorisation, renormalisation and CKKW-L matching scales.

Detector-related systematic uncertainties include uncertainties from jet energy scale (JES), jet energy resolution (JER), lepton reconstruction and identification, $b$-tagging, $E_{\mathrm{T}}^{\text {miss }}$ modelling, pile-up, and the trigger efficiency. The dominant experimental systematic uncertainties stem from the JES and JER uncertainties. They are derived as a function of $p_{\mathrm{T}}$ and $\eta$ of the jet, the pile-up conditions and the jet flavour composition [91]. The uncertainties due to the lepton identification, momentum/energy scale and resolution are estimated from samples of $Z \rightarrow \ell^{+} \ell^{-}, J / \psi \rightarrow \ell^{+} \ell^{-}$ and $W \rightarrow \ell v$ decays $[74,75]$. The $E_{\mathrm{T}}^{\text {miss }}$ modelling systematic uncertainties are evaluated by accounting for the uncertainties in the energy and momentum scale of each object used in the calculation, as well as the uncertainties in the soft term's resolution and scale. The uncertainty due to pile-up modelling is computed by varying the reweighting factor by $\pm 4 \%$. Uncertainties related to the $b$-tagging efficiency are derived from data-driven measurements in $t \bar{t}$ events [80,92], while uncertainties associated with the probability of mistakenly $b$-tagging a jet which does not contain a $b$-hadron are determined using dijet samples [93]. The uncertainties in the dominant background normalisation are obtained when performing the fit including the background control regions.

Tables 5 and 6 detail the size of the different systematic uncertainties in the signal regions, summed over all $m_{\text {eff }}$ bins. The uncertainty in the hard-scattering for $t \bar{t}$ dominates in many regions. The determination with Monte Carlo samples 
Fig. 4 The $m_{\text {eff }}$ distribution in 6J top (left, labelled as 'TR') and $W+$ jets (right, labelled as 'WR') control regions before (top) and after (bottom) the fit. The uncertainty bands include all statistical and systematic uncertainties. Overflow events are included in the last bin
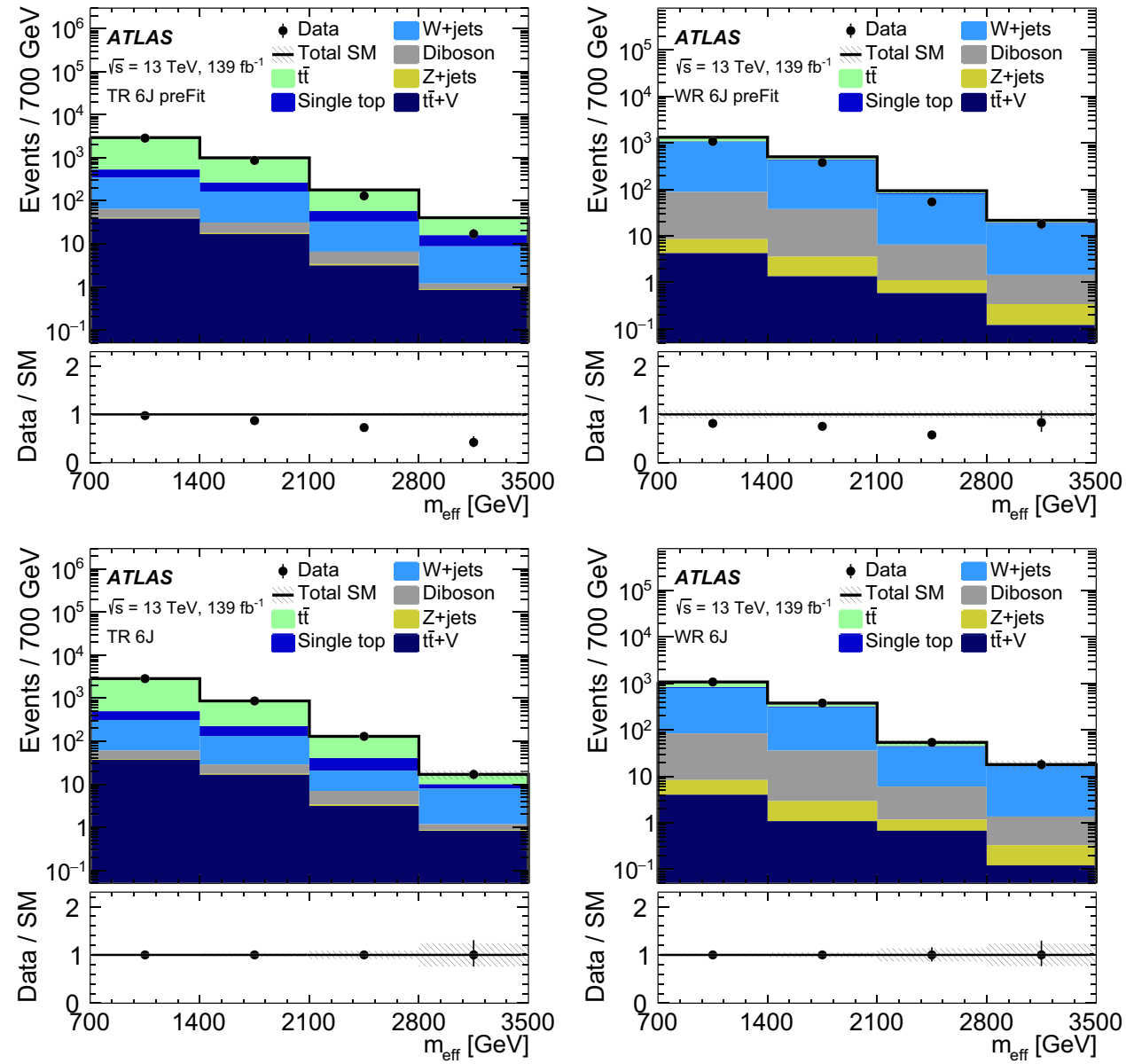

Table 5 Breakdown of the dominant systematic uncertainties in the background estimates in the various signal regions. The numbers are a yield weighted average over the bins. The individual uncertainties can be correlated and do not necessarily add in quadrature to the total background uncertainty. The percentages show the size of the uncertainty relative to the total expected background

\begin{tabular}{|c|c|c|c|c|}
\hline Uncertainty of channel & 2J b-veto & 2J b-tag & $4 \mathrm{~J}$ low-x b-veto & $4 \mathrm{~J}$ low-x b-tag \\
\hline Total background expectation & 346 & 272 & 449 & 810 \\
\hline Total background systematic uncertainty & $8 \%$ & $25 \%$ & $7 \%$ & $13 \%$ \\
\hline Jet energy resolution & $2.5 \%$ & $4 \%$ & $2.9 \%$ & $2.4 \%$ \\
\hline Jet energy scale & $1.8 \%$ & $1.6 \%$ & $2.4 \%$ & $1.5 \%$ \\
\hline$b$-tagging & $1.6 \%$ & $1.7 \%$ & $2.2 \%$ & $1.3 \%$ \\
\hline Lepton uncertainties & $1.0 \%$ & $0.6 \%$ & $0.5 \%$ & $0.34 \%$ \\
\hline$E_{\mathrm{T}}^{\mathrm{miss}} / \mathrm{JVT} /$ pile-up/trigger & $1.9 \%$ & $0.33 \%$ & $0.5 \%$ & $0.6 \%$ \\
\hline$W+$ jets theory uncertainties & $0.8 \%$ & $1.8 \%$ & $1.6 \%$ & $0.9 \%$ \\
\hline$t \bar{t}$ theory uncertainties & $5 \%$ & $23 \%$ & $3.1 \%$ & $12 \%$ \\
\hline Single-top theory uncertainties & $0.7 \%$ & $4 \%$ & $0.9 \%$ & $1.3 \%$ \\
\hline Other theory uncertainties & $2.7 \%$ & $0.8 \%$ & $2.8 \%$ & $0.26 \%$ \\
\hline Normalisation of dominant backgrounds & $1.5 \%$ & $9 \%$ & $1.0 \%$ & $4 \%$ \\
\hline MC statistics & $3.0 \%$ & $1.5 \%$ & $1.6 \%$ & $0.8 \%$ \\
\hline
\end{tabular}


Table 6 Breakdown of the dominant systematic uncertainties in the background estimates in the various signal regions. The numbers are a yield weighted average over the bins. The individual uncertainties can

be correlated and do not necessarily add in quadrature to the total background uncertainty. The percentages show the size of the uncertainty relative to the total expected background

\begin{tabular}{|c|c|c|c|c|}
\hline Uncertainty of channel & 4J high-x b-veto & 4J high-x b-tag & 6J b-veto & 6J b-tag \\
\hline Total background expectation & 117 & 160 & 46 & 196 \\
\hline Total background systematic uncertainty & $25 \%$ & $17 \%$ & $22 \%$ & $15 \%$ \\
\hline Jet energy resolution & $12 \%$ & $2.2 \%$ & $10 \%$ & $2.5 \%$ \\
\hline Jet energy scale & $2.0 \%$ & $1.9 \%$ & $4 \%$ & $1.0 \%$ \\
\hline$b$-tagging & $1.6 \%$ & $1.8 \%$ & $3.4 \%$ & $0.8 \%$ \\
\hline Lepton uncertainties & $4 \%$ & $2.8 \%$ & $1.2 \%$ & $0.7 \%$ \\
\hline$E_{\mathrm{T}}^{\text {miss }} / \mathrm{JVT} /$ pile-up/trigger & $1.2 \%$ & $0.8 \%$ & $0.8 \%$ & $0.8 \%$ \\
\hline$W+$ jets theory uncertainties & $19 \%$ & $7 \%$ & $13 \%$ & $0.5 \%$ \\
\hline$t \bar{t}$ theory uncertainties & $5 \%$ & $14 \%$ & $17 \%$ & $13 \%$ \\
\hline Single-top theory uncertainties & $0.4 \%$ & $3.2 \%$ & $0.9 \%$ & $4 \%$ \\
\hline Other theory uncertainties & $1.8 \%$ & $0.30 \%$ & $2.4 \%$ & $0.32 \%$ \\
\hline Normalisation of dominant backgrounds & $1.8 \%$ & $3.1 \%$ & $2.9 \%$ & $4 \%$ \\
\hline MC statistics & $5 \%$ & $2.2 \%$ & $4 \%$ & $1.4 \%$ \\
\hline
\end{tabular}

of the $t \bar{t}$ uncertainties reported in the tables includes also the statistical component, arising from limited MC statistics, in the uncertainties for all regions. Jet-related uncertainties dominate the detector-related systematic uncertainties.

\section{Results}

The statistical interpretation of the data is based on a profile likelihood method using the HistFitter framework [94,95]. The likelihood function includes a set of Poisson functions representing the yields in each of the control and signal regions. These Poisson functions depend on the observed number of data events in the respective region and the expected numbers of signal and background events. Different parameters are included in the likelihood function to control the normalisation of the backgrounds and the signal or to reflect statistical and systematic uncertainties. The normalisations of the $t \bar{t}$, single-top and $W+$ jets backgrounds are controlled by the respective normalisation factors assigned individually for each $m_{\text {eff }}$ bin in the SRs. This configuration corrects for the mismodelling of the $m_{\mathrm{eff}}$ distribution in the Monte Carlo simulation, as discussed in Sect. 6. An exception is made for the $\mathbf{4 J}$ case in the $m_{\mathrm{eff}}>1600 \mathrm{GeV}$ region, where for each of the $t \bar{t}$ and single-top backgrounds the same normalisation factor is used across the two $m_{\text {eff }}$ bins due to the low statistics in the highest $m_{\text {eff }}$ bin. The yields in the corresponding control regions are sufficient to allow for two different normalisation factors for $W+$ jets, one in the range $m_{\mathrm{eff}} \in[1600,2200] \mathrm{GeV}$ and one for $m_{\mathrm{eff}}>2200 \mathrm{GeV}$. In total, the fit includes nine normalisation factors for $t \bar{t}$ and single top and ten normalisation factors for $W+$ jets. The nor- malisation of the signal is controlled by one common normalisation factor applied to all bins included in the fit. Systematic uncertainties are accommodated through the use of nuisance parameters which are constrained by a Gaussian auxiliary term added to the likelihood.

In a background-only fit, only the control regions are used to constrain the likelihood. A signal contribution is neglected in the fit, so the signal normalisation parameter is dropped. The observed yields in the VRs are found to be compatible with the background expectation obtained from this fit, with the largest deviation of data from MC over the 18 bins having a statistical significance of about $2 \sigma$. Background predictions in the signal regions are compared with the observed data in Tables 7, 8, 9, 10 and illustrated in Figures 5, 6, 7. No significant excess of events is observed.

Using the discovery signal regions defined in Table 2, a test is performed for the presence of beyond-the-SM physics in a model-independent fit in each signal region. The signal contribution is only considered in the respective signal region, and not in the CRs, and therefore a conservative background estimate is obtained in the signal regions. Table 11 shows the observed and expected upper limits $\left(S_{\mathrm{obs}}^{95}\right.$ and $S_{\mathrm{exp}}^{95}$, respectively) on the number of signal events, at $95 \%$ confidence level (CL) using the $\mathrm{CL}_{\mathrm{s}}$ prescription [96]. Also reported is the visible cross-section upper limit $\left(\langle\epsilon \sigma\rangle_{\mathrm{obs}}^{95}\right)$, which is the upper limit on the cross-section times the reconstruction efficiency and region acceptance. The table also presents the discovery $p$-values $\left(p_{0}\right)$, which quantify the probability to observe at least as many events as expected in the background-only assumption, the $\mathrm{CL}_{\mathrm{b}}$ value, i.e. the confidence level observed for the background-only hypothesis, and the associated significance. 
Table 7 Observed event yields and the background expectation obtained by a background fit in the 2J SRs with an integrated luminosity of $139 \mathrm{fb}^{-1}$. Each column corresponds to a bin in $m_{\text {eff }}$. Uncertainties reported for the fitted background estimates combine statistical (in the simulated event yields) and systematic uncertainties. The uncertainties in this table are symmetrised for error propagation purposes
Table 8 Observed event yields and the background expectation obtained by a background fit in the 4J high-x SRs with an integrated luminosity of $139 \mathrm{fb}^{-1}$. Each column corresponds to a bin in $m_{\text {eff }}$. Uncertainties reported for the fitted background estimates combine statistical (in the simulated event yields) and systematic uncertainties. The uncertainties in this table are symmetrised for error propagation purposes but are truncated at zero to remain within the physical boundaries

\begin{tabular}{llll}
\hline 2J b-veto & Bin 1 & Bin 2 & Bin 3 \\
& {$[700,1300] \mathrm{GeV}$} & {$[1300,1900] \mathrm{GeV}$} & $>1900 \mathrm{GeV}$ \\
\hline Observed events & 280 & 84 & 22 \\
Total SM background events & $261 \pm 22$ & $73 \pm 12$ & $12.8 \pm 2.2$ \\
$t \bar{t}$ events & $19 \pm 13$ & $11 \pm 7$ & $1.3 \pm 0.6$ \\
$W+$ jets events & $155 \pm 14$ & $28 \pm 5$ & $3.4 \pm 1.5$ \\
$Z+$ jets events & $14 \pm 5$ & $4.3 \pm 0.6$ & $1.37 \pm 0.19$ \\
Single-top events & $5 \pm 4$ & $2.9 \pm 2.3$ & $1.1 \pm 0.9$ \\
Diboson events & $66 \pm 8$ & $26.0 \pm 3.4$ & $5.5 \pm 0.7$ \\
$t \bar{t}+V$ events & $1.32 \pm 0.16$ & $0.47 \pm 0.23$ & $0.041 \pm 0.023$ \\
\hline 2J b-tag & Bin 1 & Bin 2 & Bin 3 \\
& {$[700,1300] \mathrm{GeV}$} & {$[1300,1900] \mathrm{GeV}$} & $>1900 \mathrm{GeV}$ \\
\hline Observed events & 154 & 106 & 21 \\
Total SM background & $134 \pm 36$ & $123 \pm 33$ & $16 \pm 6$ \\
$t \bar{t}$ events & $74 \pm 35$ & $90 \pm 32$ & $10 \pm 5$ \\
$W+$ jets events & $20 \pm 6$ & $6.3 \pm 2.1$ & $0.7 \pm 0.5$ \\
$Z+$ jets events & $5.0 \pm 0.7$ & $2.0 \pm 2.0$ & $0.55 \pm 0.10$ \\
Single-top events & $18 \pm 7$ & $15 \pm 6$ & $2.6 \pm 1.6$ \\
Diboson events & $9.0 \pm 1.4$ & $4.3 \pm 1.5$ & $1.04 \pm 0.17$ \\
$t \bar{t}+V$ events & $8.4 \pm 0.7$ & $5.0 \pm 0.5$ & $0.63 \pm 0.09$ \\
\hline
\end{tabular}

\begin{tabular}{llll}
\hline 4J high-x b-veto & Bin 1 & Bin 2 & Bin 3 \\
& {$[1000,1600] \mathrm{GeV}$} & {$[1600,2200] \mathrm{GeV}$} & $>2200 \mathrm{GeV}$ \\
\hline Observed events & 104 & 32 & 11 \\
Total SM background & $92 \pm 32$ & $18 \pm 4$ & $7.1 \pm 2.3$ \\
$t \bar{t}$ events & $9 \pm 5$ & $1.2 \pm 0.4$ & $0.32 \pm 0.11$ \\
$W+$ jets events & $61 \pm 30$ & $9 \pm 4$ & $3.6 \pm 1.7$ \\
$Z+$ jets events & $1.5 \pm 0.6$ & $0.8 \pm 0.4$ & $0.26 \pm 0.14$ \\
Single-top events & $0.3_{-0.3}^{+0.5}$ & $0.006_{-0.006}^{+0.026}$ & $1.3 \pm 0.8$ \\
Diboson events & $18.7 \pm 2.9$ & $6.1 \pm 0.9$ & $1.59 \pm 0.29$ \\
$t \bar{t}+V$ events & $0.65 \pm 0.15$ & $0.09_{-0.09}^{+0.13}$ & $0.029 \pm 0.023$ \\
\hline 4J high-x b-tag & $\mathrm{Bin} 1$ & $\mathrm{Bin} 2$ & $\mathrm{Bin} 3$ \\
& {$[1000,1600] \mathrm{GeV}$} & {$[1600,2200] \mathrm{GeV}$} & $>2200 \mathrm{GeV}$ \\
\hline Observed events & 121 & 26 & 8 \\
Total SM background & $127 \pm 27$ & $25 \pm 5$ & $7.7 \pm 2.1$ \\
$t \bar{t}$ events & $90 \pm 24$ & $13.1 \pm 2.8$ & $2.0 \pm 0.5$ \\
$W+$ jets events & $18 \pm 9$ & $4.6 \pm 2.4$ & $1.1 \pm 0.4$ \\
$Z+$ jets events & $0.32 \pm 0.10$ & $0.01_{-0.01}^{+0.13}$ & $0.15 \pm 0.09$ \\
Single-top events & $10 \pm 4$ & $4.9 \pm 1.8$ & $3.6 \pm 1.7$ \\
Diboson events & $3.1 \pm 0.6$ & $1.20 \pm 0.34$ & $0.41 \pm 0.15$ \\
$t \bar{t}+V$ events & $5.8 \pm 0.5$ & $1.51 \pm 0.17$ & $0.39 \pm 0.08$ \\
\hline
\end{tabular}


Table 9 Observed event yields and the background expectation obtained by a background fit in the $\mathbf{4 J}$ low-x SRs with an integrated luminosity of $139 \mathrm{fb}^{-1}$. Each column corresponds to a bin in $m_{\text {eff }}$. Uncertainties reported for the fitted background estimates combine statistical (in the simulated event yields) and systematic uncertainties. The uncertainties in this table are symmetrised for error propagation purposes but are truncated at zero to remain within the physical boundaries

\begin{tabular}{llll}
\hline 4J low-x b-veto & Bin 1 & Bin 2 & Bin 3 \\
& {$[1000,1600] \mathrm{GeV}$} & {$[1600,2200] \mathrm{GeV}$} & $>2200 \mathrm{GeV}$ \\
\hline Observed events & 393 & 57 & 10 \\
Total SM background & $383 \pm 27$ & $56 \pm 6$ & $9.5 \pm 1.7$ \\
$t \bar{t}$ events & $72 \pm 15$ & $8.7 \pm 1.8$ & $1.56 \pm 0.35$ \\
$W+$ jets events & $179 \pm 23$ & $23 \pm 4$ & $3.4 \pm 1.4$ \\
$Z+$ jets events & $4.7 \pm 0.9$ & $0.73 \pm 0.19$ & $0.16 \pm 0.04$ \\
Single-top events & $12 \pm 5$ & $3.3 \pm 1.3$ & $0.16_{-0.16}^{+0.25}$ \\
Diboson events & $110 \pm 15$ & $20.5 \pm 2.8$ & $4.2 \pm 0.6$ \\
$t \bar{t}+V$ events & $5.6 \pm 0.6$ & $0.54 \pm 0.22$ & $0.070 \pm 0.031$ \\
\hline 4J low-x b-tag & Bin 1 & Bin 2 & Bin 3 \\
& {$[1000,1600] \mathrm{GeV}$} & {$[1600,2200] \mathrm{GeV}$} & $>2200 \mathrm{GeV}$ \\
\hline Observed events & 695 & 79 & 11 \\
Total SM background & $701 \pm 90$ & $94 \pm 19$ & $15 \pm 4$ \\
$t \bar{t}$ events & $510 \pm 90$ & $60 \pm 18$ & $9.0 \pm 2.9$ \\
$W+$ jets events & $36 \pm 9$ & $7.0 \pm 1.7$ & $0.96 \pm 0.35$ \\
$Z+$ jets events & $1.7 \pm 0.6$ & $0.36 \pm 0.08$ & $0.035 \pm 0.020$ \\
Single-top events & $88 \pm 12$ & $19 \pm 6$ & $3.9 \pm 2.5$ \\
Diboson events & $21.1 \pm 3.2$ & $4.3 \pm 0.6$ & $0.90 \pm 0.13$ \\
$t \bar{t}+V$ events & $41.5 \pm 3.0$ & $3.9 \pm 0.6$ & $0.45 \pm 0.10$ \\
\hline
\end{tabular}

Table 10 Observed event yields and the background expectation obtained by a background fit in the 6J SRs with an integrated luminosity of $139 \mathrm{fb}^{-1}$. Each column corresponds to a bin in $m_{\mathrm{eff}}$. Uncertainties reported for the fitted background estimates combine statistical (in the simulated event yields) and systematic uncertainties. The uncertainties in this table are symmetrised for error propagation purposes but are truncated at zero to remain within the physical boundaries

\begin{tabular}{|c|c|c|c|c|}
\hline 6J b-veto & $\begin{array}{l}\text { Bin } 1 \\
{[700,1400] \mathrm{GeV}}\end{array}$ & $\begin{array}{l}\text { Bin } 2 \\
{[1400,2100] \mathrm{GeV}}\end{array}$ & $\begin{array}{l}\text { Bin } 3 \\
{[2100,2800] \mathrm{GeV}}\end{array}$ & $\begin{array}{l}\text { Bin } 4 \\
>2800 \mathrm{GeV}\end{array}$ \\
\hline Observed events & 19 & 16 & 3 & 2 \\
\hline Total SM background & $25 \pm 8$ & $15.9 \pm 2.5$ & $3.5 \pm 0.5$ & $1.8 \pm 0.6$ \\
\hline$t \bar{t}$ events & $10 \pm 6$ & $4.6 \pm 1.7$ & $0.77 \pm 0.26$ & $0.09 \pm 0.07$ \\
\hline$W+$ jets events & $7 \pm 5$ & $5.2 \pm 1.5$ & $1.2 \pm 0.5$ & $0.6 \pm 0.4$ \\
\hline$Z+$ jets events & $0.23_{-0.23}^{+0.23}$ & $0.25 \pm 0.07$ & $0.12 \pm 0.05$ & $0.060 \pm 0.025$ \\
\hline Single-top events & $0.5_{-0.5}^{+0.8}$ & $0.3_{-0.3}^{+0.5}$ & $0.0 \pm 0.0$ & $0.5 \pm 0.4$ \\
\hline Diboson events & $6.2 \pm 1.4$ & $5.2 \pm 0.9$ & $1.31 \pm 0.26$ & $0.52 \pm 0.16$ \\
\hline$t \bar{t}+V$ events & $0.90 \pm 0.18$ & $0.47 \pm 0.11$ & $0.06 \pm 0.04$ & $0.012_{-0.012}^{+0.021}$ \\
\hline 6J b-tag & $\begin{array}{l}\text { Bin } 1 \\
{[700,1400] \mathrm{GeV}}\end{array}$ & $\begin{array}{l}\operatorname{Bin} 2 \\
{[1400,2100] \mathrm{GeV}}\end{array}$ & $\begin{array}{l}\operatorname{Bin} 3 \\
{[2100,2800] \mathrm{GeV}}\end{array}$ & $\begin{array}{l}\text { Bin } 4 \\
>2800 \mathrm{GeV}\end{array}$ \\
\hline Observed events & 117 & 68 & 7 & 2 \\
\hline Total SM background & $110 \pm 17$ & $70 \pm 11$ & $13.6 \pm 3.1$ & $2.4 \pm 1.0$ \\
\hline$t \bar{t}$ events & $90 \pm 17$ & $52 \pm 10$ & $10.2 \pm 2.8$ & $0.9 \pm 0.6$ \\
\hline$W+$ jets events & $2.0 \pm 1.3$ & $1.6 \pm 0.8$ & $0.53 \pm 0.16$ & $0.46 \pm 0.19$ \\
\hline$Z+$ jets events & $0.05_{-0.05}^{+0.09}$ & $0.12 \pm 0.05$ & $0.06 \pm 0.04$ & $0.08 \pm 0.04$ \\
\hline Single-top events & $4.6 \pm 3.1$ & $9 \pm 5$ & $1.3 \pm 1.1$ & $0.6_{-0.6}^{+0.8}$ \\
\hline Diboson events & $1.62 \pm 0.32$ & $1.64 \pm 0.31$ & $0.57 \pm 0.13$ & $0.14 \pm 0.07$ \\
\hline$t \bar{t}+V$ events & $11.5 \pm 1.5$ & $5.1 \pm 0.8$ & $0.95 \pm 0.25$ & $0.20 \pm 0.13$ \\
\hline
\end{tabular}


Fig. 5 Comparison of the observed and expected event yields in all signal regions in the background-only fit

Fig. 6 Post-fit $m_{\text {eff }}$

distributions in the exclusion $\mathbf{2 J}$ and $\mathbf{4 J}$ high-x signal regions.

The uncertainty bands include all statistical and systematic uncertainties. The dashed lines represent benchmark signal points. Overflow events are included in the last bin
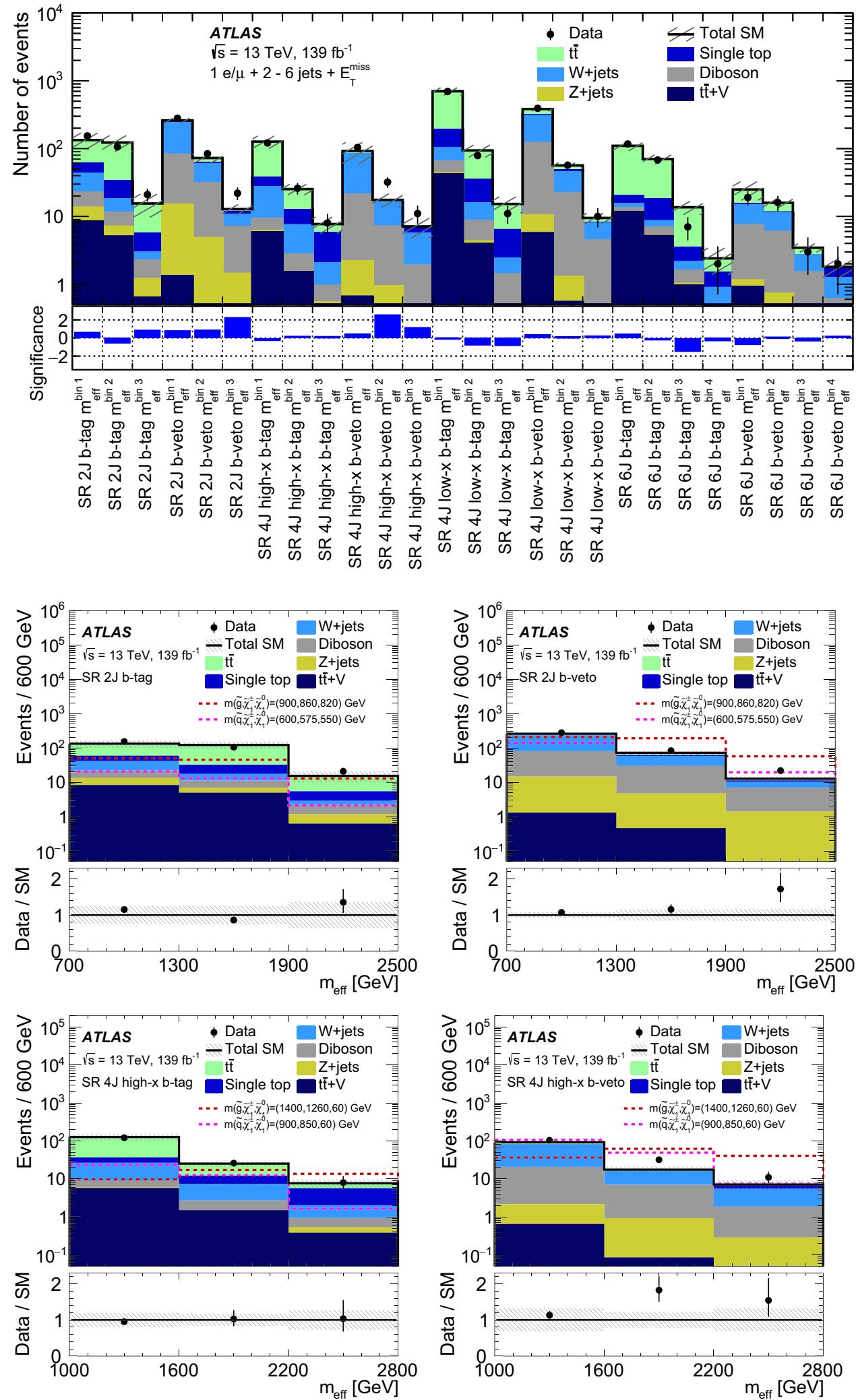
Fig. 7 Post-fit $m_{\text {eff }}$ distributions in the exclusion $\mathbf{4 J}$ low-x and $\mathbf{6 J}$ signal regions. The uncertainty bands include all statistical and systematic uncertainties. The dashed lines represent benchmark signal points. Overflow events are included in the last bin
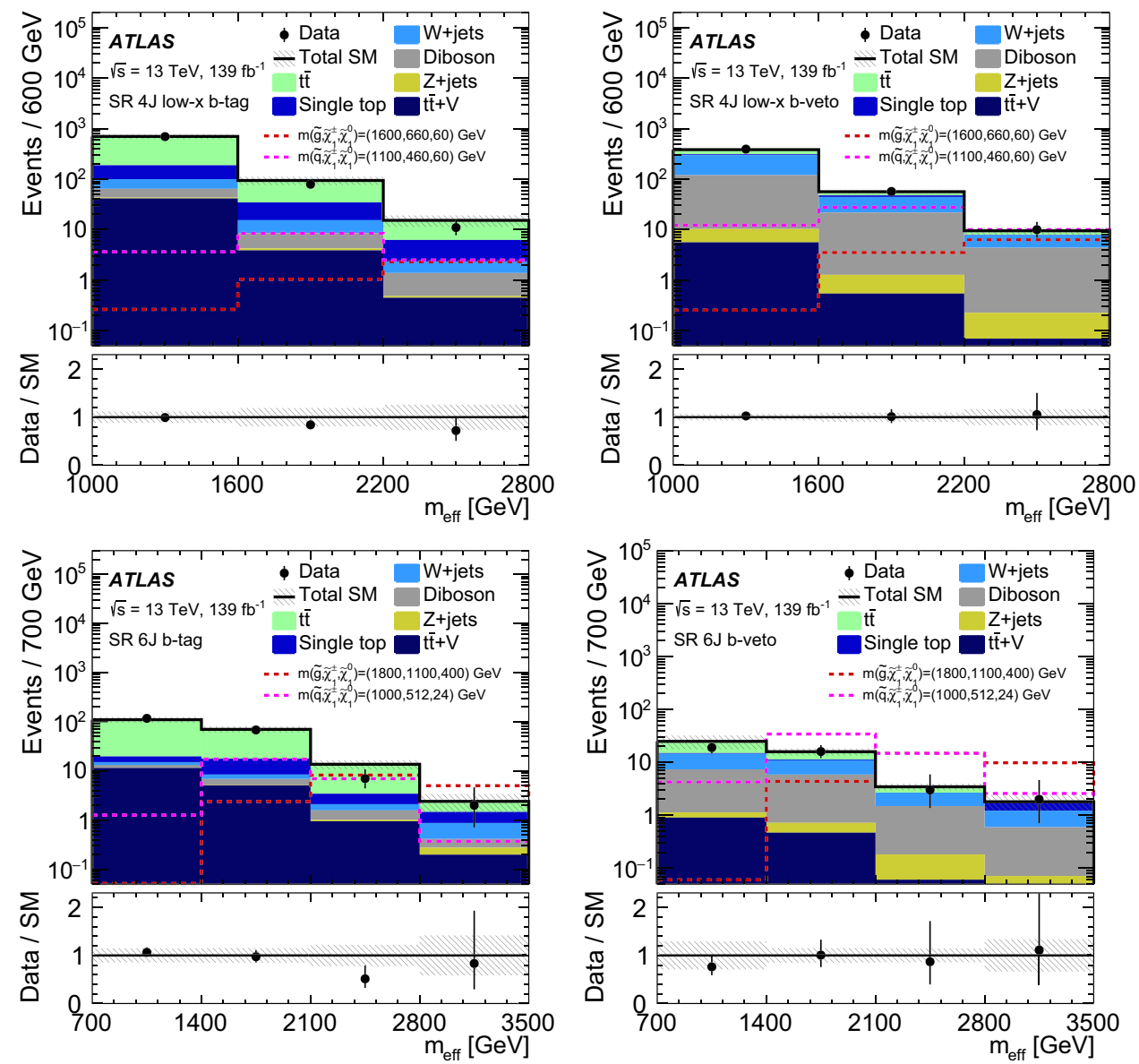

Table 11 Results of the model-independent limit fits. For each SR defined in Table 2, the observed events and the total SM background, and the observed $95 \%$ CL upper limits on the visible cross-section $\langle\epsilon \sigma\rangle_{\mathrm{obs}}^{95}$ and on the number of signal events $S_{\mathrm{obs}}^{95}$ are given. The sixth column, $S_{\text {exp }}^{95}$, shows the $95 \%$ CL upper limit on the number of signal events, given the expected number (and $\pm 1 \sigma$ excursions of the expectation) of background events. The last two columns indicate the $\mathrm{CL}_{b}$ value, i.e. the confidence level observed for the background-only hypothesis, the discovery $p$-value $p(s=0)$ and the significance $Z$. In case of fewer events than the fitted background estimate observed, the p-values are capped at 0.5

\begin{tabular}{llllllll}
\hline SR $_{\text {disc }}$ & Observed events & Total SM background & $\langle\epsilon \sigma\rangle_{\text {obs }}^{95}[\mathrm{fb}]$ & $S_{\text {obs }}^{95}$ & $S_{\text {exp }}^{95}$ & $\mathrm{CL}_{\mathrm{b}}$ & $p(s=0)(Z)$ \\
\hline 2J (gluino) & 22 & $12.8 \pm 2.2$ & 0.14 & 19.0 & $10.1_{-2.3}^{+4.0}$ & 0.98 & $0.02(1.97)$ \\
$\mathbf{2 J}$ (squark) & 106 & $86 \pm 12$ & 0.34 & 47.8 & $31.3_{-9.6}^{+13.0}$ & 0.90 & $0.09(1.33)$ \\
4J high-x & 11 & $7.1 \pm 2.3$ & 0.09 & 12.0 & $8.3_{-1.5}^{+3.5}$ & 0.87 & $0.13(1.12)$ \\
4J low-x & 10 & $9.5 \pm 1.7$ & 0.06 & 8.9 & $8.4_{-2.0}^{+3.3}$ & 0.57 & $0.42(0.19)$ \\
6J (gluino) & 2 & $1.8 \pm 0.6$ & 0.03 & 4.7 & $4.3_{-0.8}^{+1.9}$ & 0.59 & $0.41(0.24)$ \\
6J (squark) & 5 & $5.3 \pm 0.8$ & 0.04 & 6.0 & $6.0_{-1.5}^{+24.0}$ & 0.48 & $0.50(0)$ \\
\hline
\end{tabular}

Observed and expected exclusion limits at 95\% CL are calculated for the gluino and squark one-step models using all statistically independent binned signal and control regions in a model-dependent fit. For this exclusion fit, the signal contribution, adjusted using a single floating normalisation factor, is considered in all control and signal regions. The background normalisation factors are simultaneously deter- mined in the same fit. Specific sparticle masses in the gluino or squark one-step models can be excluded if the upper limit of the signal normalisation factor is less than unity.

Figure 8 shows the expected and observed exclusion limits. Gluino masses up to 2.2 and 2.05 can be excluded for $\tilde{\chi}_{1}^{0}$ masses less than $400 \mathrm{GeV}$ and 1 respectively, while squark masses up to 1.37 can be excluded for low-mass $\tilde{\chi}_{1}^{0}$. Ben- 

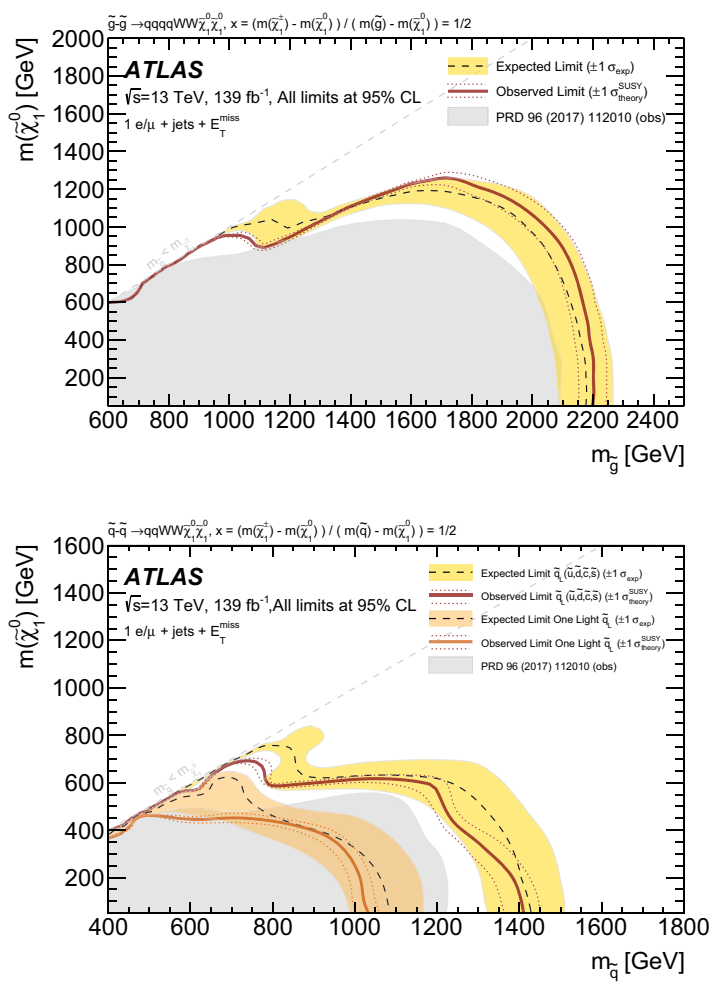

Fig. 8 Exclusion limits for the gluino one-step $x=1 / 2$ (top left), gluino one-step variable- $x$ (top right), squark one-step $x=1 / 2$ (bottom left) and squark one-step variable- $x$ (bottom right) scenarios. The red solid line corresponds to the observed limit, with the red dotted lines indicating the $\pm 1 \sigma$ variation of the limit due to the effect of theoretical scale and PDF uncertainties in the signal cross-section, for scenarios where the four left-handed squarks of the first two generations $\left(\tilde{u}_{\mathrm{L}}, \tilde{d}_{\mathrm{L}}, \tilde{c}_{\mathrm{L}}, \tilde{s}_{\mathrm{L}}\right)$ are mass degenerate. The dark grey dashed line indi-

efiting from the increased integrated luminosity, the current observed limit exceeds the previous ATLAS limit by about $100 \mathrm{GeV}$ in $m_{\tilde{g}}$ and in $m_{\tilde{q}}$ for low-mass $\tilde{\chi}_{1}^{0}$. In squark one-step models in which only a single squark flavour is kinematically accessible, squark masses up to about 1.0 can be excluded.

\section{Conclusion}

A search for gluinos and squarks in events with one isolated lepton, jets and missing transverse momentum is presented. The analysis uses $139 \mathrm{fb}^{-1}$ of proton-proton collision data at a centre-of-mass energy of 13 collected by the ATLAS experiment at the LHC. Four signal regions requiring from at least two to at least six jets are used to cover a broad spectrum of the targeted SUSY model parameter space. Three signal regions defined using high- $p_{\mathrm{T}}$ lepton selections target models with large mass differences between the supersymmetric particles. A separate, low- $p_{\mathrm{T}}$ lepton region is designed to enhance the sensitivity to models with compressed mass spectra. The data
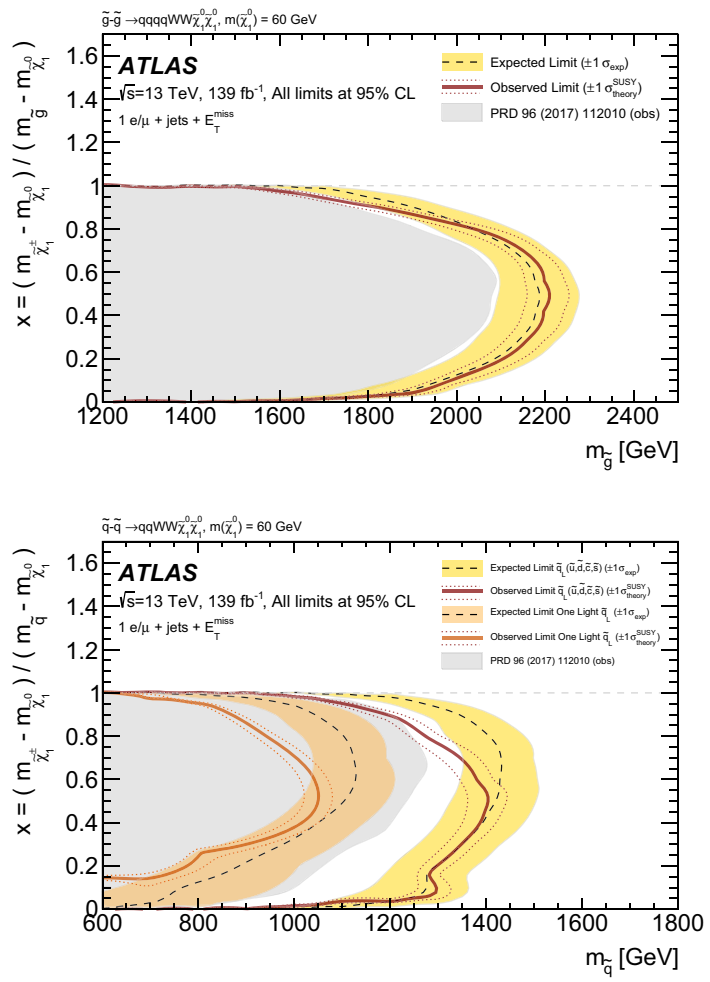

cates the expected limit with the yellow band representing the impact of the $\pm 1 \sigma$ variation of the median expected limit due to the experimental and theoretical uncertainties. The orange solid and the dashed lines show the squark one-step $x=1 / 2$ (left) and squark one-step variable$x$ (right) scenarios for cases in which only a single squark flavour is kinematically accessible. For reference, exclusion bounds from previous searches with $36.1 \mathrm{fb}^{-1}$ of data at 13 centre-of-mass energy [23] are overlaid as the grey area

agree with the Standard Model background prediction in the signal regions. For all signal regions, limits on the visible cross-section are derived in models of new physics within the kinematic requirements of this search. In addition, exclusion limits are placed on models with gluino/squark production and subsequent decays via an intermediate chargino to the lightest neutralino. This search extends the exclusion limit by $100 \mathrm{GeV}$ (gluino) and $180 \mathrm{GeV}$ (squark) for a massless LSP with respect to the previous search [23] owing to a more solid background estimation technique and an increased statistical sample. Gluino (Squark) masses up to around 2.2 (1.4) are excluded for a $\tilde{\chi}_{1}^{0}$ mass lower than $200 \mathrm{GeV}$, while for scenarios with a single accessible squark flavour, squark masses up to around 1.04 are excluded.

Acknowledgements We thank CERN for the very successful operation of the LHC, as well as the support staff from our institutions without whom ATLAS could not be operated efficiently. We acknowledge the support of ANPCyT, Argentina; YerPhI, Armenia; ARC, Australia; BMWFW and FWF, Austria; ANAS, Azerbaijan; SSTC, Belarus; CNPq and FAPESP, Brazil; NSERC, NRC and CFI, Canada; CERN; ANID, Chile; CAS, MOST and NSFC, China; COLCIEN- 
CIAS, Colombia; MSMT CR, MPO CR and VSC CR, Czech Republic; DNRF and DNSRC, Denmark; IN2P3-CNRS and CEA-DRF/IRFU, France; SRNSFG, Georgia; BMBF, HGF and MPG, Germany; GSRT, Greece; RGC and Hong Kong SAR, China; ISF and Benoziyo Center, Israel; INFN, Italy; MEXT and JSPS, Japan; CNRST, Morocco; NWO, Netherlands; RCN, Norway; MNiSW and NCN, Poland; FCT, Portugal; MNE/IFA, Romania; JINR; MES of Russia and NRC KI, Russian Federation; MESTD, Serbia; MSSR, Slovakia; ARRS and MIZŠ, Slovenia; DST/NRF, South Africa; MICINN, Spain; SRC and Wallenberg Foundation, Sweden; SERI, SNSF and Cantons of Bern and Geneva, Switzerland; MOST, Taiwan; TAEK, Turkey; STFC, United Kingdom; DOE and NSF, United States of America. In addition, individual groups and members have received support from BCKDF, CANARIE, Compute Canada, CRC and IVADO, Canada; Beijing Municipal Science \& Technology Commission, China; COST, ERC, ERDF, Horizon 2020 and Marie Skłodowska-Curie Actions, European Union; Investissements d'Avenir Labex, Investissements d'Avenir Idex and ANR, France; DFG and AvH Foundation, Germany; Herakleitos, Thales and Aristeia programmes co-financed by EU-ESF and the Greek NSRF, Greece; BSFNSF and GIF, Israel; La Caixa Banking Foundation, CERCA Programme Generalitat de Catalunya and PROMETEO and GenT Programmes Generalitat Valenciana, Spain; Göran Gustafssons Stiftelse, Sweden; The Royal Society and Leverhulme Trust, United Kingdom. The crucial computing support from all WLCG partners is acknowledged gratefully, in particular from CERN, the ATLAS Tier-1 facilities at TRIUMF (Canada), NDGF (Denmark, Norway, Sweden), CCIN2P3 (France), KIT/GridKA (Germany), INFN-CNAF (Italy), NLT1 (Netherlands), PIC (Spain), ASGC (Taiwan), RAL (UK) and BNL (USA), the Tier-2 facilities worldwide and large non-WLCG resource providers. Major contributors of computing resources are listed in Ref. [97].

Data Availability Statement This manuscript has no associated data or the data will not be deposited. [Authors' comment: All ATLAS scientific output is published in journals, and preliminary results are made available in Conference Notes. All are openly available, without restriction on use by external parties beyond copyright law and the standard conditions agreed by CERN. Data associated with journal publications are also made available: tables and data from plots (e.g. cross section values, likelihood profiles, selection efficiencies, cross section limits, ...) are stored in appropriate repositories such as HEPDATA (http:// hepdata.cedar.ac.uk/). ATLAS also strives to make additional material related to the paper available that allows a reinterpretation of the data in the context of new theoretical models. For example, an extended encapsulation of the analysis is often provided for measurements in the framework of RIVET (http://rivet.hepforge.org/)." This information is taken from the ATLAS Data Access Policy, which is a public document that can be downloaded from http://opendata.cern.ch/record/413 [opendata.cern.ch].]

Open Access This article is licensed under a Creative Commons Attribution 4.0 International License, which permits use, sharing, adaptation, distribution and reproduction in any medium or format, as long as you give appropriate credit to the original author(s) and the source, provide a link to the Creative Commons licence, and indicate if changes were made. The images or other third party material in this article are included in the article's Creative Commons licence, unless indicated otherwise in a credit line to the material. If material is not included in the article's Creative Commons licence and your intended use is not permitted by statutory regulation or exceeds the permitted use, you will need to obtain permission directly from the copyright holder. To view a copy of this licence, visit http://creativecomm ons.org/licenses/by/4.0/.

Funded by SCOAP ${ }^{3}$.

\section{References}

1. ATLAS Collaboration, Observation of a new particle in the search for the Standard Model Higgs boson with the ATLAS detector at the LHC. Phys. Lett. B 716, 1 (2012). arXiv:1207.7214 [hep-ex]

2. C.M.S. Collaboration, Observation of a new boson at a mass of 125 $\mathrm{GeV}$ with the CMS experiment at the LHC. Phys. Lett. B 716, 30 (2012). arXiv:1207.7235 [hep-ex]

3. ATLAS and CMS Collaborations, Combined measurement of the Higgs boson mass in pp collisions at $\mathrm{v} \mathrm{s}=7$ and $8 \mathrm{TeV}$ with the ATLAS and CMS Experiments. Phys. Rev. Lett. 114, 191803 (2015). arXiv:1503.07589 [hep-ex]

4. ATLAS and CMS Collaborations, Measurements of the Higgs boson production and decay rates and constraints on its couplings from a combined ATLAS and CMS analysis of the LHC pp collision data at $\mathrm{v}$ s $=7$ and $8 \mathrm{TeV}$. JHEP 08, 045 (2016). arXiv: 1606.02266 [hep-ex]

5. N. Sakai, Naturalness in supersymmetric GUTS. Z. Phys. C 11, $153(1981)$

6. S. Dimopoulos, S. Raby, F. Wilczek, Supersymmetry and the scale of unification. Phys. Rev. D 24, 1681 (1981)

7. L.E. Ibanez, G.G. Ross, Low-energy predictions in supersymmetric grand unified theories. Phys. Lett. B 105, 439 (1981)

8. S. Dimopoulos, H. Georgi, Softly broken supersymmetry and SU(5). Nucl. Phys. B 193, 150 (1981)

9. Y.A. Golfand, E.P. Likhtman, Extension of the algebra of Poincare group generators and violation of $P$ invariance. JETP Lett. 13, 323 (1971). [Pisma Zh. Eksp. Teor. Fiz. 13, 452 (1971)]

10. D.V. Volkov, V.P. Akulov, Is the neutrino a goldstone particle? Phys. Lett. B 46, 109 (1973)

11. J. Wess, B. Zumino, Supergauge transformations in fourdimensions. Nucl. Phys. B 70, 39 (1974)

12. J. Wess, B. Zumino, Supergauge invariant extension of quantum electrodynamics. Nucl. Phys. B 78, 1 (1974)

13. S. Ferrara, B. Zumino, Supergauge invariant Yang-Mills theories. Nucl. Phys. B 79, 413 (1974)

14. A. Salam, J.A. Strathdee, Super-symmetry and non-Abelian gauges. Phys. Lett. B 51, 353 (1974)

15. G.R. Farrar, P. Fayet, Phenomenology of the production, decay, and detection of new hadronic states associated with supersymmetry. Phys. Lett. B 76, 575 (1978)

16. H. Goldberg, Constraint on the photino mass from cosmology. Phys. Rev. Lett. 50, 1419 (1983). [Erratum: Phys. Rev. Lett. 103, 099905 (2009)]

17. J.R. Ellis, J.S. Hagelin, D.V. Nanopoulos, K.A. Olive, M. Srednicki, Supersymmetric relics from the big bang. Nucl. Phys. B 238, 453 (1984)

18. P. Fayet, Supersymmetry and weak, electromagnetic and strong interactions. Phys. Lett. B 64, 159 (1976)

19. P. Fayet, Spontaneously broken supersymmetric theories of weak, electromagnetic and strong interactions. Phys. Lett. B 69, 489 (1977)

20. J. Alwall, P. Schuster, N. Toro, Simplified models for a first characterization of new physics at the LHC. Phys. Rev. D 79, 075020 (2009). arXiv:0810.3921 [hep-ph]

21. D. Alves et al., Simplified models for LHC new physics searches. J. Phys. G 39, 105005 (2012). arXiv:1105.2838 [hep-ph]

22. ATLAS Collaboration, Further search for supersymmetry at $\mathrm{v} s=$ $7 \mathrm{TeV}$ in final states with jets, missing transverse momentum and isolated leptons with the ATLAS detector. Phys. Rev. D 86, 092002 (2012). arXiv:1208.4688 [hep-ex]

23. ATLAS Collaboration, Search for squarks and gluinos in events with an isolated lepton, jets, and missing transverse momentum at $\mathrm{v} \mathrm{s}=13 \mathrm{TeV}$ with the ATLAS detector. Phys. Rev. D 96, 112010 (2017). arXiv:1708.08232 [hep-ex] 
24. CMS Collaboration, Search for supersymmetry in events with one lepton and multiple jets in proton-proton collisions at $\mathrm{v} \mathrm{s}=13 \mathrm{TeV}$. Phys. Rev. D 95, 012011 (2017). arXiv:1609.09386 [hep-ex]

25. CMS Collaboration, Search for supersymmetry in multijet events with missing transverse momentum in proton-proton collisions at 13 TeV. Phys. Rev. D 96, 032003 (2017). arXiv:1704.07781 [hepex]

26. ATLAS Collaboration, The ATLAS experiment at the CERN large hadron collider. JINST 3, S08003 (2008)

27. ATLAS Collaboration, ATLAS Insertable B-Layer Technical Design Report, ATLAS-TDR-19 (2010). https://cds.cern.ch/ record/1291633. ATLAS Insertable B-Layer Technical Design Report Addendum, ATLAS-TDR-19-ADD-1 (2012). https://cds. cern.ch/record/1451888

28. B. Abbott et al., Production and integration of the ATLAS Insertable B-Layer. JINST 13, T05008 (2018). arXiv:1803.00844 [physics.ins-det]

29. ATLAS Collaboration, Performance of the ATLAS trigger system in 2015. Eur. Phys. J. C 77, 317 (2017). arXiv:1611.09661 [hep-ex]

30. ATLAS Collaboration, Luminosity determination in pp collisions at $\mathrm{v} \mathrm{s}=13 \mathrm{TeV}$ using the ATLAS detector at the LHC, ATLASCONF-2019-021 (2019). https://cds.cern.ch/record/2677054

31. G. Avoni et al., The new LUCID-2 detector for luminosity measurement and monitoring in ATLAS. JINST 13, P07017 (2018)

32. ATLAS Collaboration, The simulation principle and performance of the ATLAS fast calorimeter simulation FastCaloSim, ATLPHYS-PUB-2010-013 (2010). https://cds.cern.ch/record/1300517

33. S. Agostinelli et al., GEANT4—a simulation toolkit. Nucl. Instrum. Methods A 506, 250 (2003)

34. ATLAS Collaboration, The ATLAS simulation infrastructure. Eur. Phys. J. C 70, 823 (2010). arXiv:1005.4568 [physics.ins-det]

35. T. Sjöstrand, S. Mrenna, P.Z. Skands, A brief introduction to PYTHIA 8.1. Comput. Phys. Commun. 178, 852 (2008). arXiv:0710.3820 [hep-ph]

36. R.D. Ball et al., Parton distributions with LHC data. Nucl. Phys. B 867, 244 (2013). arXiv:1207.1303 [hep-ph]

37. ATLAS Collaboration, The Pythia 8 A3 tune description of ATLAS minimum bias and inelastic measurements incorporating the Donnachie-Landshoff diffractive model, ATL-PHYS-PUB2016-017 (2016). https://cds.cern.ch/record/2206965

38. J. Alwall et al., The automated computation of tree-level and nextto-leading order differential cross sections, and their matching to parton shower simulations. JHEP 07, 079 (2014). arXiv:1405.0301 [hep-ph]

39. W. Beenakker, C. Borschensky, M. Krämer, A. Kulesza, E. Laenen, NNLL-fast: predictions for coloured supersymmetric particle production at the LHC with threshold and Coulomb resummation. JHEP 12, 133 (2016). arXiv:1607.07741 [hep-ph]

40. W. Beenakker et al., NNLL resummation for squark-antisquark and gluino-pair production at the LHC. JHEP 12, 023 (2014). arXiv:1404.3134 [hep-ph]

41. W. Beenakker et al., Towards NNLL resummation: hard matching coefficients for squark and gluino hadroproduction. JHEP 10, 120 (2013). arXiv:1304.6354 [hep-ph]

42. W. Beenakker et al., NNLL resummation for squark-antisquark pair production at the LHC. JHEP 01, 076 (2012). arXiv: 1110.2446 [hep-ph]

43. W. Beenakker et al., Soft-gluon resummation for squark and gluino hadroproduction. JHEP 12, 041 (2009). arXiv:0909.4418 [hep-ph]

44. A. Kulesza, L. Motyka, Soft gluon resummation for the production of gluino-gluino and squark-antisquark pairs at the LHC. Phys. Rev. D 80, 095004 (2009). arXiv:0905.4749 [hep-ph]

45. A. Kulesza, L. Motyka, Threshold resummation for squarkantisquark and gluino-pair production at the LHC. Phys. Rev. Lett. 102, 111802 (2009). arXiv:0807.2405 [hep-ph]
46. W. Beenakker et al., Squark and gluino production at hadron colliders. Nucl. Phys. B 492, 51 (1997). arXiv:hep-ph/9610490

47. J. Butterworth et al., PDF4LHC recommendations for LHC Run II. J. Phys. G 43, 023001 (2016). arXiv:1510.03865 [hep-ph]

48. T. Gleisberg, S. Höche, Comix, a new matrix element generator. JHEP 12, 039 (2008). arXiv:0808.3674 [hep-ph]

49. F. Cascioli, P. Maierhöfer, S. Pozzorini, Scattering amplitudes with open loops. Phys. Rev. Lett. 108, 111601 (2012). arXiv:1111.5206 [hep-ph]

50. A. Denner, S. Dittmaier, L. Hofer, Collier: A fortran-based complex one-loop library in extended regularizations. Comput. Phys. Commun. 212, 220 (2017). arXiv: 1604.06792 [hep-ph]

51. S. Schumann, F. Krauss, A parton shower algorithm based on Catani-Seymour dipole factorisation. JHEP 03, 038 (2008). arXiv:0709.1027 [hep-ph]

52. S. Höche, F. Krauss, M. Schönherr, F. Siegert, A critical appraisal of NLO+PS matching methods. JHEP 09, 049 (2012). arXiv: 1111.1220 [hep-ph]

53. S. Höche, F. Krauss, M. Schönherr, F. Siegert, QCD matrix elements + parton showers. The NLO case. JHEP 04, 027 (2013). arXiv: 1207.5030 [hep-ph]

54. S. Catani, F. Krauss, R. Kuhn, B.R. Webber, QCD matrix elements + parton showers. JHEP 11, 063 (2001). arXiv:hep-ph/0109231

55. S. Höche, F. Krauss, S. Schumann, F. Siegert, QCD matrix elements and truncated showers. JHEP 05, 053 (2009). arXiv:0903.1219 [hep-ph]

56. D.J. Lange, The EvtGen particle decay simulation package. Nucl. Instrum. Methods A 462, 152 (2001)

57. L. Lönnblad, S. Prestel, Merging multi-leg NLO matrix elements with parton showers. JHEP 03, 166 (2013). arXiv:1211.7278 [hep$\mathrm{ph}]$

58. S. Alioli, P. Nason, C. Oleari, E. Re, A general framework for implementing NLO calculations in shower Monte Carlo programs: the POWHEG BOX. JHEP 06, 043 (2010). arXiv:1002.2581 [hep$\mathrm{ph}]$

59. S. Frixione, P. Nason, C. Oleari, Matching NLO QCD computations with parton shower simulations: the POWHEG method. JHEP 11, 070 (2007). arXiv:0709.2092 [hep-ph]

60. S. Frixione, P. Nason, G. Ridolfi, A positive-weight next-toleading-order Monte Carlo for heavy flavour hadroproduction. JHEP 09, 126 (2007). arXiv:0707.3088 [hep-ph]

61. P. Nason, A new method for combining NLO QCD with shower Monte Carlo algorithms. JHEP 11, 040 (2004). arXiv:hep-ph/0409146

62. T. Sjöstrand et al., An introduction to PYTHIA 8.2. Comput. Phys. Commun. 191, 159 (2015). http://www.sciencedirect.com/science/ article/pii/S0010465515000442 (issn: 0010-4655)

63. ATLAS Collaboration, ATLAS Pythia 8 tunes to $7 \mathrm{TeV}$ data, ATLPHYS-PUB-2014-021 (2014). https://cds.cern.ch/record/1966419

64. M. Czakon, A. Mitov, Top++: a program for the calculation of the top-pair cross-section at hadron colliders. Comput. Phys. Commun. 185, 2930 (2014). arXiv:1112.5675 [hep-ph]

65. E. Re, Single-top Wt-channel production matched with parton showers using the POWHEG method. Eur. Phys. J. C 71, 1547 (2011). arXiv:1009.2450 [hep-ph]

66. R. Frederix, E. Re, P. Torrielli, Single-top t-channel hadroproduction in the four-flavour scheme with POWHEG and aMC@NLO. JHEP 09, 130 (2012). arXiv:1207.5391 [hep-ph]

67. S. Alioli, P. Nason, C. Oleari, E. Re, NLO single-top production matched with shower in POWHEG:s- and t-channel contributions. JHEP 09, 111 (2009). [Erratum: JHEP 02, 011 (2010)]. arXiv:0907.4076 [hep-ph]

68. M. Aliev et al., HATHOR-HAdronic Top and Heavy quarks crOss section calculatoR. Comput. Phys. Commun. 182, 1034 (2011). arXiv:1007.1327 [hep-ph] 
69. E. Bothmann et al., Event generation with Sherpa 2.2. SciPost Phys. 7, 034 (2019). arXiv:1905.09127 [hep-ph]

70. C. Anastasiou, L.J. Dixon, K. Melnikov, F. Petriello, High precision QCD at hadron colliders: electroweak gauge boson rapidity distributions at NNLO. Phys. Rev. D 69, 094008 (2004). arXiv:hep-ph/0312266

71. P. Bärnreuther, M. Czakon, A. Mitov, Percent level precision physics at the tevatron: first genuine NNLO QCD corrections to $\mathrm{q} \overline{\mathrm{q}} \rightarrow \overline{\mathrm{t}}+$ X. Phys. Rev. Lett. 109, 132001 (2012). arXiv: 1204.5201 [hep-ph]

72. ATLAS Collaboration, Vertex Reconstruction Performance of the ATLAS Detector at v s $=13 \mathrm{TeV}$, ATL-PHYS-PUB-2015-026 (2015). https://cds.cern.ch/record/2037717

73. ATLAS Collaboration, Selection of jets produced in $13 \mathrm{TeV}$ protonproton collisions with the ATLAS detector, ATLAS-CONF-2015029 (2015). https://cds.cern.ch/record/2037702

74. ATLAS Collaboration, Electron and photon performance measurements with the ATLAS detector using the 2015-2017 LHC protonproton collision data. JINST 14, P12006 (2019). arXiv: 1908.00005 [hep-ex]

75. ATLAS Collaboration, Muon reconstruction performance of the ATLAS detector in proton-proton collision data at $\mathrm{v} \mathrm{s}=13 \mathrm{TeV}$. Eur. Phys. J. C 76, 292 (2016). arXiv:1603.05598 [hep-ex]

76. ATLAS Collaboration, Topological cell clustering in the ATLAS calorimeters and its performance in LHC Run 1. Eur. Phys. J. C 77, 490 (2017). arXiv:1603.02934 [hep-ex]

77. M. Cacciari, G.P. Salam, G. Soyez, The anti-kt jet clustering algorithm. JHEP 04, 063 (2008). arXiv:0802.1189 [hep-ph]

78. ATLAS Collaboration, Performance of pile-up mitigation techniques for jets in pp collisions at $\mathrm{vs}=8 \mathrm{TeV}$ using the ATLAS detector. Eur. Phys. J. C 76, 581 (2016). arXiv:1510.03823 [hepex]

79. M. Cacciari, G.P. Salam, G. Soyez, FastJet user manual. Eur. Phys. J. C 72, 1896 (2012). arXiv:1111.6097 [hep-ph]

80. ATLAS Collaboration, ATLAS b-jet identification performance and efficiency measurement with $\mathrm{tt}$ events in pp collisions at vs = 13 TeV. Eur. Phys. J. C 79, 970 (2019). arXiv:1907.05120 [hepex]

81. ATLAS Collaboration, Optimisation and performance studies of the ATLAS b-tagging algorithms for the 2017-18 LHC run, ATLPHYS-PUB-2017-013 (2017). https://cds.cern.ch/record/2273281

82. M. Cacciari, G.P. Salam, G. Soyez, The catchment area of jets. JHEP 04, 005 (2008). arXiv:0802.1188 [hep-ph]

83. ATLAS Collaboration, Measurement of the photon identification efficiencies with the ATLAS detector using LHC Run 2 data collected in 2015 and 2016. Eur. Phys. J. C 79, 205 (2019). arXiv: 1810.05087 [hep-ex]
84. ATLAS Collaboration, Performance of missing transverse momentum reconstruction with the ATLAS detector using proton-proton collisions at v s = 13 TeV. Eur. Phys. J. C 78, 903 (2018). arXiv: 1802.08168 [hep-ex]

85. ATLAS Collaboration, E miss T performance in the ATLAS detector using 2015-2016 LHC pp collisions, ATLAS-CONF-2018-023 (2018). https://cds.cern.ch/record/2625233

86. ATLAS Collaboration, Performance of the missing transverse momentum triggers for the ATLAS detector during Run-2 data taking. JHEP 08, 080 (2020). arXiv:2005.09554 [hep-ex]

87. C. Chen, New approach to identifying boosted hadronically decaying particles using jet substructure in its center-of-mass frame. Phys. Rev. D 85, 034007 (2012). arXiv:1112.2567 [hep-ph]

88. J. Bellm et al., Herwig 7.0/Herwig++ 3.0 release note. Eur. Phys. J. C 76, 196 (2016). arXiv:1512.01178 [hep-ph]

89. ATLAS Collaboration, Simulation of top-quark production for the ATLAS experiment at $\mathrm{v} \mathrm{s}=13 \mathrm{TeV}$, ATL-PHYS-PUB-2016-004 (2016). https://cds.cern.ch/record/2120417

90. ATLAS Collaboration, Multi-boson simulation for $13 \mathrm{TeV}$ ATLAS analyses, ATL-PHYS-PUB-2016-002 (2016). https://cds.cern.ch/ record $/ 2119986$

91. ATLAS Collaboration, Jet energy scale measurements and their systematic uncertainties in proton-proton collisions at $\mathrm{v} \mathrm{s}=13$ TeV with the ATLAS detector. Phys. Rev. D 96, 072002 (2017). arXiv:1703.09665 [hep-ex]

92. ATLAS Collaboration, Calibration of the b-tagging efficiency on charm jets using a sample of $\mathrm{W}+\mathrm{c}$ events with $\mathrm{v} \mathrm{s}=13 \mathrm{TeV}$ ATLAS data, ATLAS-CONF-2018-055 (2018). https://cds.cern.ch/record/ 2652195

93. ATLAS Collaboration, Calibration of light-flavour b-jet mistagging rates using ATLAS proton-proton collision data at $\mathrm{v} \mathrm{s}=13$ TeV, ATLAS-CONF-2018-006 (2018). https://cds.cern.ch/record/ 2314418

94. M. Baak et al., HistFitter software framework for statistical data analysis. Eur. Phys. J. C 75, 153 (2015). arXiv:1410.1280 [hep-ex]

95. G. Cowan, K. Cranmer, E. Gross, O. Vitells, Asymptotic formulae for likelihood-based tests of new physics. Eur. Phys. J. C 71, 1554 (2011). arXiv:1007.1727 [physics.data-an]. [Erratum: Eur. Phys. J. C 73, 2501 (2013)]

96. A.L. Read, Presentation of search results: the CLS technique. J. Phys. G 28, 2693 (2002)

97. ATLAS Collaboration, ATLAS Computing Acknowledgements, ATL-SOFT-PUB-2020-001. https://cds.cern.ch/record/2717821 


\section{ATLAS Collaboration}

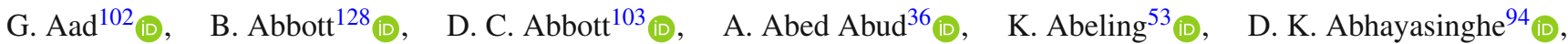

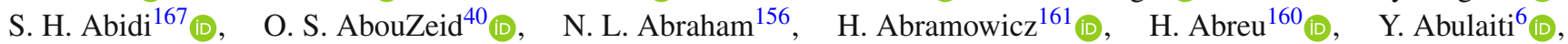

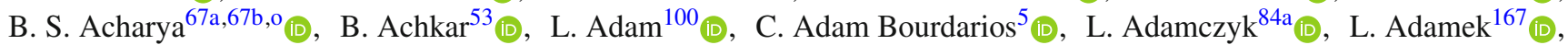

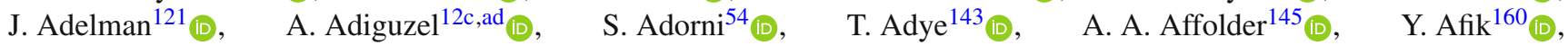
C. Agapopoulou ${ }^{65}$ (D) M. N. Agaras $^{38}$ (D), A. Aggarwal ${ }^{119}$ (D), C. Agheorghiesei ${ }^{27 c}$ (D), J. A. Aguilar-Saavedra ${ }^{139 a, 139 f, a c}$ (D),

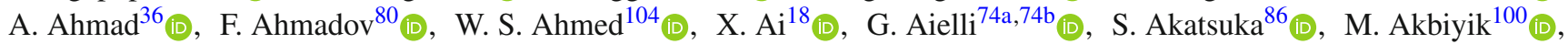

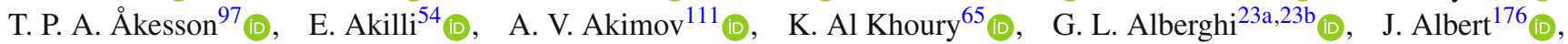

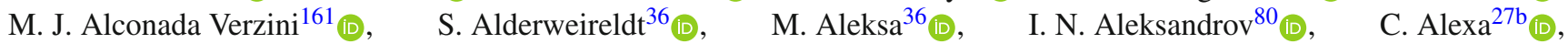

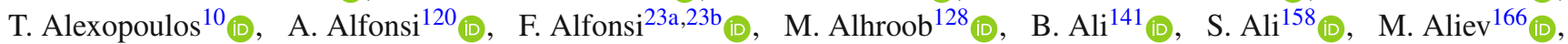

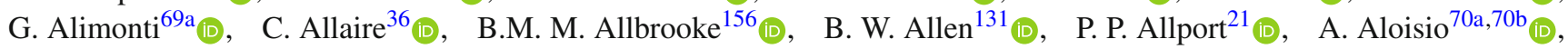

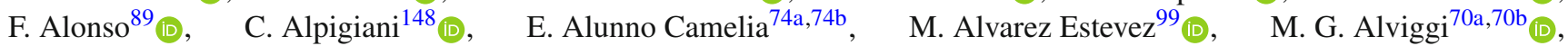
Y. Amaral Coutinho ${ }^{81 b}$ (D) A. Ambler ${ }^{104}$ (D) , L. Ambroz ${ }^{134}$ (D) , C. Amelung ${ }^{36}$, D. Amidei ${ }^{106}$ (D) , S. P. Amor Dos Santos ${ }^{139 a}$ (D),

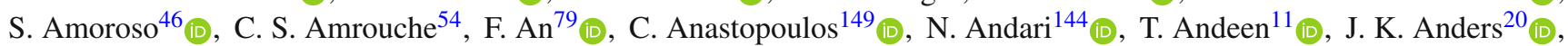

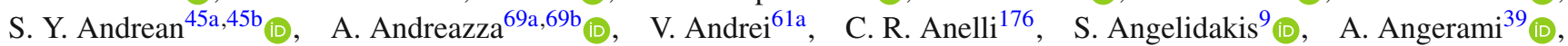

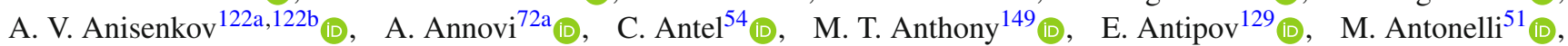

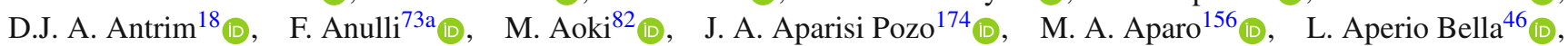

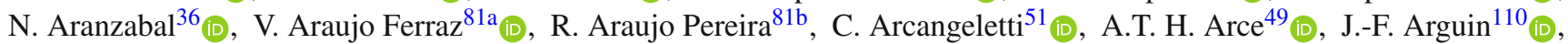

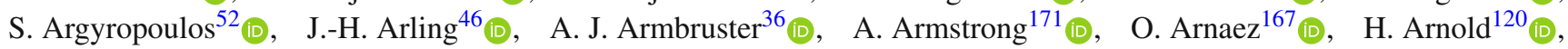

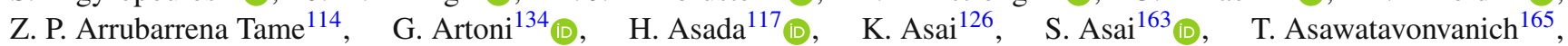

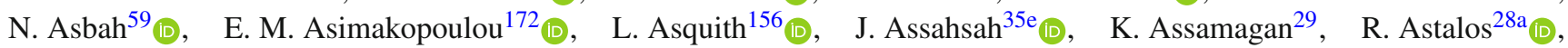
R. J. Atkin ${ }^{33 a}$ (D) , M. Atkinson ${ }^{173}$, N. B. Atlay ${ }^{19}$ (D) H. Atmani ${ }^{65}$, P. A. Atmasiddha ${ }^{106}$, K. Augsten ${ }^{141}$ (I) , V. A. Austrup ${ }^{182}$ (D),

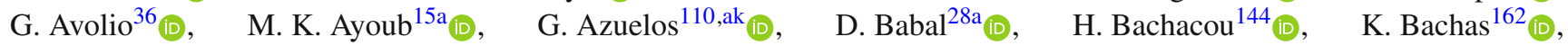

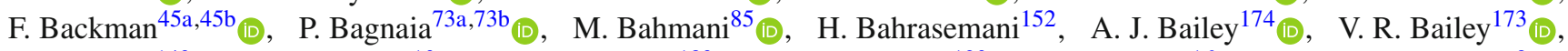

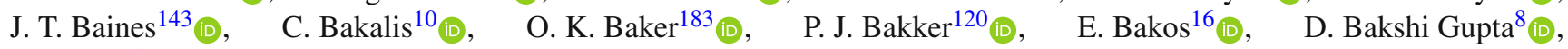
S. Balaji ${ }^{157}$ (D), R. Balasubramanian ${ }^{120}$ (D), E. M. Baldin ${ }^{122 a, 122 b}$ (D) $^{2}$ P. Balek ${ }^{180}$ (D), F. Balli ${ }^{144}$ (D), W. K. Balunas ${ }^{134}$ (D), J. Balz ${ }^{100}$ (D), E. Banas ${ }^{85}$ (D), M. Bandieramonte ${ }^{138}$ (D), A. Bandyopadhyay ${ }^{19}$ (D), Sw. Banerjee ${ }^{181, j}$ (D), L. Barak ${ }^{161}$ (D),

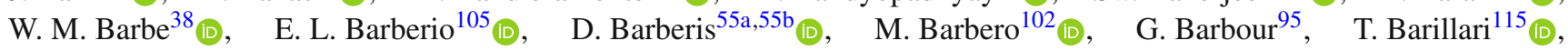

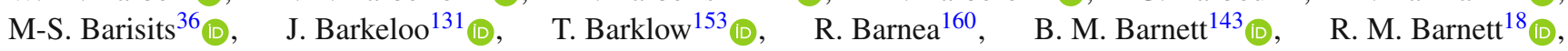

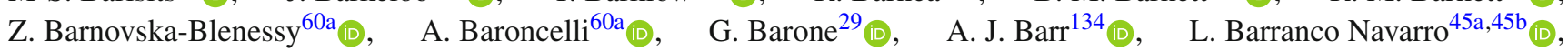

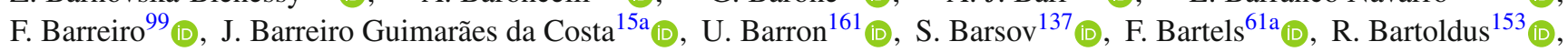

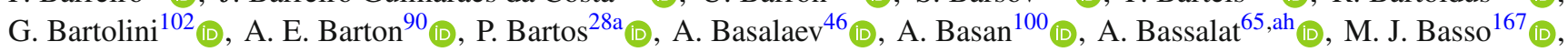

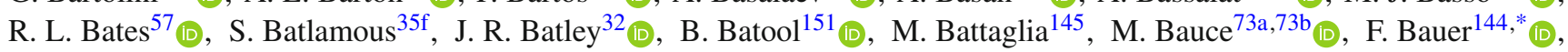

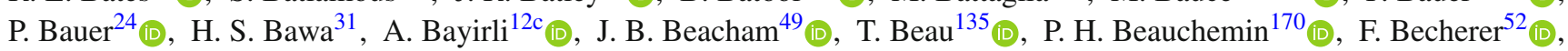

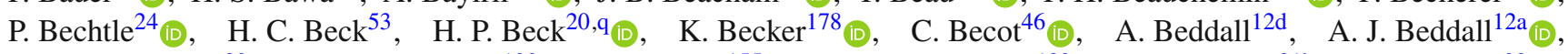

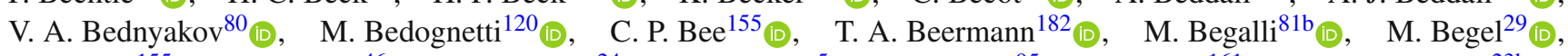

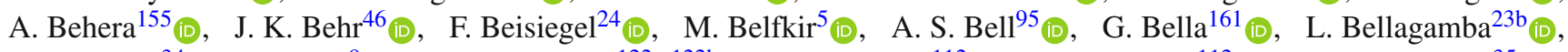

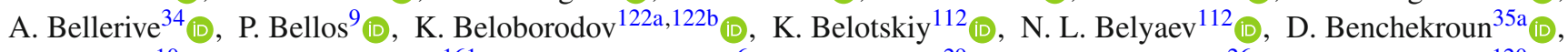

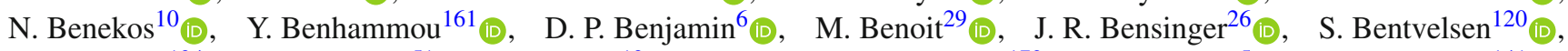
L. Beresford ${ }^{134}$ (I), M. Beretta ${ }^{51}$ (D), D. Berge ${ }^{19}$ (D), E. Bergeaas Kuutmann ${ }^{172}$ (I), N. Berger ${ }^{5}$ (D), B. Bergmann ${ }^{141}$ (D),

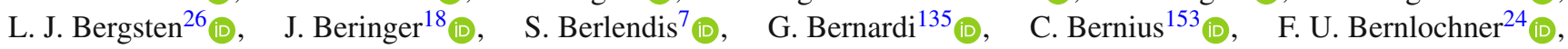
T. Berry ${ }^{94}$ (D), P. Berta ${ }^{100}{ }^{(D)}, \quad$ A. Berthold ${ }^{48}$ (D), I. A. Bertram ${ }^{90}$ (D), O. Bessidskaia Bylund ${ }^{182}$ (D), N. Besson ${ }^{144}$ (D), S. Bethke ${ }^{115}$ (D), A. Betti ${ }^{42}$ (I), A. J. Bevan ${ }^{93}$ (D), J. Beyer ${ }^{115}$ (D), S. Bhatta ${ }^{155}$ (D), D. S. Bhattacharya ${ }^{177}$ (I), P. Bhattarai ${ }^{26}$,

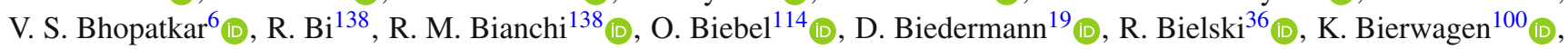

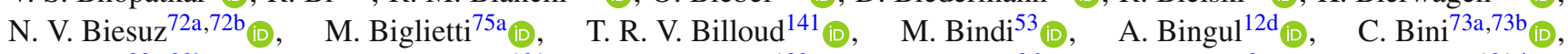

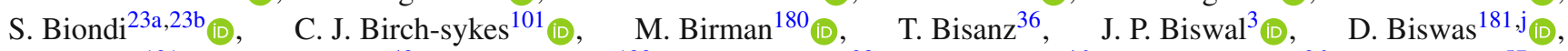

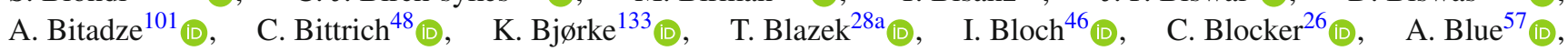

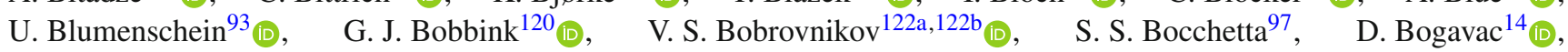

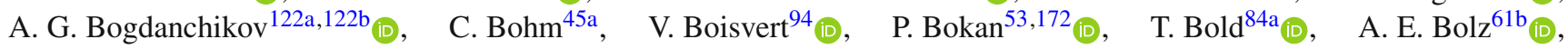
M. Bomben ${ }^{135}$ (D), M. Bona ${ }^{93}$ (D), J. S. Bonilla ${ }^{131}$ (D), M. Boonekamp ${ }^{144}$ (D), C. D. Booth ${ }^{94}$ (D), A. G. Borbély ${ }^{57}$ (D),

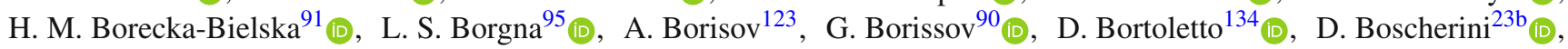
M. Bosman ${ }^{14}$ (i), J. D. Bossio Sola ${ }^{104}$ (i), K. Bouaouda ${ }^{\left.35 a_{(}\right)} \quad$ J. Boudreau $^{138}$ (i), E. V. Bouhova-Thacker ${ }^{90}$ (D), 


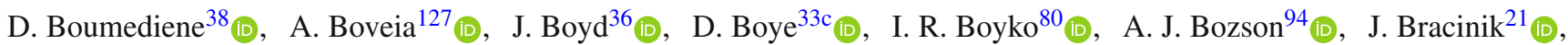

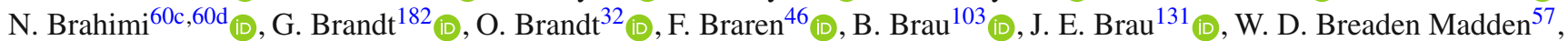

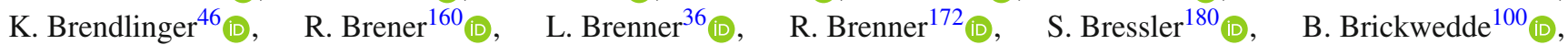

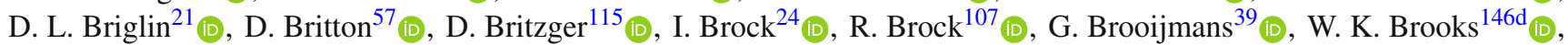

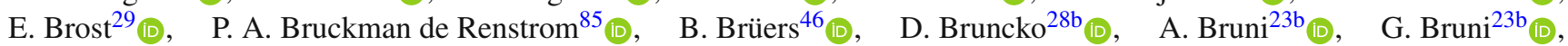

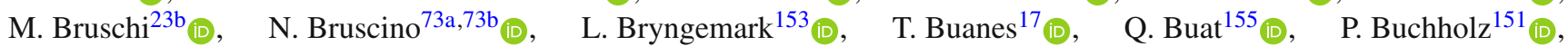
A. G. Buckley ${ }^{57}$ (D), I. A. Budagov ${ }^{80}$ (D), M. K. Bugge ${ }^{133}$ (D), O. Bulekov ${ }^{112}$ (D), B. A. Bullard ${ }^{59}$ (D), T. J. Burch ${ }^{121}$ (D),

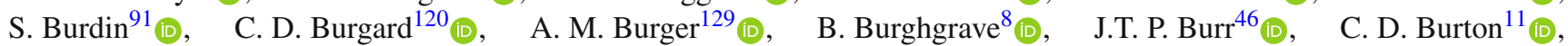

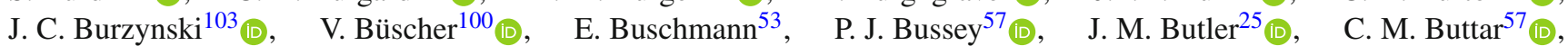
J. M. Butterworth ${ }^{95}$ (D), P. Butti ${ }^{36}$, W. Buttinger ${ }^{143}$ (D), C. J. Buxo Vazquez ${ }^{107}$, A. Buzatu ${ }^{158}$ (D), A. R. Buzykaev ${ }^{122 a, 122 b}$ (D), $^{\text {(D) }}$ G. Cabras ${ }^{23 a}, 23 b$ (D), S. Cabrera Urbán ${ }^{174}$ (D), D. Caforio ${ }^{56}$ (D) H. Cai ${ }^{138}$ (D), V. M. M. Cairo ${ }^{153}$ (D), O. Cakir ${ }^{4 a}$ (D), N. Calace ${ }^{36}$ (D) P. Calafiura ${ }^{18}$ (D) G. Calderini ${ }^{135}$ (D) P. Calfayan ${ }^{66}$ (D) , G. Callea ${ }^{57}$ (D) L. P. Caloba ${ }^{81 b}$, A. Caltabiano ${ }^{74 a, 74 b}$, S. Calvente Lopez ${ }^{99}$ (D), D. Calvet ${ }^{38}$ (D), S. Calvet ${ }^{38}$ (D), T. P. Calvet ${ }^{102}{ }_{(\mathbb{D})}$, M. Calvetti ${ }^{72 a, 72 b}$ (D), R. Camacho Toro ${ }^{135}{ }_{(\mathbb{D})}$,

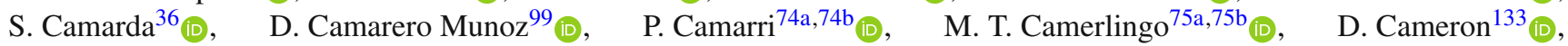

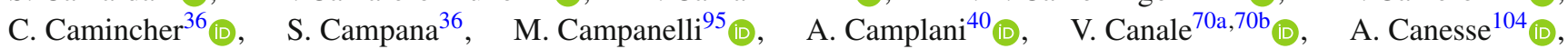

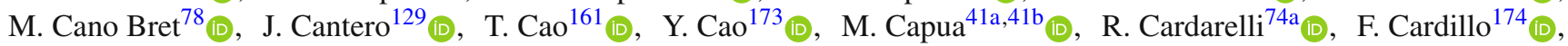

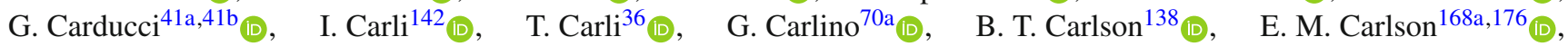

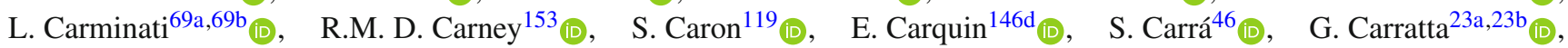

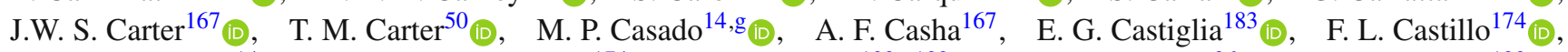
L. Castillo Garcia ${ }^{14}$ (D), V. Castillo Gimenez ${ }^{174}$ (D), N. F. Castro ${ }^{139 a, 139 e} e_{\mathbb{C}}, \quad$ A. Catinaccio $^{36}$ (D), J. R. Catmore ${ }^{133}$ (D),

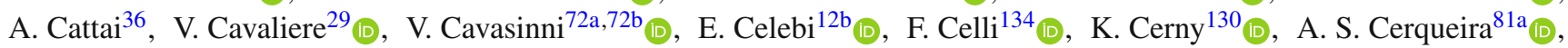
A. Cerri ${ }^{156}$ (D) L. Cerrito ${ }^{74 a, 74 b}$ (D) F. Cerutti ${ }^{18}$ (D) A. Cervelli ${ }^{23 a, 23 b}{ }_{(D)}$, S. A. Cetin ${ }^{12 b}$ (D) Z. Chadi ${ }^{35 a}$, D. Chakraborty ${ }^{121}$ (D), J. Chan ${ }^{181}$ (D), W. S. Chan ${ }^{120}$ (D), W. Y. Chan ${ }^{91}$ (D), J. D. Chapman ${ }^{32}$ (D) $\quad$ B. Chargeishvili ${ }^{159 b}$ (D), D. G. Charlton ${ }^{21}$ (D),

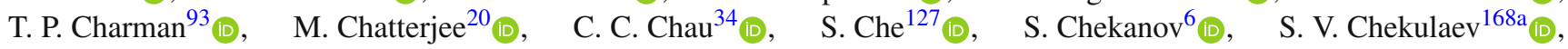

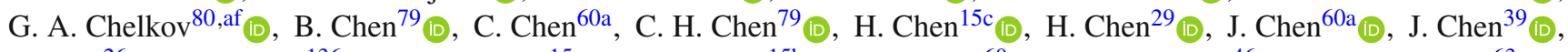

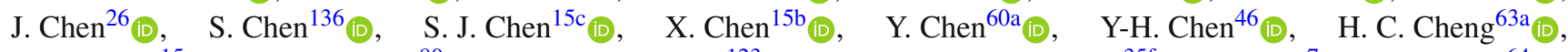
H. J. Cheng ${ }^{15 a}$ (D) A. Cheplakov ${ }^{80}$ (D) E. Cheremushkina ${ }^{123}$ (D) R. Cherkaoui El Moursli ${ }^{35 f}$ (ID, E. Cheu ${ }^{7}$, K. K. Cheung ${ }^{64}$ (D),

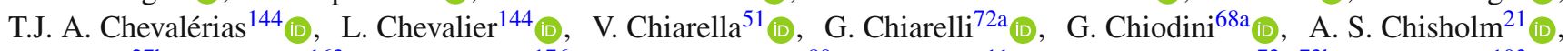

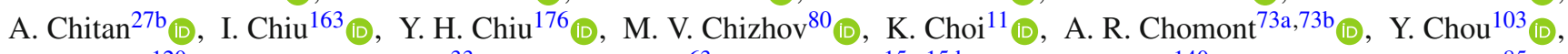

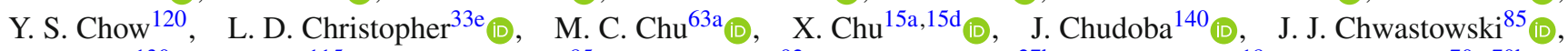

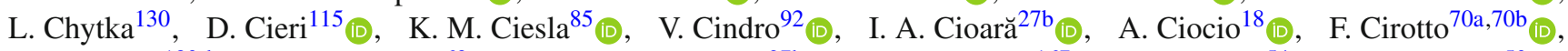

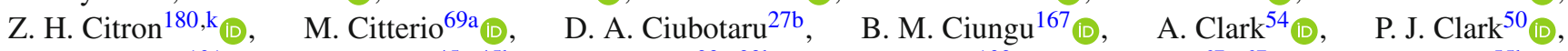

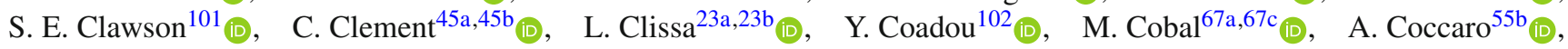
J. Cochran ${ }^{79}$, R. Coelho Lopes De $\mathrm{Sa}^{103}$ (I),$\quad$ H. Cohen ${ }^{161}, \quad$ A. E. C. Coimbra ${ }^{36}$ (I), B. Cole ${ }^{39}$ (D), A. P. Colijn ${ }^{120}$,

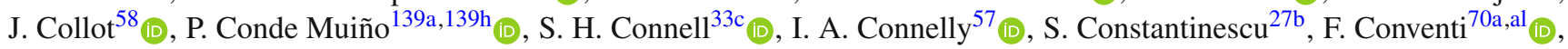

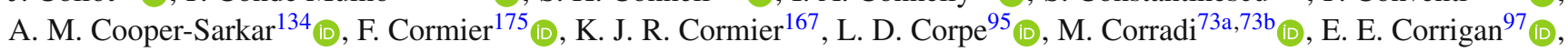

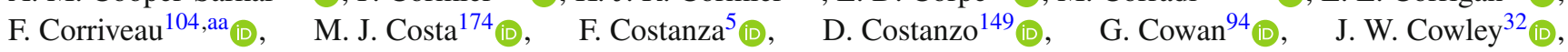
J. Crane ${ }^{101}$ (D) K. Kranmer ${ }^{125}$ (D), R. A. Creager ${ }^{136}$ (D), S. Crépé-Renaudin ${ }^{58}$ (D) F. Crescioli ${ }^{135}$ (D), M. Cristinziani ${ }^{24}$ (D),

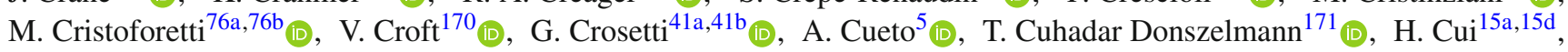

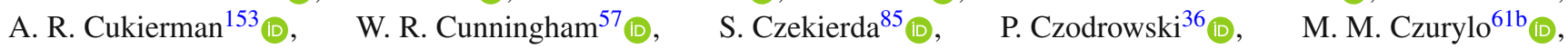

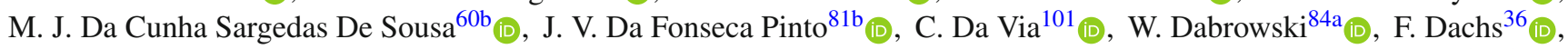

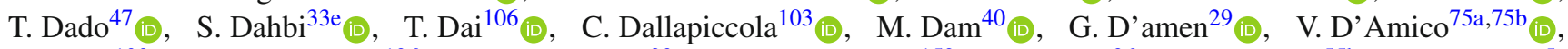

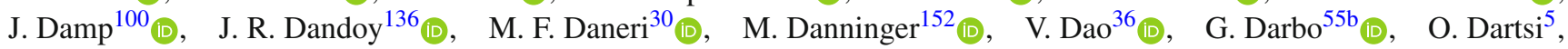

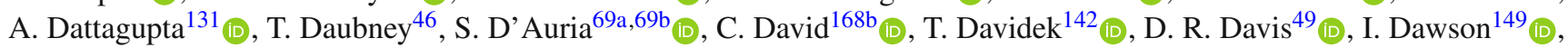

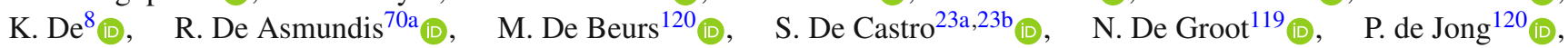

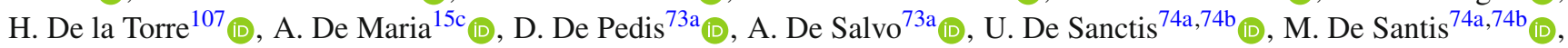

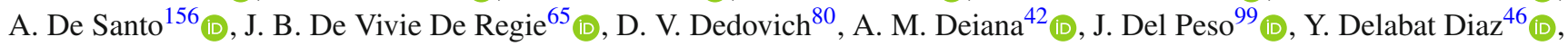

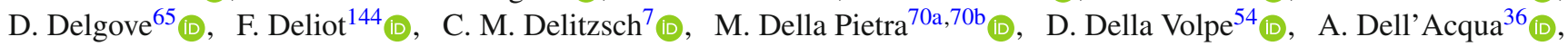

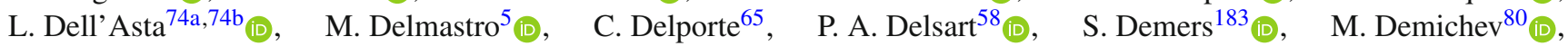

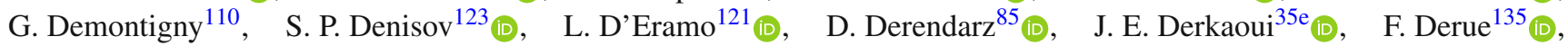

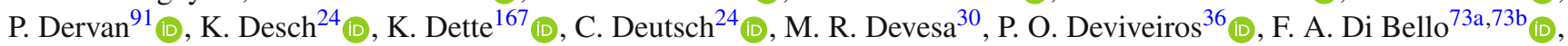

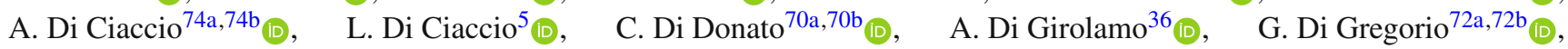

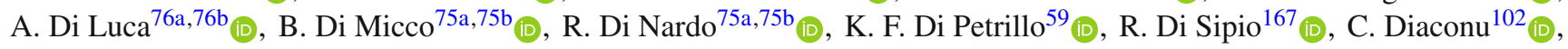




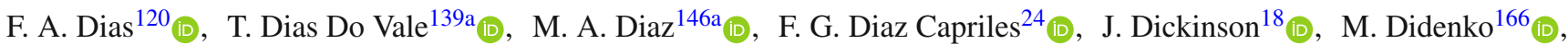

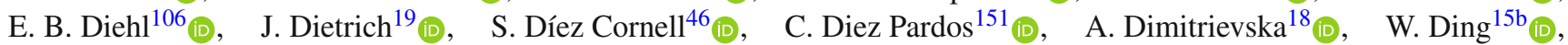

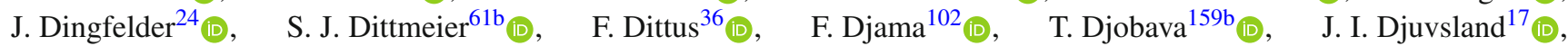
M.A. B. Do Vale ${ }^{147}$ (D), M. Dobre ${ }^{27 b}$ (D),$\quad$ D. Dodsworth ${ }^{26}$ (D) $\quad$ C. Doglioni ${ }^{97}$ (D), J. Dolejsi ${ }^{142}$ (D), Z. Dolezal ${ }^{142}$ (D),

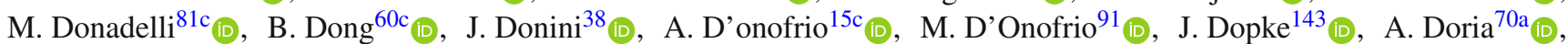

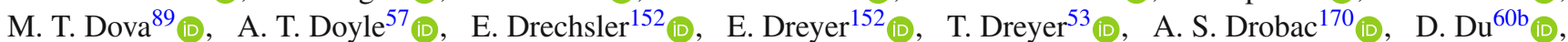

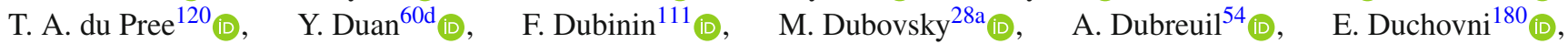

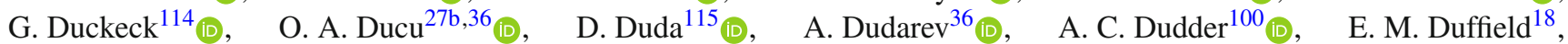

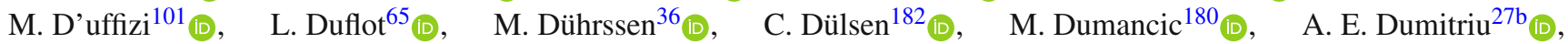

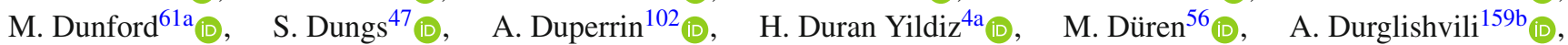

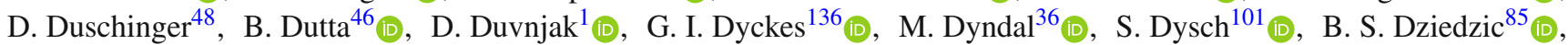
M. G. Eggleston ${ }^{49}$, T. Eifert $^{8}$ (D), G. Eigen $^{17}$ (D), K. Einsweiler ${ }^{18}$ (D), T. Ekelof ${ }^{172}$ (D) H. El Jarrari ${ }^{35 f}$ (D), V. Ellajosyula ${ }^{172}$ (D), M. Ellert ${ }^{172}$ (D) , F. Ellinghaus ${ }^{182}$ (D), A. A. Elliot $^{93}$ (D), N. Ellis $^{36}$ (D) J. Elmsheuser ${ }^{29}$ (D) M. Elsing ${ }^{36}$ (D), D. Emeliyanov ${ }^{143}$ (D),

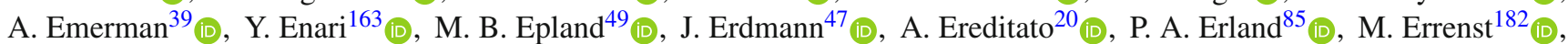

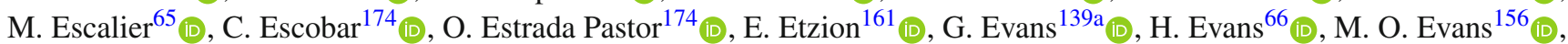

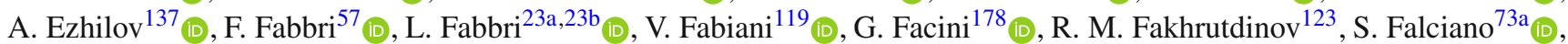

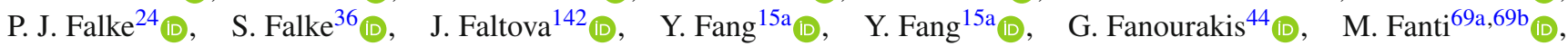

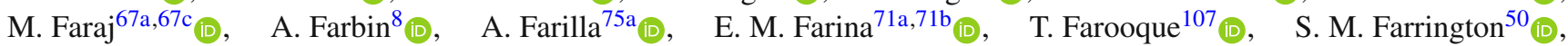
P. Farthouat ${ }^{36}$ (D), F. Fassi ${ }^{35 f}$ (I), P. Fassnacht ${ }^{36}$ (D), D. Fassouliotis ${ }^{9}$ (D), M. Faucci Giannelli ${ }^{50}$ (D), W. J. Fawcett ${ }^{32}$ (D),

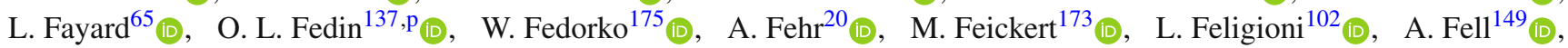
C. Feng ${ }^{60 b}{ }_{(\mathbb{D})}$, M. Feng $^{49}$ (D), M. J. Fenton ${ }^{171}$ (D), A. B. Fenyuk ${ }^{123}$, S. W. Ferguson ${ }^{43}$ (D), J. Ferrando ${ }^{46}$ (D), A. Ferrari ${ }^{172}$ (D),

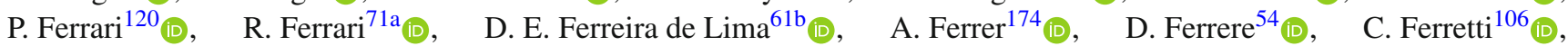

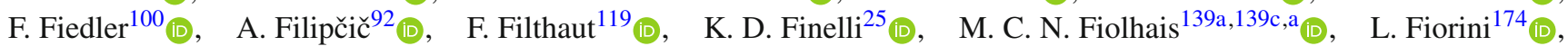

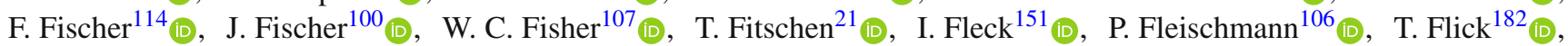

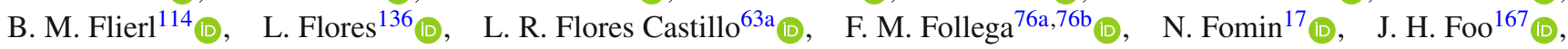

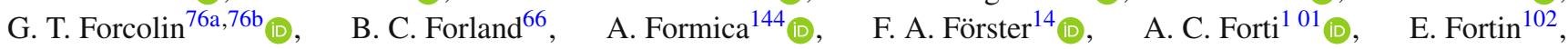

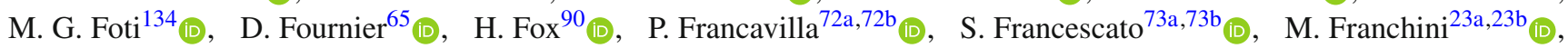

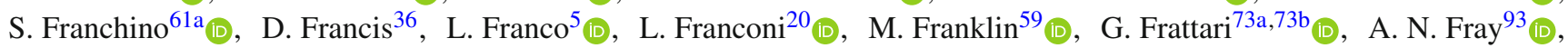

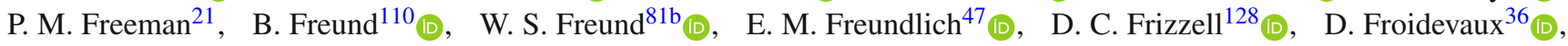
J. A. Frost ${ }^{134}\left(\mathbb{D}, \quad\right.$ M. Fujimoto ${ }^{126}$ (D), C. Fukunaga ${ }^{164}$ (D), E. Fullana Torregrosa ${ }^{174}$ (D), T. Fusayasu ${ }^{116}, \quad$ J. Fuster ${ }^{174}$ (D), A. Gabrielli ${ }^{23 a, 23 b}{ }_{(\mathbb{D}}, \quad$ A. Gabrielli ${ }^{36}$ (D), S. Gadatsch ${ }^{54}$ (D), P. Gadow ${ }^{115}$ (D), G. Gagliardi ${ }^{55 a, 55 b}$ (D), L. G. Gagnon ${ }^{110}{ }_{(\mathbb{D})}$, G. E. Gallardo ${ }^{134}$ (D), E. J. Gallas ${ }^{134}$ (I),$\quad$ B. J. Gallop ${ }^{143}$ (D), R. Gamboa Goni ${ }^{93}$ (I), K. K. Gan ${ }^{127}$ (D), S. Ganguly ${ }^{180}$ (D),

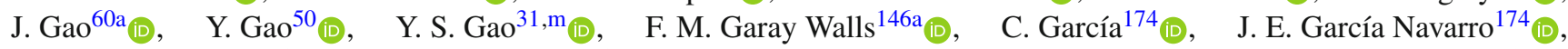

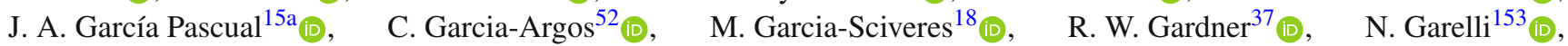

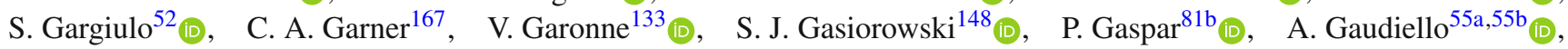

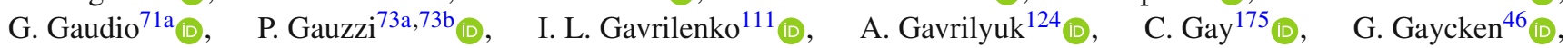

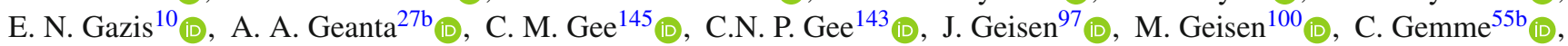

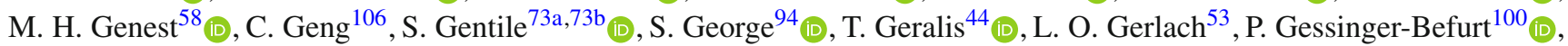

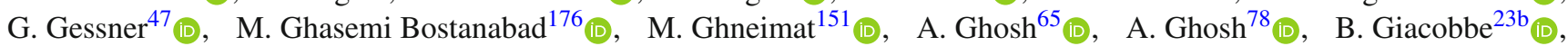

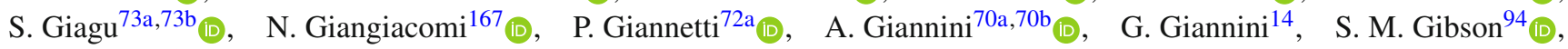

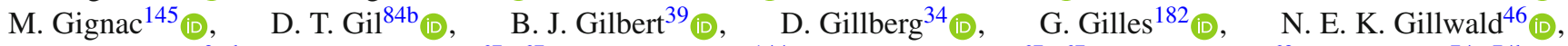

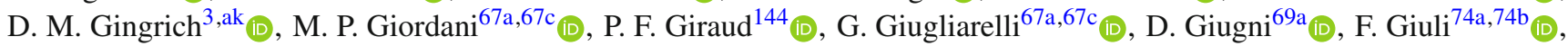

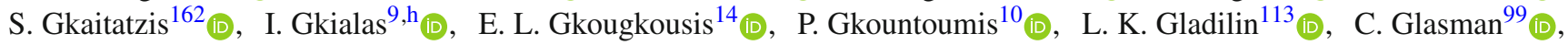

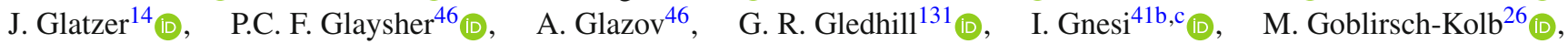
D. Godin ${ }^{110}, \quad$ S. Goldfarb ${ }^{105}$ (D),$\quad$ T. Golling ${ }^{54}$ (D) , D. Golubkov ${ }^{123}$ (D), A. Gomes ${ }^{139 a}, 139 b$ (D), R. Goncalves Gama ${ }^{53}$ (D),

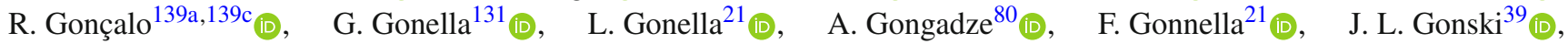
S. González de la Hoz ${ }^{174}$ (D), S. Gonzalez Fernandez ${ }^{14}$ (D), R. Gonzalez Lopez ${ }^{91}$ (D), $\quad$ C. Gonzalez Renteria ${ }^{18}$ (D), R. Gonzalez Suarez ${ }^{172}$ (I) S. Sonzalez-Sevilla ${ }^{54}$ (D), G. R. Gonzalvo Rodriguez ${ }^{174}$ (D), L. Goossens ${ }^{36}$ (D), N. A. Gorasia ${ }^{21}$ (D), P. A. Gorbounov ${ }^{124}$ (D) $\quad$ H. A. Gordon ${ }^{29}$ (D),$\quad$ B. Gorini ${ }^{36}$ (D) $\quad$ E. Gorini ${ }^{68 a}, 68 b$ (D), A. Gorišek ${ }^{92}$ (D),$\quad$ A. T. Goshaw ${ }^{49}$ (D),

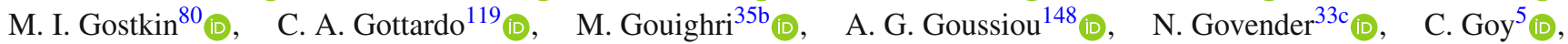

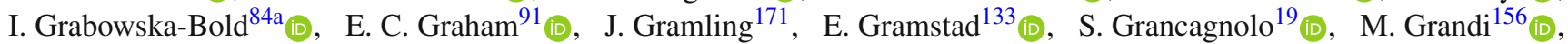

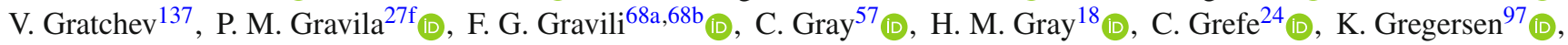

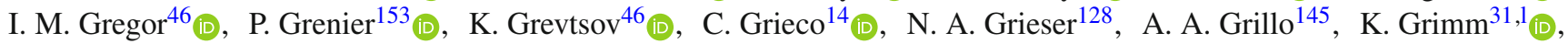




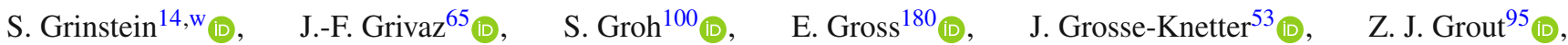
C. Grud ${ }^{106}$, A. Grummer ${ }^{118}$ (D), J. C. Grundy ${ }^{134}$ (D), L. Guan ${ }^{106}$ (D), W. Guan ${ }^{181}$ (D), C. Gubbels ${ }^{175}$ (D), J. Guenther ${ }^{77}$ (D), A. Guerguichon ${ }^{65}$ (D), J.G. R. Guerrero Rojas ${ }^{174}$ (D), F. Guescini ${ }^{115}$ (D), D. Guest ${ }^{77}$ (D), R. Gugel ${ }^{100}$ (D), A. Guida $^{46}$ (D),

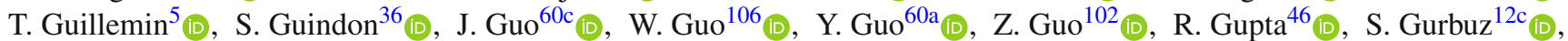

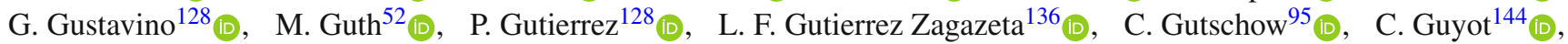

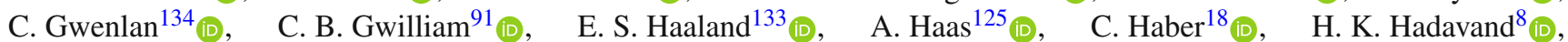

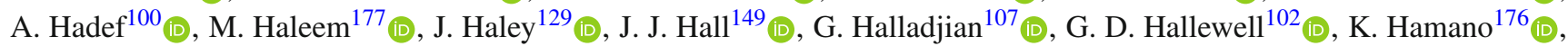

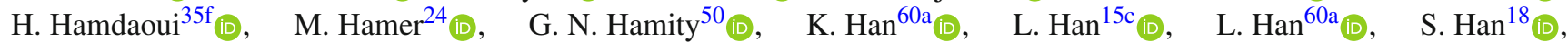

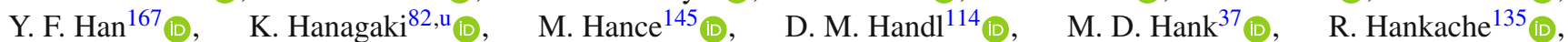
E. Hansen ${ }^{97}$ (D), J. B. Hansen ${ }^{40}$ (D), J. D. Hansen ${ }^{40}$ (D) $\quad$ M. C. Hansen ${ }^{24}$ (D), P. H. Hansen ${ }^{40}$ (D), $\quad$ E. C. Hanson ${ }^{101}{ }_{(D)}$, K. $\operatorname{Hara}^{169}$ (D), T. Harenberg ${ }^{182}$ (D), S. Harkusha ${ }^{108}$ (D), P. F. Harrison ${ }^{178}$ ， N. M. $\operatorname{Hartman}^{153}$ (D), N. M. $\operatorname{Hartmann}^{114}$ (D),

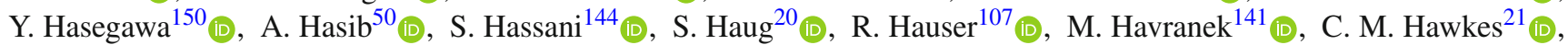

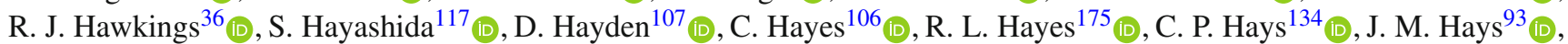

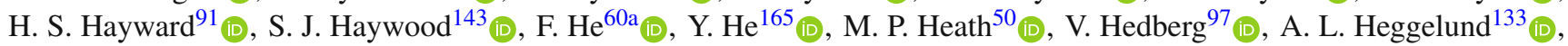

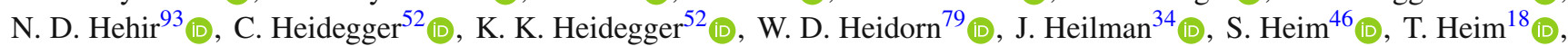

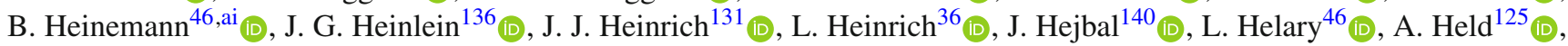
S. Hellesund ${ }^{133}$ (D), C. M. Helling ${ }^{145}$ (D), S. Hellman ${ }^{45 a, 45 b}$ (D), C. Helsens ${ }^{36}$ (D), R.C. W. Henderson ${ }^{90}$, L. Henkelmann ${ }^{32}$ (D),

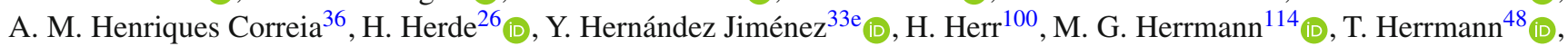

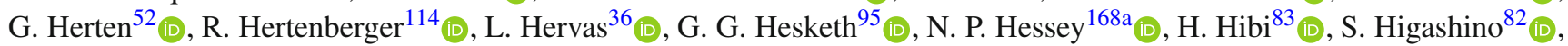

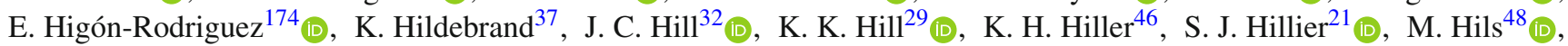
I. Hinchliffe ${ }^{18}$ (D), F. Hinterkeuse ${ }^{24}$ (D), M. Hirose ${ }^{132}$ (D), S. Hirose ${ }^{169}$ (D), D. Hirschbuehl ${ }^{182}$ (D), B. Hiti ${ }^{92}$ (D), O. Hladik ${ }^{140}$,

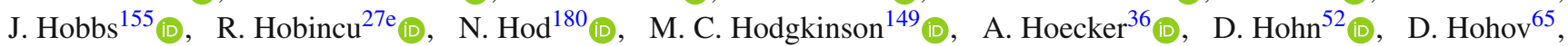
T. Holm ${ }^{24}$ (D), T. R. Holmes ${ }^{37}$ (D), M. Holzbock ${ }^{115}$ (D), L.B.A. H. Hommels ${ }^{32}$ (D), T. M. Hong ${ }^{138}$ (D), J. C. Honig ${ }^{52}$ (D), A. Hönle ${ }^{115}$ (D), B. H. Hooberman ${ }^{173}$ (D), W. H. Hopkins ${ }^{6}$ (D) Y. Horii ${ }^{117}$ (D), P. Horn ${ }^{48}$ (D) L. A. Horyn ${ }^{37}$ (D), S. Hou ${ }^{158}$ (D), A. Hoummada ${ }^{35 a}$, J. Howarth ${ }^{57}$ (D) J. Hoya ${ }^{89}$ (D), M. Hrabovsky ${ }^{130}$ (D), J. Hrivnac ${ }^{65}$, A. Hrynevich ${ }^{109}$ (D), T. Hryn'ova ${ }^{5}$ (D),

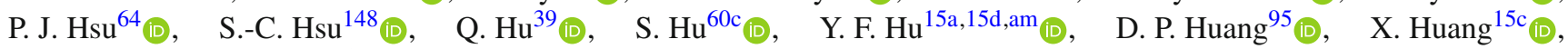

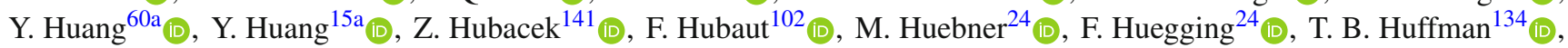

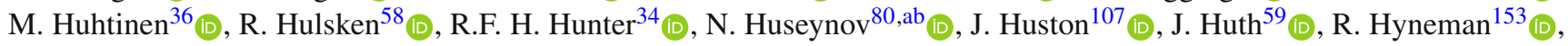

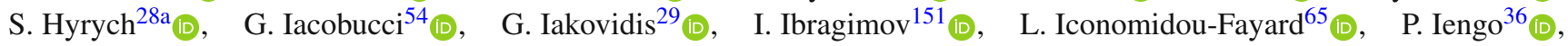
R. Ignazzi ${ }^{40}$, R. Iguchi ${ }^{163}$ (D), T. Iizawa ${ }^{54}$ (D), Y. Ikegami ${ }^{82}$ (D), M. Ikeno ${ }^{82}$ (D) N. Ilic ${ }^{119,167, \text { aa }, ~ F . ~ I l t z s c h e ~}{ }^{48}$, H. Imam $^{35 a}$ (D),

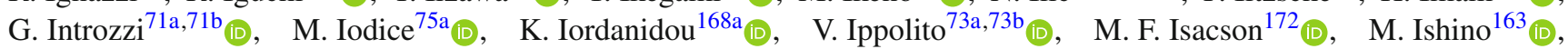

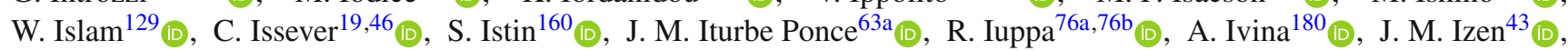

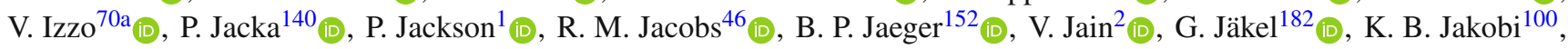

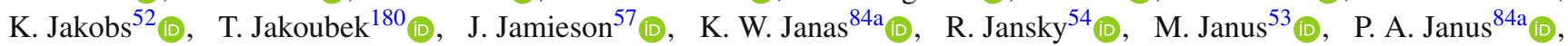
G. Jarlskog ${ }^{97}$ (D), A. E. Jaspan ${ }^{91}$ (i), N. Javadov ${ }^{80, a b}$, T. Javưrek ${ }^{36}$ (D) M. Javurkova ${ }^{103}$ (D), F. Jeanneau ${ }^{144}$ (i), L. Jeanty ${ }^{131}$ (i),

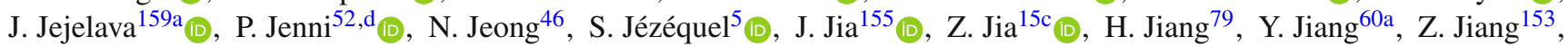

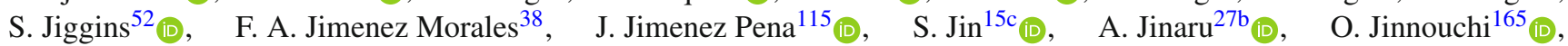

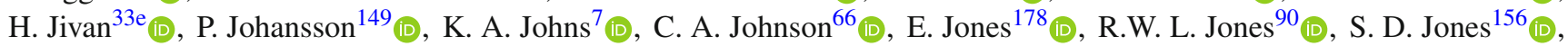

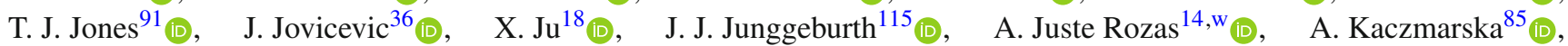
M. Kado ${ }^{73 a}, 73 b$, H. Kagan ${ }^{127}$ (D), M. Kagan ${ }^{153}$ (D), A. Kahn ${ }^{39}$, C. Kahra ${ }^{100}$ (D), T. Kaji ${ }^{179}$ (D), E. Kajomovitz ${ }^{160}$ (D), C. W. Kalderon ${ }^{29}$ (D), A. Kaluza ${ }^{100}, \quad$ A. Kamenshchikov ${ }^{123}$ (D), M. Kaneda ${ }^{163}$ (D), N. J. Kang ${ }^{145}$ (D), S. Kang ${ }^{79}$ (D),

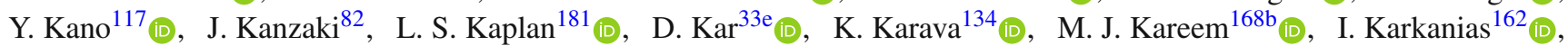

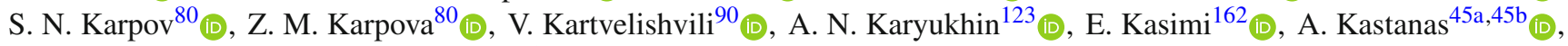

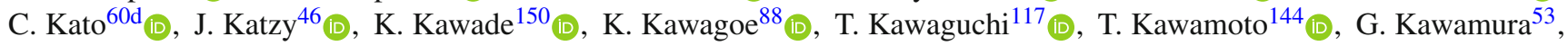

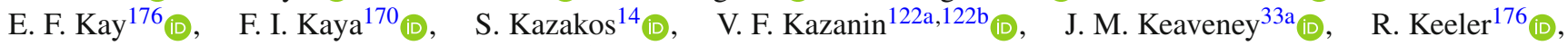

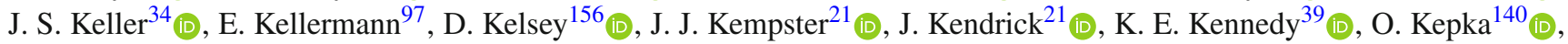
S. Kersten ${ }^{182}$ (D), B. P. Kerševan ${ }^{92}$ (D), S. Ketabchi Haghighat ${ }^{167}$ (D), F. Khalil-Zada ${ }^{13}$, M. Khandoga ${ }^{144}$ (D), A. Khanov ${ }^{129}$ (D),

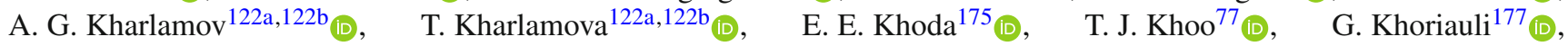

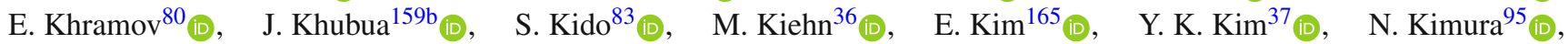
A. Kirchhoff ${ }^{53}$ (D) D. $\operatorname{Kirchmeier}^{48}$ (D) J. Kirk $^{143}$ (D) A. E. Kiryunin ${ }^{115}$ (D), T. Kishimoto ${ }^{163}$ (D), D. P. Kisliuk ${ }^{167}$, V. Kitali ${ }^{46}$ (D), C. Kitsaki ${ }^{10}$ (D), O. Kivernyk ${ }^{24}$ (D), T. Klapdor-Kleingrothaus ${ }^{52}$ (D), M. Klassen ${ }^{61 \mathrm{a}}$ (D)， C. Klein ${ }^{34}$ (D), M. H. Klein ${ }^{106}$ (D),

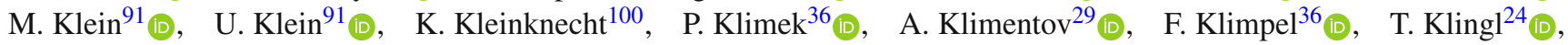

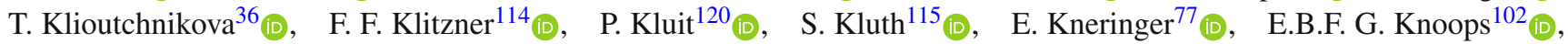




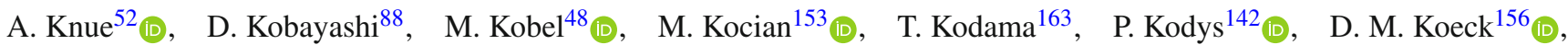

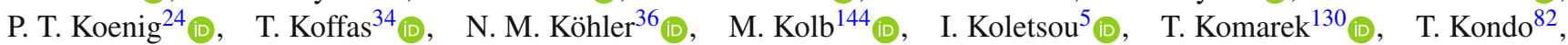
K. Köneke $e^{52}$ (D), A.X. Y. Kong ${ }^{1}$ (D)， A. C. König ${ }^{119}$ (D), T. Kono ${ }^{126}$ (D), V. Konstantinides ${ }^{95}, \quad$ N. Konstantinidis ${ }^{95}$ (D), B. Konya ${ }^{97}$ (D), R. Kopeliansky ${ }^{66}$ (D), S. Koperny ${ }^{84 a} a_{(D)}, \quad$ K. Korcyl ${ }^{85}$ (D), K. Kordas ${ }^{162}$ (D), G. Koren ${ }^{161}$, A. Korn ${ }^{95}$ (D), I. Korolkov ${ }^{14}$ (D), E. V. Korolkova ${ }^{149}$, N. Korotkova ${ }^{113}$ (D), O. Kortner ${ }^{115}$ (D), S. Kortner ${ }^{115}$ (D), V. V. Kostyukhin ${ }^{149,166}$ (D), A. Kotsokechagia ${ }^{65}$ (D), A. Kotwal $^{49}$ (D), A. Koulouris ${ }^{10}$ (D), A. Kourkoumeli-Charalampidi ${ }^{71 a, 71 b}$ (D), C. Kourkoumelis ${ }^{9}$ (D), E. Kourlitis ${ }^{6}$ (D) V. Kouskoura ${ }^{29}$ (D), R. Kowalewski ${ }^{176}$ (D) W. Kozanecki ${ }^{101}$ (D), A. S. Kozhin ${ }^{123}$ (D), V. A. Kramarenko ${ }^{113}$ (D),

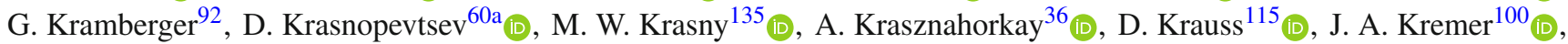

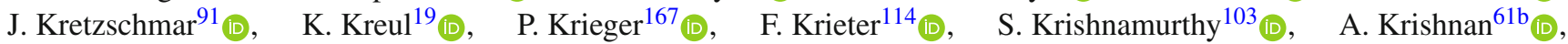

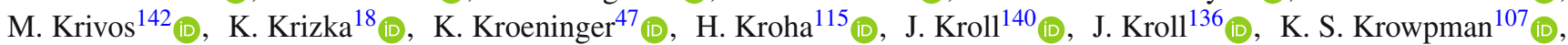

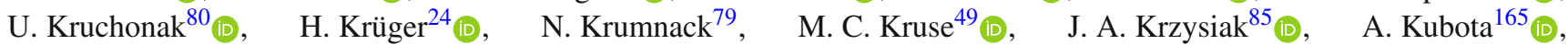
O. Kuchinskaia ${ }^{16} 6_{(D)}$, S. Kuday ${ }^{4 b}$ (D) , D. Kuechler ${ }^{46}$ (D), J. T. Kuechler ${ }^{46}$ (D), S. Kuehn ${ }^{36}$ (D), T. Kuhl ${ }^{46}$ (D), V. Kukhtin ${ }^{80}$ (D), Y. Kulchitsky ${ }^{108, a e}$ (D), S. Kuleshov ${ }^{146 b}{ }_{(\mathbb{D})}$, Y. P. Kulinich ${ }^{173}$, M. Kuna ${ }^{58}$ (D), A. Kupco ${ }^{140}$ (D), T. Kupfer ${ }^{47}$, O. Kuprash ${ }^{52}$ (D), H. Kurashige ${ }^{83}$ (D) L. L. Kurchaninov ${ }^{168 a}$ (D), Y. A. Kurochkin ${ }^{108}$ (D) A. Kurova ${ }^{112}$ (D), M. G. Kurth ${ }^{15 a}, 15 d$, E. S. Kuwertz ${ }^{36}$ (D), M. Kuze ${ }^{165}$ (D), A. K. Kvam ${ }^{148}$ (D), J. Kvita ${ }^{130}$ (D), T. Kwan ${ }^{104}$ (D), C. Lacasta ${ }^{174}$ (D), F. Lacava ${ }^{73 a, 73 b}$ (D), D.P. J. Lack ${ }^{101}$ (D),

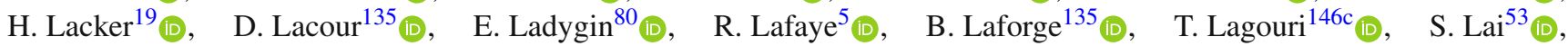

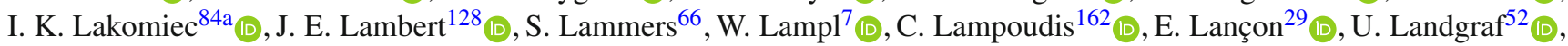
M.P. J. Landon ${ }^{93}$ (D), V. S. Lang ${ }^{52}$ (i), J. C. Lange $e^{53}$ (i), R. J. Langenberg ${ }^{103}$ (I), A. J. Lankford ${ }^{171}$ (i), F. Lanni ${ }^{29}$ (D),

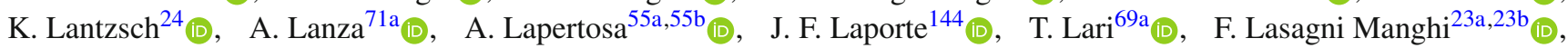

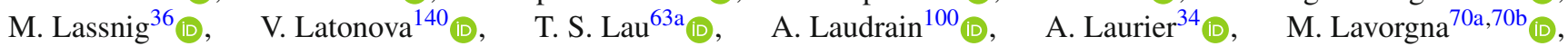

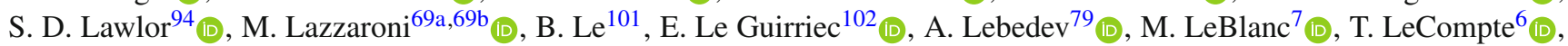

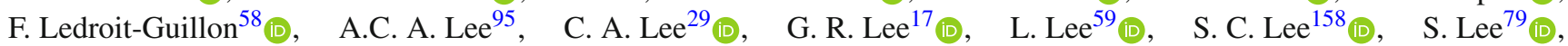

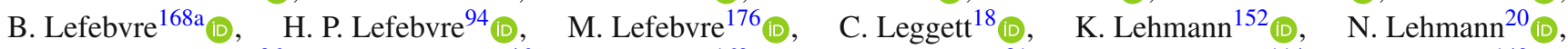

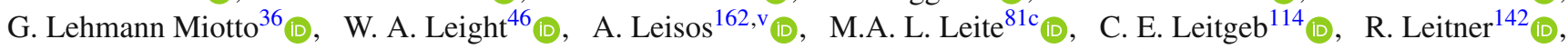
K. J. C. Leney ${ }^{42}$ (D), T. Lenz ${ }^{24}$ (D), S. Leone ${ }^{72 a}$ (D), C. Leonidopoulos ${ }^{50}$ (D), A. Leopold ${ }^{135}$ (D) C. Leroy ${ }^{110}$ (D) R. Les ${ }^{107}$ (D),

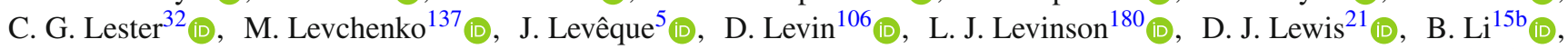

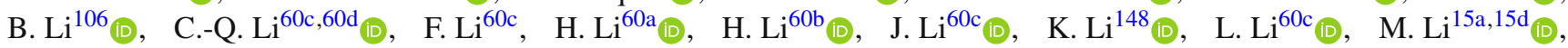

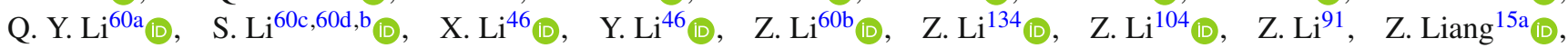

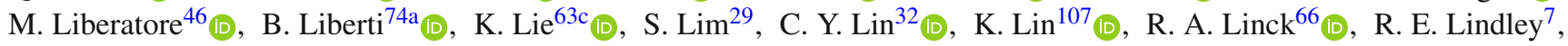

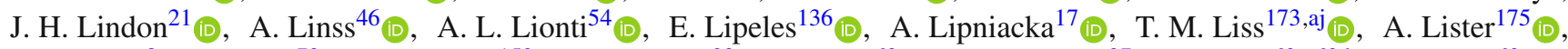

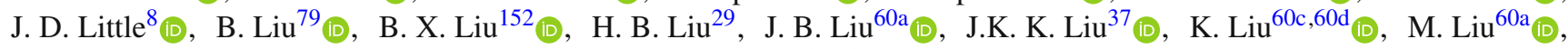

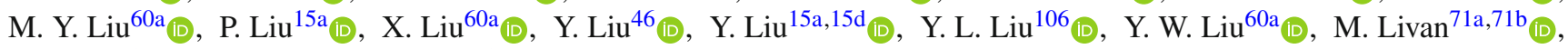

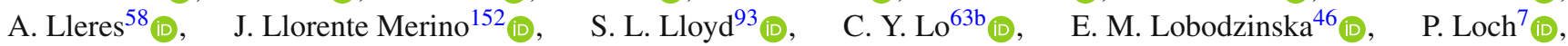

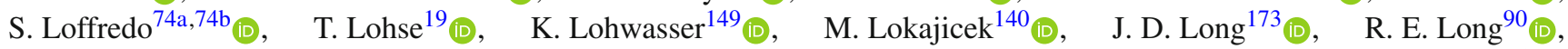
I. Longarini ${ }^{73 a}, 73 b$ (D), L. Longo ${ }^{36}$, I. Lopez Paz ${ }^{101}$, A. Lopez Solis ${ }^{149}$ (D), J. Lorenz ${ }^{114}$ (D), N. Lorenzo Martinez ${ }^{5}$ (1),

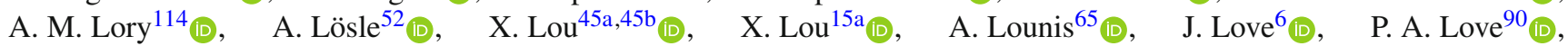

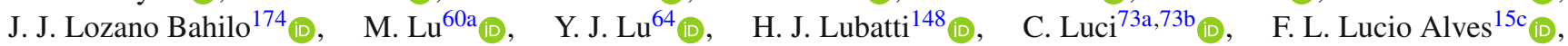

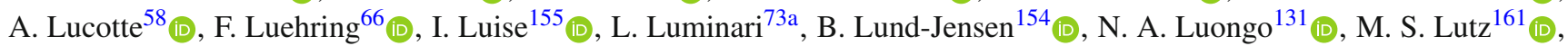

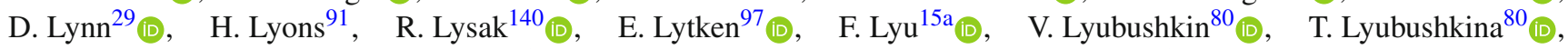

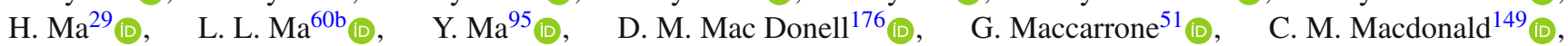
J. C. MacDonald ${ }^{149}$ (D), J. Machado Miguens ${ }^{136}{ }^{\mathbb{D}}$, R. $\operatorname{Madar}^{38}$ (D), W. F. Mader ${ }^{48}$ (D) M. Madugoda Ralalage Don ${ }^{129}$ (D),

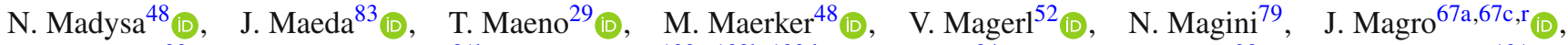

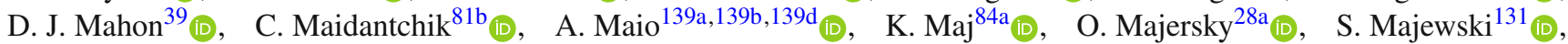
Y. Makida ${ }^{82}$, N. Makovec ${ }^{65}$ (), B. Malaescu ${ }^{135}$ (D), Pa. Malecki ${ }^{85}$ (D), V. P. Maleev ${ }^{137}$ (D), F. Malek ${ }^{58}$ (D), D. Malito ${ }^{41 \mathrm{a}, 41 \mathrm{~b}}$ (D), U. Mallik ${ }^{78}$ (D) C. Malone ${ }^{32}$ (I), S. Maltezos ${ }^{10}$, S. Malyukov ${ }^{80}$, J. Mamuzic ${ }^{174}$ (D), G. Mancini ${ }^{51}$ (D), J. P. Mandalia ${ }^{93}$ (D), I. Mandić92 (D), L. Manhaes de Andrade Filho ${ }^{81 a}$ (D), I. M. Maniatis ${ }^{162}$ (D) J. Manjarres $\operatorname{Ramos}^{48}$ (D), K. H. Mankinen ${ }^{97}$ (D), A. Mann ${ }^{114}$ (D), A. Manousos ${ }^{77}$ (D), $\quad$ B. Mansoulie ${ }^{144}$ (D), I. Manthos ${ }^{162}$ (1), S. Manzoni ${ }^{120}$ (D), A. Marantis ${ }^{162}$ (D),

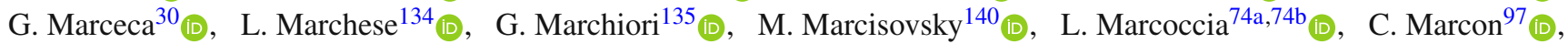
M. Marjanovic ${ }^{128}$ (D) Z. Marshall ${ }^{18}$ (D), M.U. F. Martensson ${ }^{172}$ (D), S. Marti-Garcia ${ }^{174}$ (D), C. B. Martin ${ }^{127}$ (D), T. A. Martin ${ }^{178}$ (D), V. J. Martin ${ }^{50}$ (D) $\quad$ B. Martin dit Latour ${ }^{17}$ (D), L. Martinelli ${ }^{75 a, 75 b}$ (D) $\quad$ M. Martinez ${ }^{14, w}$ (D),$\quad$ P. Martinez Agullo ${ }^{174}$ (D), V. I. Martinez Outschoorn ${ }^{103}$ (D), S. Martin-Haugh ${ }^{143}$ (D), V. S. Martoiu ${ }^{27 b}$ (D), A. C. Martyniuk ${ }^{95}$ (D), A. Marzin ${ }^{36}$ (D),

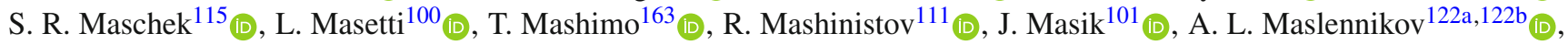

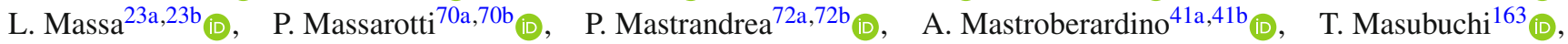




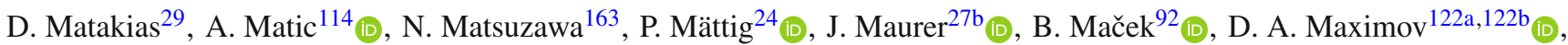

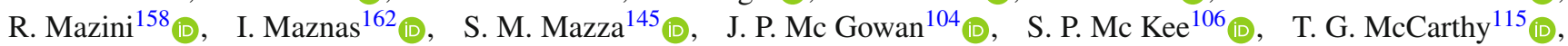

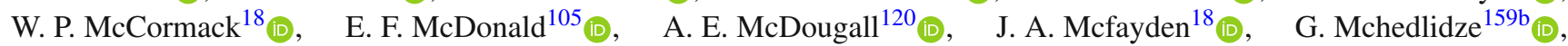

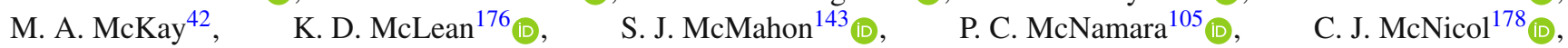

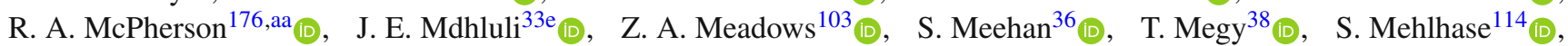

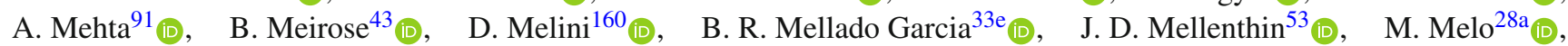
F. Meloni ${ }^{46}(\mathbb{D})$ A. Melzer $^{24}$ (D), E. D. Mendes Gouveia ${ }^{139 a, 139 e}{ }_{(\mathbb{D})}$, A. M. Mendes Jacques Da Costa ${ }^{21}$ (D), H. Y. Meng ${ }^{167}$,

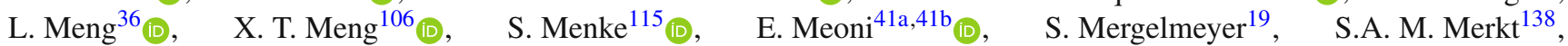

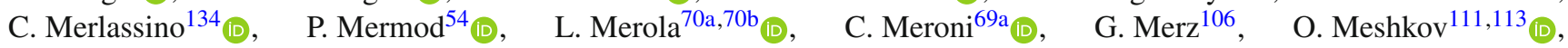

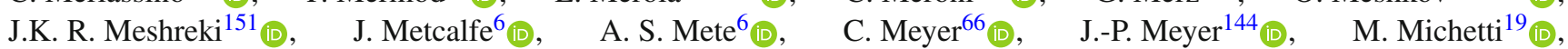
R. P. Middleton ${ }^{143}$ (D), L. Mijović ${ }^{50}$ (D), G. Mikenberg ${ }^{180}$ (D), M. Mikestikova ${ }^{140}$ (D), M. Mikuž $\check{z}^{92}$ (D), H. Mildner ${ }^{149}$ (D),

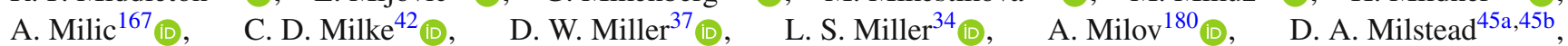

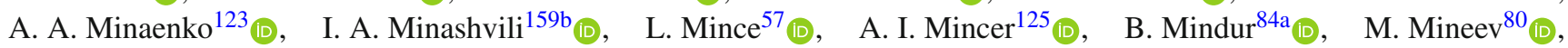
Y. Minegishi ${ }^{163}$, Y. Mino ${ }^{86}$ (D), L. M. Mir $^{14}$ (D), M. Mironova ${ }^{134}$, T. Mitani ${ }^{179}$ (D), J. Mitrevski ${ }^{114}$, V. A. Mitsou ${ }^{174}$ (D),

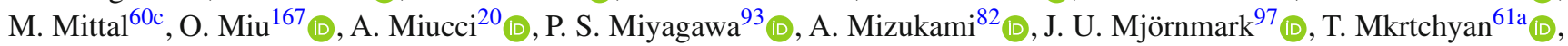

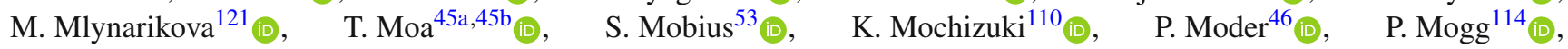
S. Mohapatra ${ }^{39}$ (D) R. Moles-Valls ${ }^{24}$ (D) K. Mönig ${ }^{46}$ (D), E. Monnier ${ }^{102}$ (D), A. Montalbano ${ }^{152}$ (D), J. Montejo Berlingen ${ }^{36}$ (D),

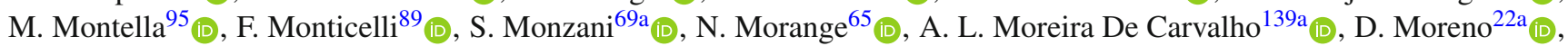
M. Moreno Llácer ${ }^{174}$ (D), C. Moreno Martinez ${ }^{14}$ (D), P. Morettini ${ }^{55 b}{ }_{(\mathbb{C})}, \quad$ M. Morgenstern ${ }^{160}$ (D), S. Morgenstern ${ }^{48}$ (D), D. Mori ${ }^{152}$ (D) M. Morii ${ }^{59}$ (D) M. Morinaga ${ }^{179}$ (D), V. Morisbak ${ }^{133}$ (D) A. K. Morley ${ }^{36}$ (D) , G. Mornacchi ${ }^{36}$ (D) , A. P. Morris ${ }^{95}$ (D),

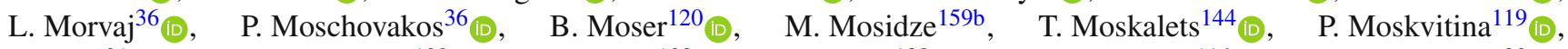
J. Moss ${ }^{31, n}$ (D), E. J. W. Moyse ${ }^{103}$ (D), S. Muanza ${ }^{102}$ (D), J. Mueller ${ }^{138}$ (D), R.S. P. Mueller ${ }^{114}$, D. Muenstermann ${ }^{90} \mathbb{C}_{\text {(D) }}$,

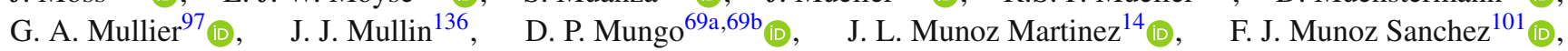
P. Murin ${ }^{28 b}$ (D), W. J. Murray ${ }^{143,178}$ (D), A. Murrone ${ }^{69 a, 69 b}$ (D), J. M. Muse ${ }^{128}$ (D), M. Muškinja ${ }^{18}$ (D), C. Mwewa ${ }^{33 a}$ (D),

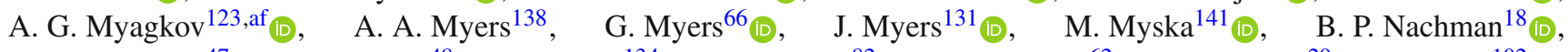
O. Nackenhorst ${ }^{47}$ (D), A.Nag Nag ${ }^{48}$ (D), K. Nagai ${ }^{134}$ (D), K. Nagano ${ }^{82}$ (D), Y. Nagasaka ${ }^{62}$ (D), J. L. Nagle ${ }^{29}$ (D), E. Nagy ${ }^{102}$ (D),

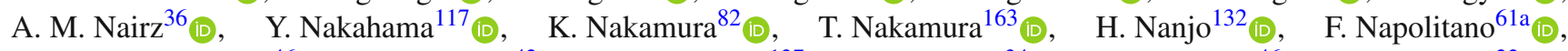

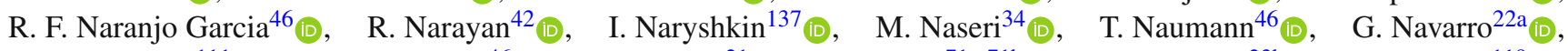

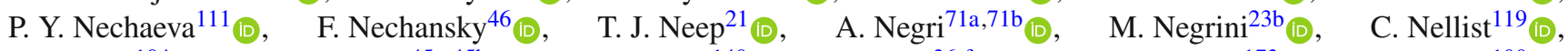

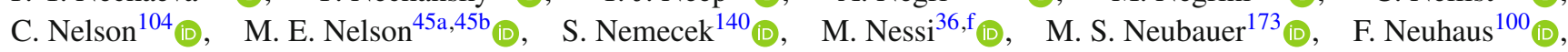

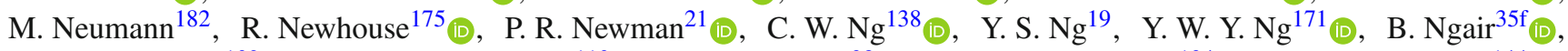

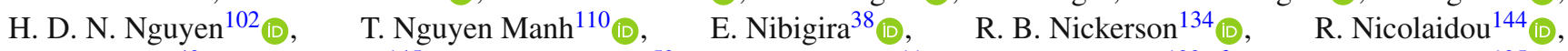

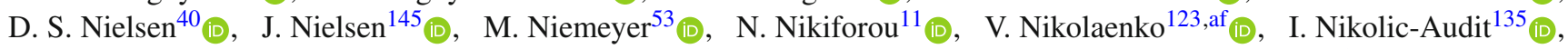
K. Nikolopoulos ${ }^{21}$ (D), P. Nilsson ${ }^{29}$ (D) H. R. Nindhito ${ }^{54}$ (D), A. Nisati ${ }^{73 \mathrm{a}}$ (i), N. Nishu ${ }^{60 \mathrm{c}}$ (D) R. Nisius ${ }^{115}$ (D), I. Nitsche ${ }^{47}$, T. Nitta ${ }^{179}$ (D), T. Nobe ${ }^{163}$ (D), D. L. Noel $^{32}$ (D), Y. Noguchi ${ }^{86}$ (D), I. Nomidis ${ }^{135}$ (D), M. A. Nomura ${ }^{29}$, M. Nordberg ${ }^{36}$,

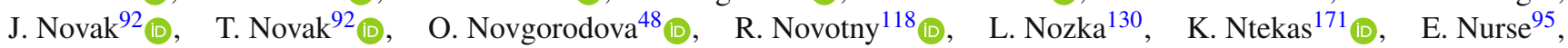

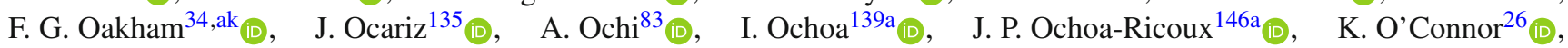

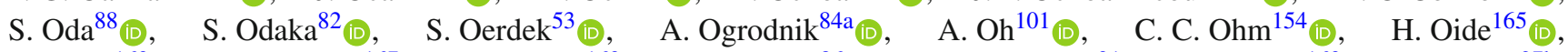
R. Oishi ${ }^{163}$ (D), M. L. Ojeda ${ }^{167}$ (I), H. Okawa $^{169}$ (D), Y. Okazaki ${ }^{86}$ (D) M. W. O’ Keefe $^{91}$, Y. Okumura ${ }^{163}$ (D), A. Olariu ${ }^{27 b}$,

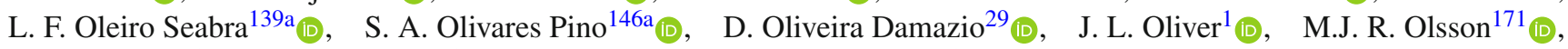
A. Olszewski ${ }^{85}$ (D), J. Olszowska ${ }^{85}$ (D), Ö. O. Öncel ${ }^{24}$ (D), D. C. O’Neil ${ }^{152}$ (D), A. P. O’neill ${ }^{134}$ (D), A. Onofre ${ }^{139 a, 139 e}$ (D), P.U. E. Onyisi ${ }^{11}$ (D) H. Oppen ${ }^{133}$, R. G. Oreamuno Madriz ${ }^{121}$, M. J. Oreglia ${ }^{37} \mathbb{D}$, G. E. Orellana ${ }^{89}$ (D) D. Orestano ${ }^{75 a}, 75 b$ (D),

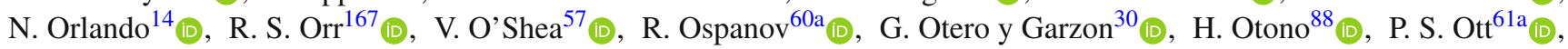

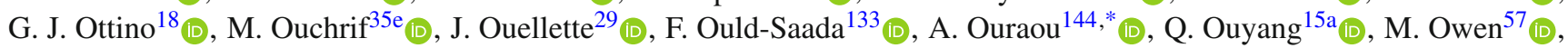

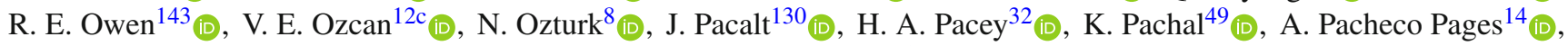

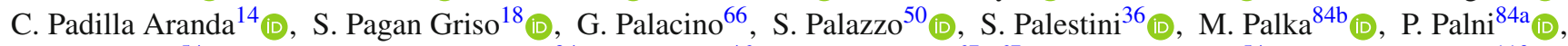

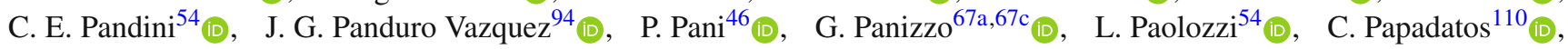

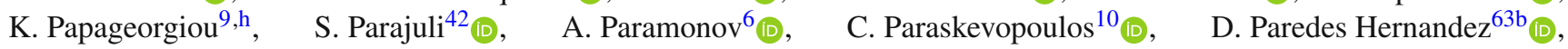
S. R. Paredes Saenz ${ }^{134}$ (I),$\quad$ B. Parida ${ }^{180}$ (D), T. H. Park ${ }^{167}$ (D), A. J. Parker ${ }^{31}$ (D), M. A. Parker ${ }^{32}$ (D), F. Parodi ${ }^{55 a, 55 b}$ (D), E. W. Parrish ${ }^{121}$ (D) J. A. Parsons ${ }^{39}$ (D) U. Parzefall ${ }^{52}$ (D) L. Pascual Dominguez ${ }^{135}$ (D) , V. R. Pascuzzi ${ }^{18}$ (D) J.M. P. Pasner ${ }^{145}$ (D),

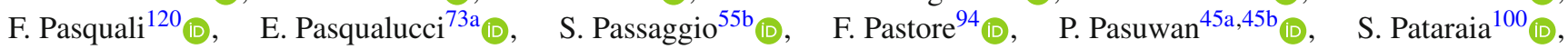

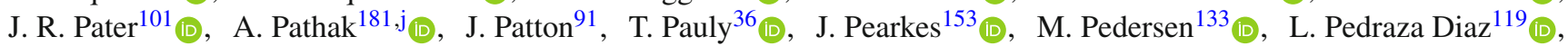

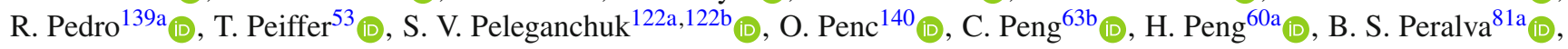




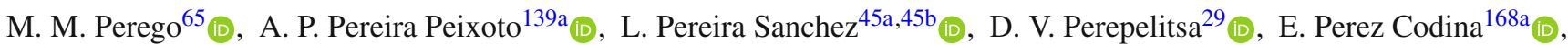

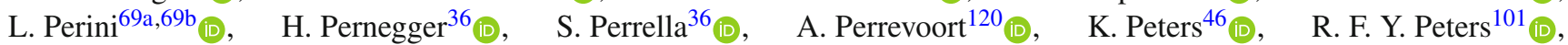

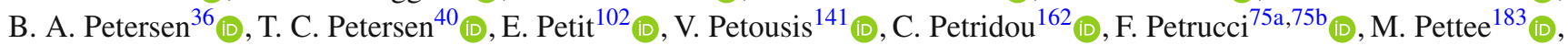

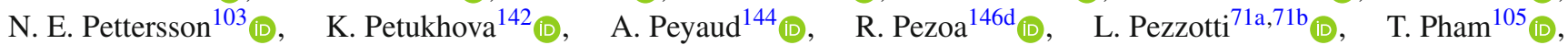

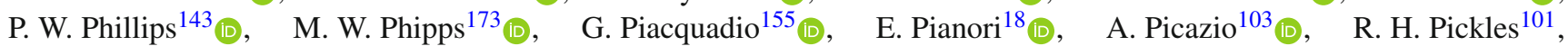

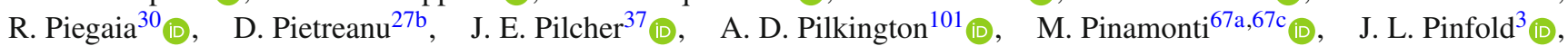
C. Pitman Donaldson ${ }^{95}$ ， M. Pitt ${ }^{161}{ }_{(D)}, \quad$ L. Pizzimento ${ }^{74 a, 74 b}{ }_{(D)}, \quad$ A. Pizzini ${ }^{120}{ }_{(D)}, \quad$ M.-A. Pleier ${ }^{29}$ (D), V. Plesanovs ${ }^{52}$, V. Pleskot ${ }^{142}$ (D), E. Plotnikova ${ }^{80}, \quad$ P. Podberezko ${ }^{122 a, 122 b}$ (D) $_{1}, \quad$ R. Poettgen ${ }^{97}$ (D), R. Poggi ${ }^{54}$ (D), L. Poggioli $^{135}$ (D),

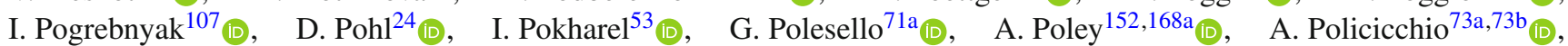
R. Polifka ${ }^{142}$ (D), A. Polini ${ }^{23 b}{ }_{(D)}$, C. S. Pollard ${ }^{46}$, V. Polychronakos ${ }^{29}$ (D), D. Ponomarenko ${ }^{112}$ (D), L. Pontecorvo ${ }^{36}$ (D),

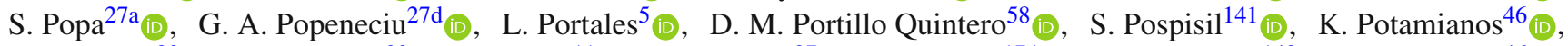

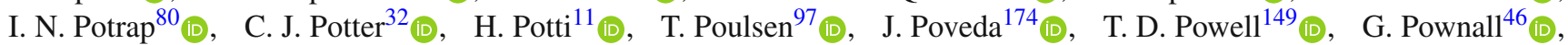
M. E. Pozo Astigarraga ${ }^{36}$ (D) A. Prades Ibanez ${ }^{174}$ (D), P. Pralavorio ${ }^{102}$ (D), M. M. Prapa ${ }^{44}$ (D) S. Prell ${ }^{79}$ (D), D. Price ${ }^{101}$ (D),

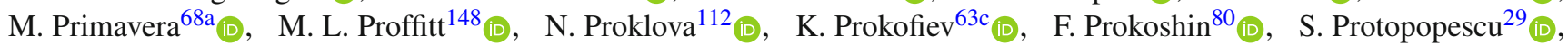

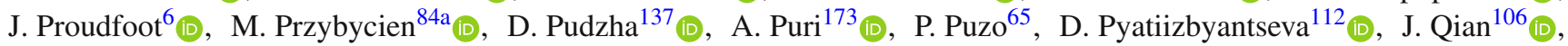
Y. Qin ${ }^{101}$ (D), A. Quadt ${ }^{53}$ (D), M. Queitsch-Maitland ${ }^{36}$ (D), G. Rabanal Bolanos ${ }^{59}$ (D), M. Racko ${ }^{28 a}$, F. Ragusa ${ }^{69 a}, 69 b$ (D),

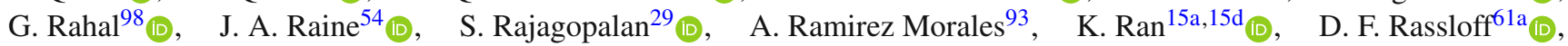

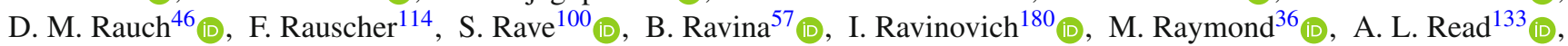

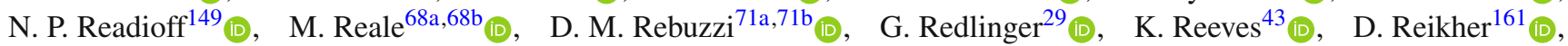

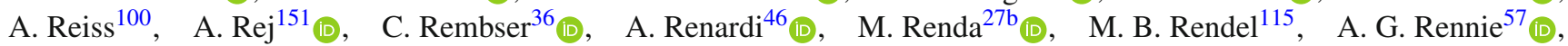

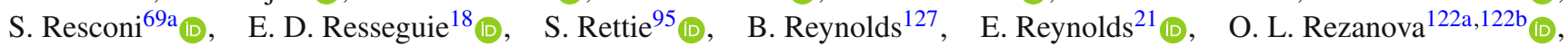

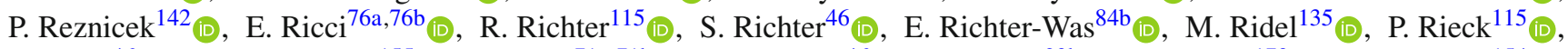

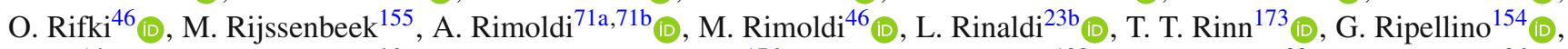
I. Riu ${ }^{14}$ (D), P. Rivadeneira ${ }^{46}$ (D), J. C. Rivera Vergara ${ }^{176}$ (D), F. Rizatdinova ${ }^{129}$ (D), E. Rizvi ${ }^{93}$ (D), C. Rizzi ${ }^{36}$ (D),

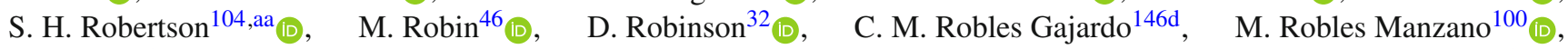

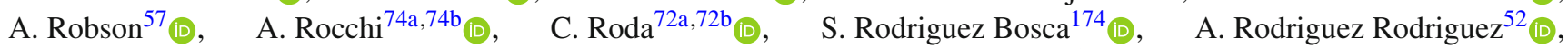
A. M. Rodríguez Vera ${ }^{168 b}$ (D) S. Roe ${ }^{36}$, J. Roggel ${ }^{182}$ (D), O. Røhne ${ }^{133}$ (D), R. Röhrig ${ }^{115}$ (D), R. A. Rojas ${ }^{146 d}$ (D) B. Roland ${ }^{52}$ (D), C.P. A. Roland ${ }^{66}$ (D), J. Roloff ${ }^{29}$ (D), A. Romaniouk ${ }^{112}$ (D), M. Romano ${ }^{23 a, 23 b}{ }_{(\mathbb{C})}, \quad$ N. Rompotis ${ }^{91}$ (D), M. Ronzani ${ }^{125}$ (D),

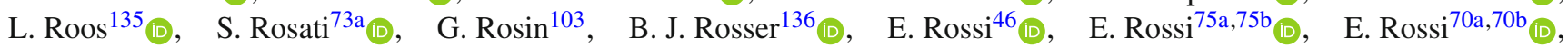

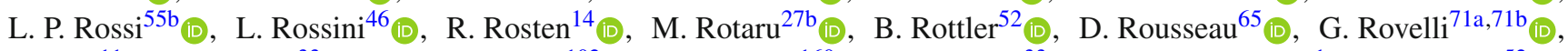

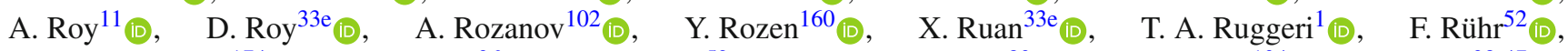
A. Ruiz-Martinez ${ }^{174}$ (D), A. Rummler ${ }^{36}$ (D) Z. Rurikova ${ }^{52}$ (D), N. A. Rusakovich ${ }^{80}$ (D) H. L. Russel1 ${ }^{104}$ (I) , L. Rustige ${ }^{38,47}$ (D),

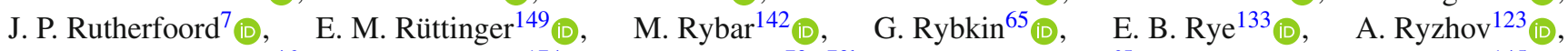

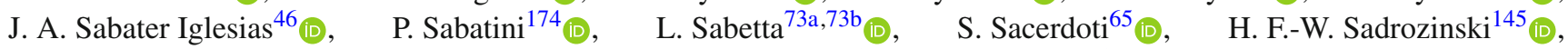

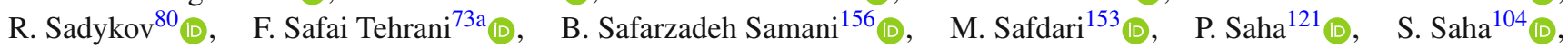

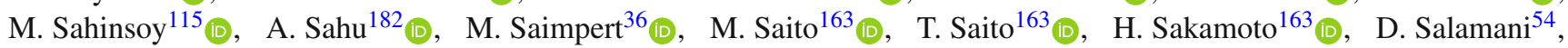

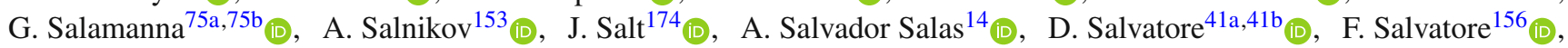

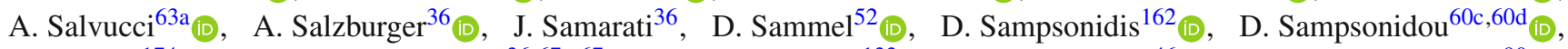
J. Sánchez ${ }^{174}$ (D) $\quad$ A. Sanchez Pineda ${ }^{36,67 a, 67 c}$ (D), H. Sandaker ${ }^{133}$ (D), C. O. $\operatorname{Sander}^{46}$ (D), I. G. Sanderswood ${ }^{90}$ (D),

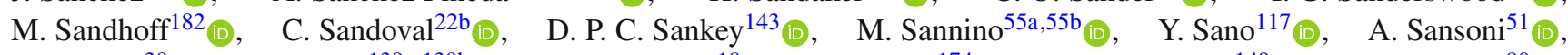

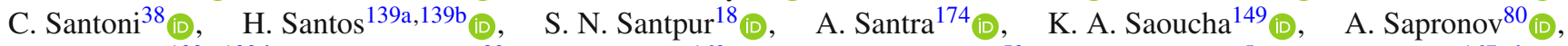

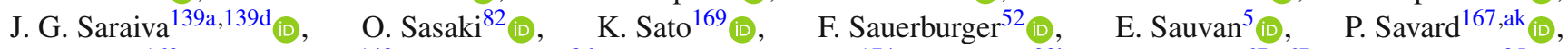

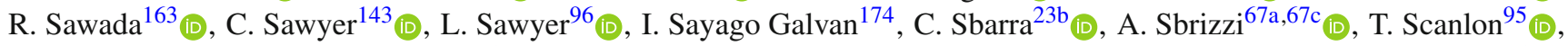

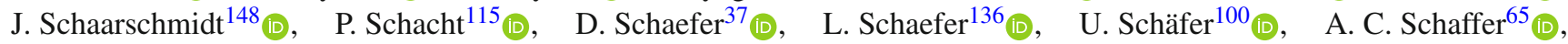
D. Schaile ${ }^{114}$ (D), R. D. Schamberger ${ }^{155}$ (D), E. Schanet ${ }^{11^{1}}$ (D), C. Scharf ${ }^{19}$ (D), N. Scharmberg ${ }^{101}$ (D), V. A. Schegelsky ${ }^{137}$ (D),

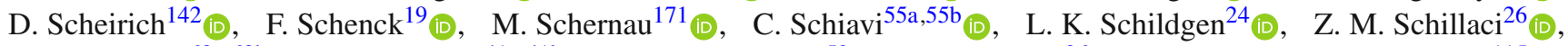

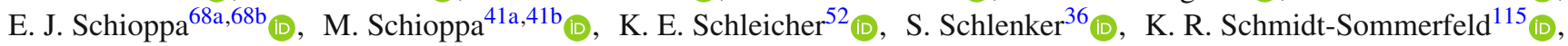

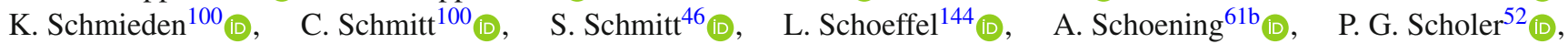
E. Schopf ${ }^{134}$ (D), M. Schott ${ }^{100}$ (D), J.F. P. Schouwenberg ${ }^{19}$ (D), J. Schovancova ${ }^{36}$ (D), S. Schramm ${ }^{54}$ (D), F. Schroeder ${ }^{182}$ (D), A. Schulte ${ }^{100}\left(\right.$ D,$\quad$ H-C. Schultz-Coulon ${ }^{61 \mathrm{a}}\left(\mathbb{D}, \quad\right.$ M. Schumacher ${ }^{52}$ (D), B. A. Schumm ${ }^{145}$ (D), Ph. Schune ${ }^{144}$ (D), A. Schwartzman ${ }^{153}$ (D) T. A. Schwarz ${ }^{106}$ (D), Ph. Schwemling ${ }^{144}$ (D), R. Schwienhorst ${ }^{107}$ (D), A. Sciandra ${ }^{145}$ (D), G. Sciolla ${ }^{26}$ (D),

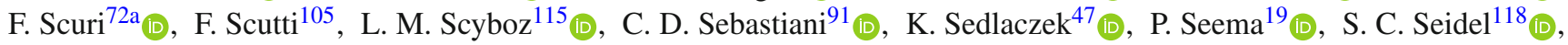

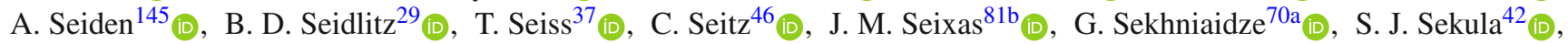




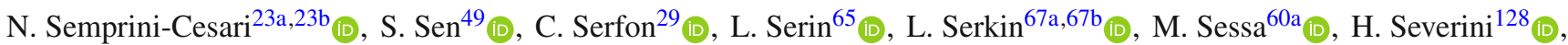

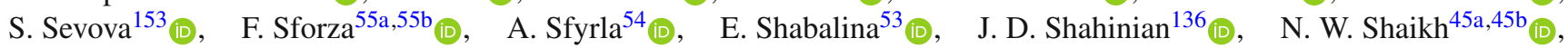

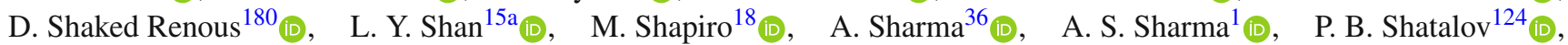

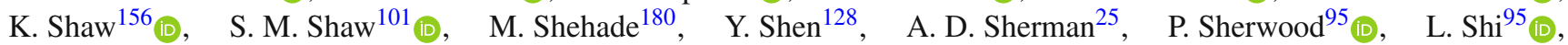
C. O. Shimmin ${ }^{183}$ (D), Y. Shimogama ${ }^{179}$ (D), M. Shimojima ${ }^{116}$ (D), J. D. Shinner ${ }^{94}$ (D), I.P. J. Shipsey ${ }^{134}$ (D), S. Shirabe ${ }^{165}{ }^{(D)}$,

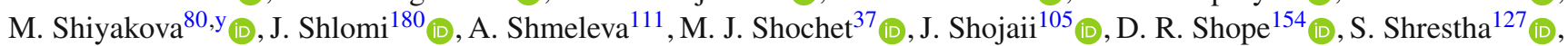

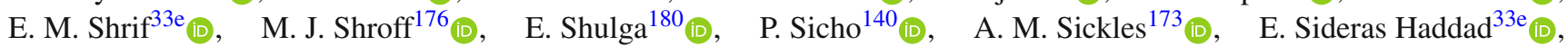

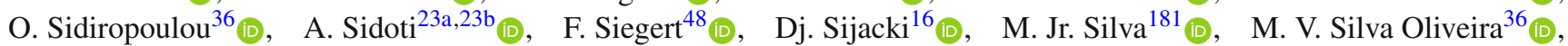

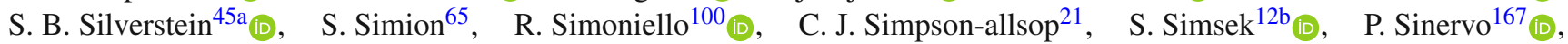
V. Sinetckii ${ }^{113}$ (D) S. Singh ${ }^{152}$ (D), S. Sinha ${ }^{33 e^{(D)}}$, M. Sioli ${ }^{23 a, 23 b}$ (D), I. Siral ${ }^{131}$ (D), S. Yu. Sivoklokov ${ }^{113}$ (D), J. Sjölin ${ }^{45 a, 45 b}$ (D), A. Skaf ${ }^{53}$ (D) $\quad$ E. Skorda ${ }^{97}$, P. Skubic ${ }^{128}$ (D), M. Slawinska ${ }^{85}$ (D), K. Sliwa ${ }^{170}$ (D) V. Smakhtin ${ }^{180}, \quad$ B. H. Smart ${ }^{143}$ (D),

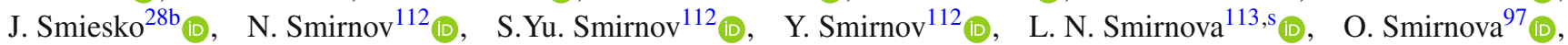

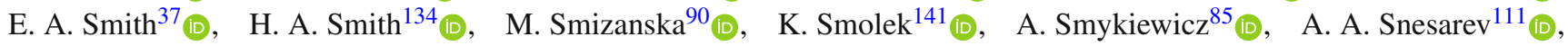
H. L. Snoek ${ }^{120}$ (D), I. M. Snyder ${ }^{131}$ (D), S. Snyder ${ }^{29}$ (D), R. Sobie ${ }^{176, a a}$ (D), A. Soffer ${ }^{161}$ (D) A. Søgaard ${ }^{50}$ (D) F. Sohns ${ }^{53}$ (D),

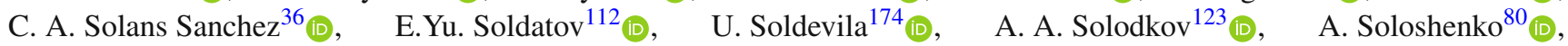

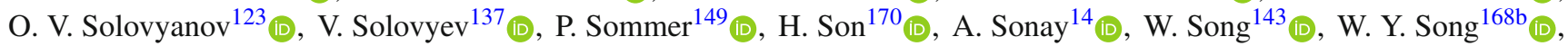

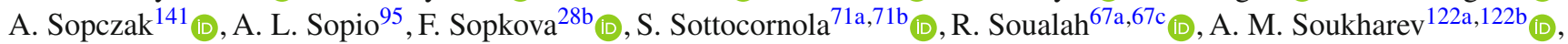

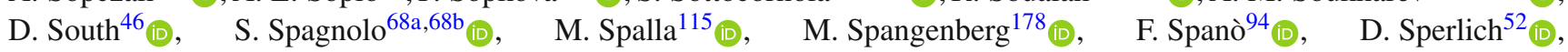

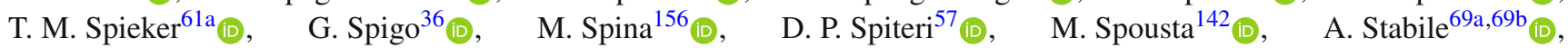

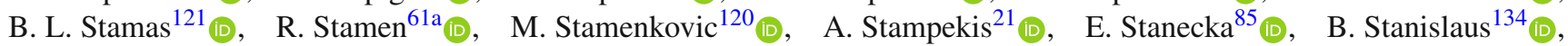

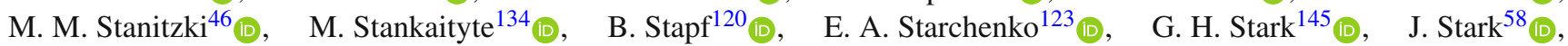

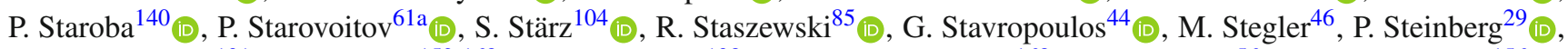

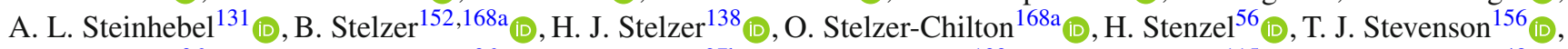

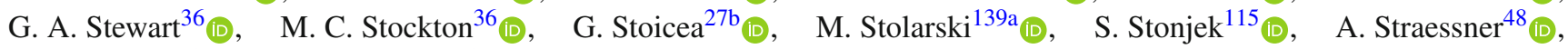
J. Strandberg ${ }^{154}$ (D), S. Strandberg ${ }^{45 a, 45 b}$ (D), M. Strauss ${ }^{128}$ (D), T. Strebler ${ }^{102}$ (I),$\quad$ P. Strizenec ${ }^{28 b}$ (D), R. Ströhmer ${ }^{177}$ (D),

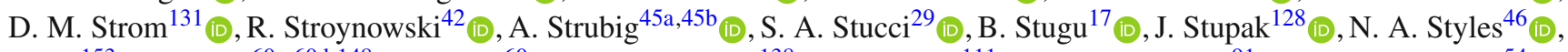

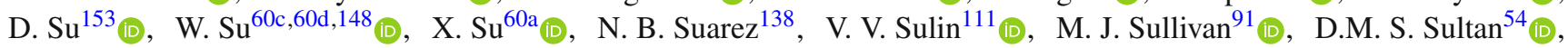

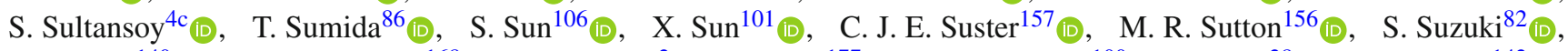

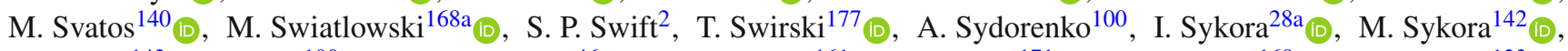
T. Sykora ${ }^{142}$ (D), D. Ta ${ }^{100}$ (D), K. Tackmann ${ }^{46, x_{(D)}, \quad J . ~ T a e n z e r ~}{ }^{161}, \quad$ A. Taffard ${ }^{171}$ (D), R. Tafirout ${ }^{168 a}$ (D), E. Tagiev ${ }^{123}$ (D), R. H. M. Taibah ${ }^{135}$ (D), R. Takashima ${ }^{87}$, K. Takeda ${ }^{83}$ (D) T. Takeshita ${ }^{150}$ (D), E. P. Takeva ${ }^{50}$ (D), Y. Takubo ${ }^{82}$ (D) M. Talby ${ }^{102}$ (D), A. A. Talyshev ${ }^{122 a, 122 b}$ (D), K. C. Tam ${ }^{63 b}, \quad$ N. M. Tamir ${ }^{161}, \quad$ J. Tanaka ${ }^{163}$ (D), R. Tanaka ${ }^{65}$ (D), S. Tapia Araya ${ }^{173}$ (D),

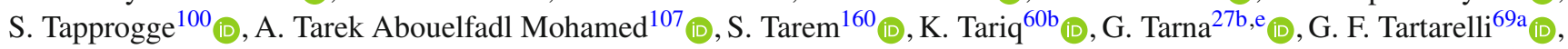

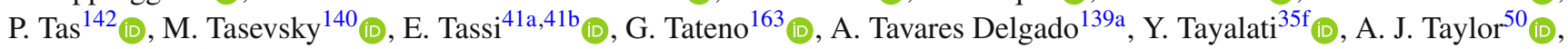

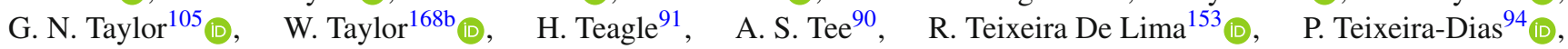

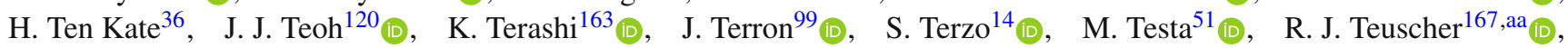

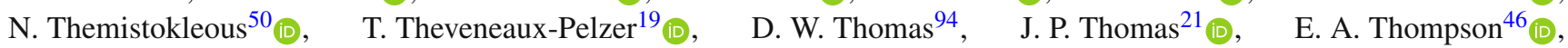

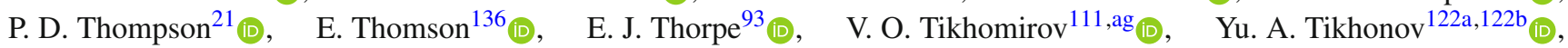

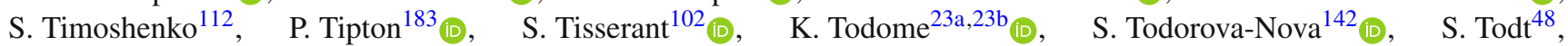
J. Tojo ${ }^{88}$ (D), S. Tokár ${ }^{28}$ (D), K. Tokushuku ${ }^{82}$ (D), E. Tolley ${ }^{127}$ (D), R. Tombs ${ }^{32}$ (D), K. G. Tomiwa ${ }^{33 e_{\text {(D) }}, \text { M. Tomoto }}{ }^{82,117}$ (D),

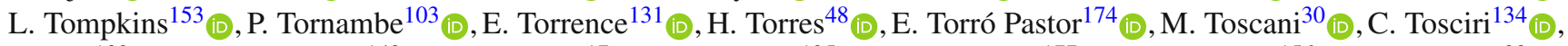
J. Toth ${ }^{102, z}$ (D), D. R. Tovey ${ }^{149}$ (D), A. Traeet ${ }^{17}$, C. J. Treado ${ }^{125}$ (D), T. Trefzger ${ }^{177}$ (D), F. Tresoldi ${ }^{156}$ (I) A. Tricoli ${ }^{29}$ (D),

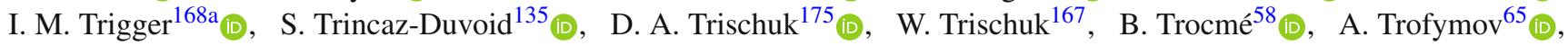

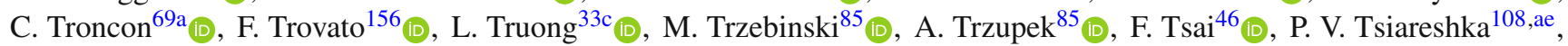
A. Tsirigotis ${ }^{162, \mathrm{v}}$ (D) , V. Tsiskaridze ${ }^{155}$ (D), E. G. Tskhadadze ${ }^{159 a}$, M. Tsopoulou ${ }^{162}$ (D) I. I. Tsukerman ${ }^{124}$ (D), V. Tsulaia ${ }^{18}$ (D),

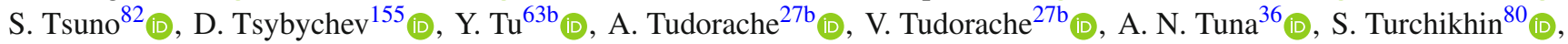
D. Turgeman ${ }^{180}$ (D), I. Turk Cakir ${ }^{4 b, t}$, R. J. Turner ${ }^{21}$, R. Turra ${ }^{69}$ (D) P. M. Tuts ${ }^{39}$ (D) S. Tzamarias ${ }^{162}$, E. Tzovara ${ }^{100}$ DD $_{\text {, }}$

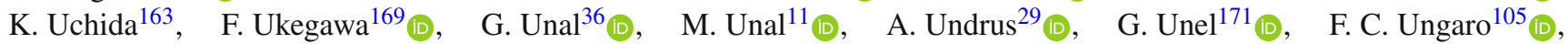

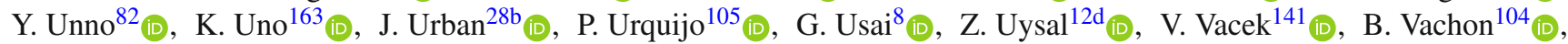

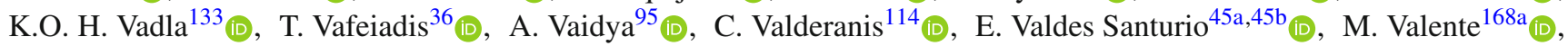

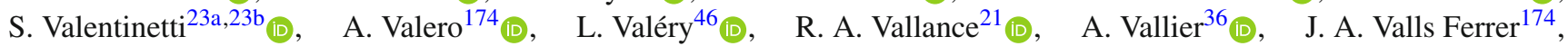

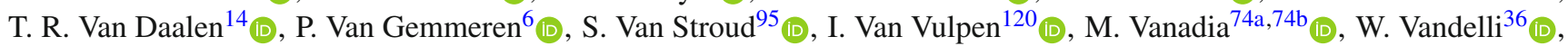

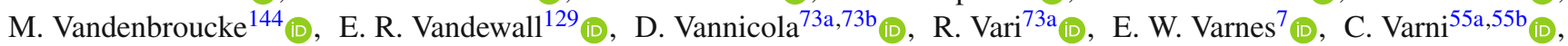




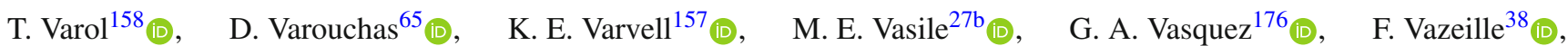
D. Vazquez Furelos ${ }^{14}$ (D), T. Vazquez Schroeder ${ }^{36}$ (D) J. Veatch ${ }^{53}$ (D), V. Vecchio ${ }^{101}$ (D), M. J. Veen ${ }^{120}$ (D), L. M. Veloce ${ }^{167}$ (D),

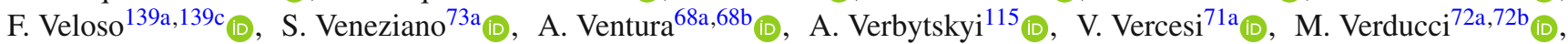
C. M. Vergel Infante ${ }^{79}$, C. Vergis ${ }^{24}$ (D), W. Verkerke ${ }^{120}$ (D), A. T. Vermeulen ${ }^{120}$ (D), J. C. Vermeulen ${ }^{120}$ (D), C. Vernieri ${ }^{153}$ (D),

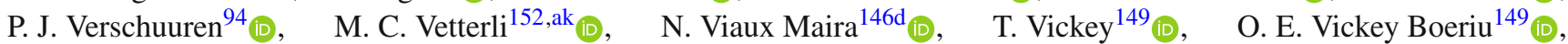

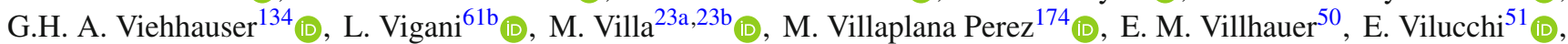

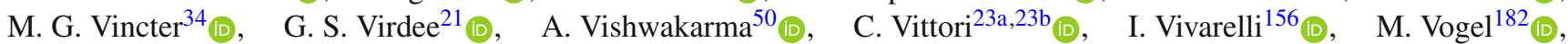

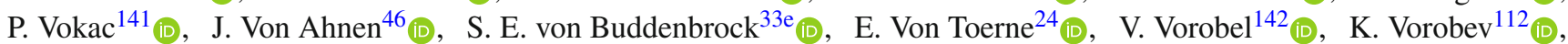

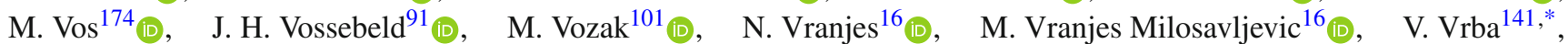
M. Vreeswijk ${ }^{120}$ (D), N. K. Vu ${ }^{102}$ (D), R. Vuillermet ${ }^{36}$ (D), I. Vukotic ${ }^{37}$ (D), S. Wada ${ }^{169}$ (D), P. Wagner ${ }^{24}$ (D), W. Wagner ${ }^{182}$ (D), J. Wagner-Kuhr ${ }^{114}$ (D), S. Wahdan ${ }^{182}$ (D), H. Wahlberg ${ }^{89}$ (D), R. Wakasa ${ }^{169}$ (D), V. M. Walbrecht ${ }^{115}$ (D), J. Walder ${ }^{143}$ (D), R. Walker ${ }^{14}$ (I),$\quad$ S. D. Walker ${ }^{94}$, W. Walkowiak ${ }^{151}$ (I), V. Wallangen ${ }^{45 a, 45 b}, \quad$ A. M. Wang ${ }^{59}$ (I), A. Z. Wang ${ }^{181}$ (D),

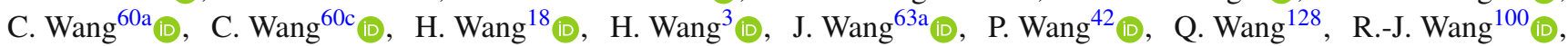

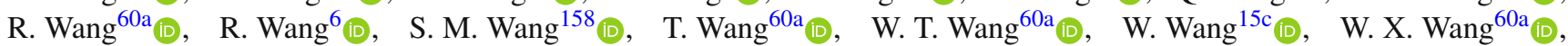

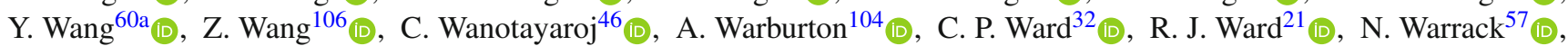
A. T. Watson ${ }^{21}$ (D) M. F. Watson ${ }^{21}$ (D), G. Watts ${ }^{148}$ (D), B. M. Waugh ${ }^{95}$ (D) A. F. Webb ${ }^{11}$ (D) C. Weber ${ }^{29}$ (D) M. S. Weber ${ }^{20}$ (D), S. A. Weber ${ }^{34}$ (D) S. M. Weber ${ }^{61 \mathrm{a}}$ (D), Y. Wei ${ }^{134}$ (D) A. R. Weidberg ${ }^{134}$ (D) , J. Weingarten ${ }^{47}$ (D) M. Weirich ${ }^{100}$ (D), C. Weiser ${ }^{52}$ (D),

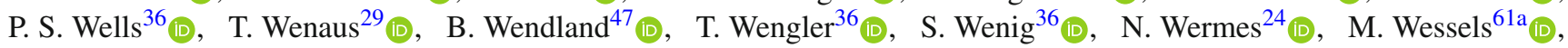

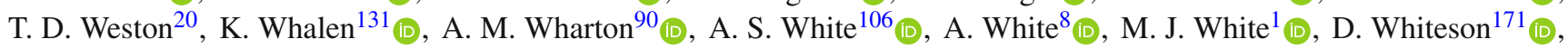

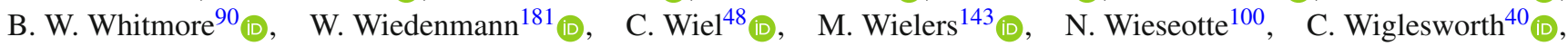
L.A. M. Wiik-Fuchs ${ }^{52}$ (D) H. G. Wilkens ${ }^{36}$ (D) L. J. Wilkins ${ }^{94}$ (i) D. M. Williams ${ }^{39}$ (1), H. H. Williams ${ }^{136}$, S. Williams ${ }^{32}$ (D), S. Willocq ${ }^{103}$ (D) P. J. Windischhofer ${ }^{134}$ (D), I. Wingerter-Seez ${ }^{5}$ (D) E. Winkels ${ }^{156}$ (D), F. Winklmeier ${ }^{131}$ (D), B. T. Winter ${ }^{52}$ (D),

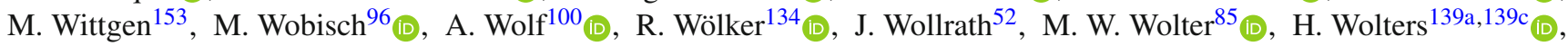

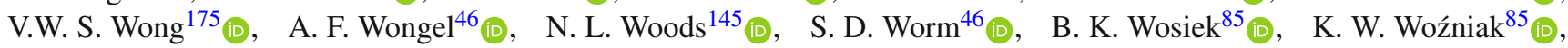

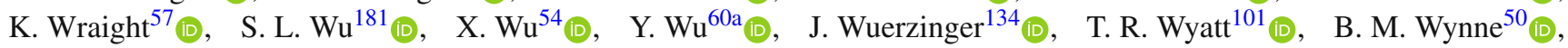

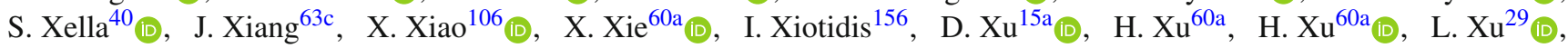

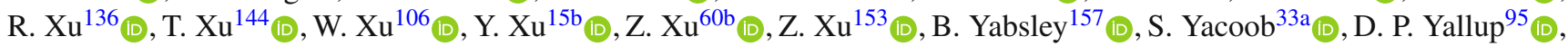
N. Yamaguchi ${ }^{88}$ (D), Y. Yamaguchi ${ }^{165}$ (D), A. Yamamoto ${ }^{82}$ (D), M. Yamatani ${ }^{163}, \quad$ T. Yamazaki ${ }^{163}$ (D), Y. Yamazaki ${ }^{83}$ (D),

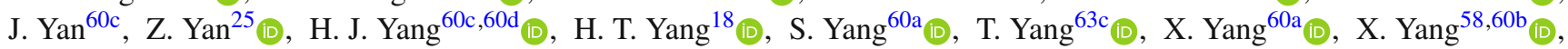

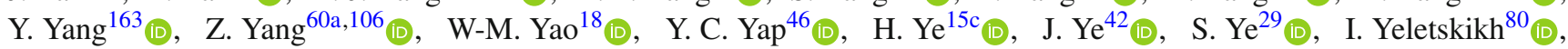
M. R. Yexley ${ }^{90}$ (D), E. Yigitbasi ${ }^{25}$ (D), P. Yin ${ }^{39}$ (D), K. Yorita ${ }^{179}$ (D), K. Yoshihara ${ }^{79}$ (D), C.J. S. Young ${ }^{36}$ (D), C. Young ${ }^{153}$ (D),

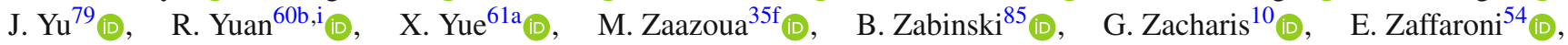

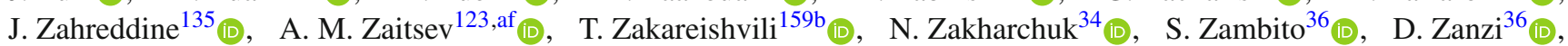

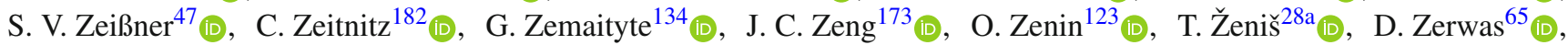

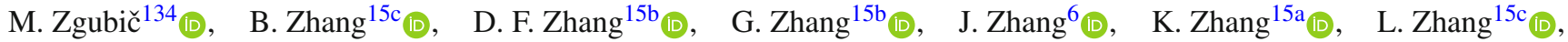

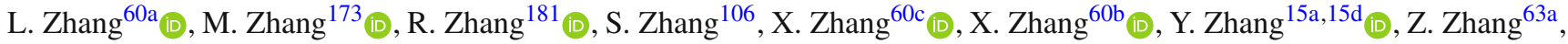

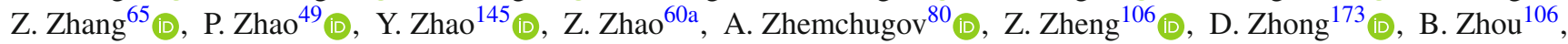

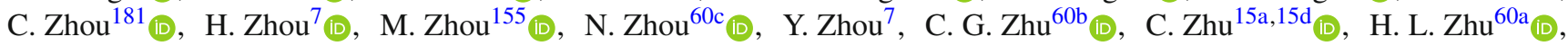

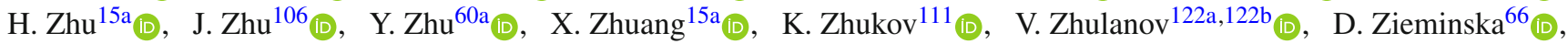

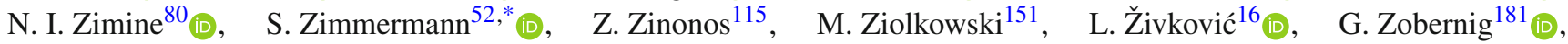
A. Zoccoli ${ }^{23 a}, 23 b$ (D) K. Zoch ${ }^{53}$ (D) , T. G. Zorbas ${ }^{149}$ (D), R. Zou ${ }^{37}$ (D) L. Zwalinski ${ }^{36}$ (D)

\footnotetext{
${ }^{1}$ Department of Physics, University of Adelaide, Adelaide, Australia

${ }^{2}$ Physics Department, SUNY Albany, Albany, NY, USA

${ }^{3}$ Department of Physics, University of Alberta, Edmonton, AB, Canada

4 (a) Department of Physics, Ankara University, Ankara, Turkey; ${ }^{(b)}$ Istanbul Aydin University, Application and Research

Center for Advanced Studies, Istanbul, Turkey; ${ }^{(c)}$ Division of Physics, TOBB University of Economics and Technology,

Ankara, Turkey

${ }^{5}$ LAPP, Université Grenoble Alpes, Université Savoie Mont Blanc, CNRS/IN2P3, Annecy, France

${ }^{6}$ High Energy Physics Division, Argonne National Laboratory, Argonne, IL, USA

${ }^{7}$ Department of Physics, University of Arizona, Tucson, AZ, USA

${ }^{8}$ Department of Physics, University of Texas at Arlington, Arlington, TX, USA

${ }^{9}$ Physics Department, National and Kapodistrian University of Athens, Athens, Greece
} 
${ }^{10}$ Physics Department, National Technical University of Athens, Zografou, Greece

${ }^{11}$ Department of Physics, University of Texas at Austin, Austin, TX, USA

12 (a) Bahcesehir University, Faculty of Engineering and Natural Sciences, Istanbul, Turkey; ${ }^{(b)}$ Istanbul Bilgi University, Faculty of Engineering and Natural Sciences, Istanbul, Turkey; ${ }^{\left({ }^{c}\right)}$ Department of Physics, Bogazici University, Istanbul, Turkey; ${ }^{(\mathrm{d})}$ Department of Physics Engineering, Gaziantep University, Gaziantep, Turkey

${ }^{13}$ Institute of Physics, Azerbaijan Academy of Sciences, Baku, Azerbaijan

${ }^{14}$ Institut de Física d'Altes Energies (IFAE), Barcelona Institute of Science and Technology, Barcelona, Spain

15 (a) Institute of High Energy Physics, Chinese Academy of Sciences, Beijing, China; ${ }^{(b)}$ Physics Department, Tsinghua University, Beijing, China; ${ }^{(c)}$ Department of Physics, Nanjing University, Nanjing, China; ${ }^{(d)}$ University of Chinese Academy of Science (UCAS), Beijing, China

${ }^{16}$ Institute of Physics, University of Belgrade, Belgrade, Serbia

${ }^{17}$ Department for Physics and Technology, University of Bergen, Bergen, Norway

${ }^{18}$ Physics Division, Lawrence Berkeley National Laboratory and University of California, Berkeley, CA, USA

${ }^{19}$ Institut für Physik, Humboldt Universität zu Berlin, Berlin, Germany

${ }^{20}$ Albert Einstein Center for Fundamental Physics and Laboratory for High Energy Physics, University of Bern, Bern, Switzerland

${ }^{21}$ School of Physics and Astronomy, University of Birmingham, Birmingham, UK

22 (a) Facultad de Ciencias y Centro de Investigaciónes, Universidad Antonio Nariño, Bogotá, Colombia; ${ }^{(b)}$ Departamento de Física, Universidad Nacional de Colombia, Bogotá, Colombia

23 (a) Dipartimento di Fisica, INFN Bologna and Universita' di Bologna, Bologna, Italy; ${ }^{(b)}$ INFN Sezione di Bologna, Bologna, Italy

${ }^{24}$ Physikalisches Institut, Universität Bonn, Bonn, Germany

${ }^{25}$ Department of Physics, Boston University, Boston, MA, USA

${ }^{26}$ Department of Physics, Brandeis University, Waltham, MA, USA

27 (a) Transilvania University of Brasov, Brasov, Romania; (b) Horia Hulubei National Institute of Physics and Nuclear Engineering, Bucharest, Romania; ${ }^{(c)}$ Department of Physics, Alexandru Ioan Cuza University of Iasi, Iasi, Romania; ${ }^{(d)}$ National Institute for Research and Development of Isotopic and Molecular Technologies, Physics Department, Cluj-Napoca, Romania; ${ }^{(\mathrm{e})}$ University Politehnica Bucharest, Bucharest, Romania; ${ }^{(\mathrm{f})}$ West University in Timisoara, Timisoara, Romania

28 (a) Faculty of Mathematics, Physics and Informatics, Comenius University, Bratislava, Slovak Republic; ${ }^{(b)}$ Department of Subnuclear Physics, Institute of Experimental Physics of the Slovak Academy of Sciences, Kosice, Slovak Republic

${ }^{29}$ Physics Department, Brookhaven National Laboratory, Upton, NY, USA

${ }^{30}$ Departamento de Física, Universidad de Buenos Aires, Buenos Aires, Argentina

${ }^{31}$ California State University, Long Beach, CA, USA

${ }^{32}$ Cavendish Laboratory, University of Cambridge, Cambridge, UK

33 (a) Department of Physics, University of Cape Town, Cape Town, South Africa; ${ }^{(b)}$ iThemba Labs, Western Cape, South Africa; ${ }^{(c)}$ Department of Mechanical Engineering Science, University of Johannesburg, Johannesburg, South Africa; (d) University of South Africa, Department of Physics, Pretoria, South Africa; ${ }^{(e)}$ School of Physics, University of the Witwatersrand, Johannesburg, South Africa

${ }^{34}$ Department of Physics, Carleton University, Ottawa, ON, Canada

35 (a) Faculté des Sciences Ain Chock, Réseau Universitaire de Physique des Hautes Energies - Université Hassan II, Casablanca, Morocco; ${ }^{(b)}$ Faculté des Sciences, Université Ibn-Tofail, Kénitra, Morocco; ${ }^{(c)}$ Faculté des Sciences Semlalia, Université Cadi Ayyad, LPHEA-Marrakech, Morocco; ${ }^{(d)}$ Moroccan Foundation for Advanced Science Innovation and Research (MAScIR), Rabat, Morocco; ${ }^{(e)}$ LPMR, Faculté des Sciences, Université Mohamed Premier, Oujda, Morocco; ${ }^{(\mathrm{f})}$ Faculté des sciences, Université Mohammed V, Rabat, Morocco

${ }^{36}$ CERN, Geneva, Switzerland

${ }^{37}$ Enrico Fermi Institute, University of Chicago, Chicago, IL, USA

${ }^{38}$ LPC, Université Clermont Auvergne, CNRS/IN2P3, Clermont-Ferrand, France

${ }^{39}$ Nevis Laboratory, Columbia University, Irvington, NY, USA

${ }^{40}$ Niels Bohr Institute, University of Copenhagen, Copenhagen, Denmark

41 (a) Dipartimento di Fisica, Università della Calabria, Rende, Italy; ${ }^{(b)}$ Laboratori Nazionali di Frascati, INFN Gruppo Collegato di Cosenza, Frascati, Italy

42 Physics Department, Southern Methodist University, Dallas, TX, USA 
${ }^{43}$ Physics Department, University of Texas at Dallas, Richardson, TX, USA

${ }^{44}$ National Centre for Scientific Research "Demokritos", Agia Paraskevi, Greece

45 (a) Department of Physics, Stockholm University, Stockholm, Sweden; ${ }^{(b)}$ Oskar Klein Centre, Stockholm, Sweden

${ }^{46}$ Deutsches Elektronen-Synchrotron DESY, Hamburg and Zeuthen, Germany

${ }^{47}$ Lehrstuhl für Experimentelle Physik IV, Technische Universität Dortmund, Dortmund, Germany

${ }^{48}$ Institut für Kern- und Teilchenphysik, Technische Universität Dresden, Dresden, Germany

${ }^{49}$ Department of Physics, Duke University, Durham, NC, USA

${ }^{50}$ SUPA-School of Physics and Astronomy, University of Edinburgh, Edinburgh, UK

${ }^{51}$ INFN e Laboratori Nazionali di Frascati, Frascati, Italy

${ }^{52}$ Physikalisches Institut, Albert-Ludwigs-Universität Freiburg, Freiburg, Germany

${ }^{53}$ II. Physikalisches Institut, Georg-August-Universität Göttingen, Göttingen, Germany

${ }^{54}$ Département de Physique Nucléaire et Corpusculaire, Université de Genève, Geneva, Switzerland

55 (a) Dipartimento di Fisica, Università di Genova, Genoa, Italy; ${ }^{(b)}$ INFN Sezione di Genova, Genoa, Italy

${ }^{56}$ II. Physikalisches Institut, Justus-Liebig-Universität Giessen, Giessen, Germany

${ }^{57}$ SUPA-School of Physics and Astronomy, University of Glasgow, Glasgow, UK

${ }^{58}$ LPSC, Université Grenoble Alpes, CNRS/IN2P3, Grenoble INP, Grenoble, France

${ }^{59}$ Laboratory for Particle Physics and Cosmology, Harvard University, Cambridge, MA, USA

60 (a) State Key Laboratory of Particle Detection and Electronics, Department of Modern Physics, University of Science and Technology of China, Hefei, China; ${ }^{(b)}$ Key Laboratory of Particle Physics and Particle Irradiation (MOE), Institute of Frontier and Interdisciplinary Science, Shandong University, Qingdao, China; (c) School of Physics and Astronomy, Shanghai Jiao Tong University, Key Laboratory for Particle Astrophysics and Cosmology (MOE), SKLPPC, Shanghai, China; ${ }^{(d)}$ Tsung-Dao Lee Institute, Shanghai, China

61 (a) Kirchhoff-Institut für Physik, Ruprecht-Karls-Universität Heidelberg, Heidelberg, Germany; ${ }^{(\mathrm{b})}$ Physikalisches Institut, Ruprecht-Karls-Universität Heidelberg, Heidelberg, Germany

62 Faculty of Applied Information Science, Hiroshima Institute of Technology, Hiroshima, Japan

63 (a) Department of Physics, Chinese University of Hong Kong, Shatin N.T., Hong Kong; (b) Department of Physics, University of Hong Kong, Pok Fu Lam, Hong Kong; ${ }^{\left({ }^{c}\right)}$ Department of Physics and Institute for Advanced Study, Hong Kong University of Science and Technology, Clear Water Bay, Kowloon, Hong Kong, China

${ }^{64}$ Department of Physics, National Tsing Hua University, Hsinchu, Taiwan

${ }^{65}$ IJCLab, CNRS/IN2P3, Université Paris-Saclay, 91405 Orsay, France

${ }^{66}$ Department of Physics, Indiana University, Bloomington, IN, USA

67 (a) INFN Gruppo Collegato di Udine, Sezione di Trieste, Udine, Italy; ${ }^{(b)}$ ICTP, Trieste, Italy; ${ }^{(c)}$ Dipartimento Politecnico di Ingegneria e Architettura, Università di Udine, Udine, Italy

68 (a) INFN Sezione di Lecce, Lecce, Italy; ${ }^{\left({ }^{b}\right)}$ Dipartimento di Matematica e Fisica, Università del Salento, Lecce, Italy

69 (a) INFN Sezione di Milano, Milan, Italy; ${ }^{(b)}$ Dipartimento di Fisica, Università di Milano, Milan, Italy

70 (a) INFN Sezione di Napoli, Naples, Italy; ${ }^{(b)}$ Dipartimento di Fisica, Università di Napoli, Naples, Italy

71 (a) INFN Sezione di Pavia, Pavia, Italy; ${ }^{(b)}$ Dipartimento di Fisica, Università di Pavia, Pavia, Italy

72 (a) INFN Sezione di Pisa, Pisa, Italy; ${ }^{\text {(b) }}$ Dipartimento di Fisica E. Fermi, Università di Pisa, Pisa, Italy

73 (a) INFN Sezione di Roma, Rome, Italy; ${ }^{(b)}$ Dipartimento di Fisica, Sapienza Università di Roma, Rome, Italy

74 (a) INFN Sezione di Roma Tor Vergata, Rome, Italy; ${ }^{(b)}$ Dipartimento di Fisica, Università di Roma Tor Vergata, Rome, Italy

75 (a) INFN Sezione di Roma Tre, Rome, Italy; ${ }^{(b)}$ Dipartimento di Matematica e Fisica, Università Roma Tre, Rome, Italy

76 (a) INFN-TIFPA, Trento, Italy; (b) Università degli Studi di Trento, Trento, Italy

${ }^{77}$ Institut für Astro- und Teilchenphysik, Leopold-Franzens-Universität, Innsbruck, Austria

${ }^{78}$ University of Iowa, Iowa City, IA, USA

${ }^{79}$ Department of Physics and Astronomy, Iowa State University, Ames, IA, USA

${ }^{80}$ Joint Institute for Nuclear Research, Dubna, Russia

81 (a) Departamento de Engenharia Elétrica, Universidade Federal de Juiz de Fora (UFJF), Juiz de Fora,

Brazil; ${ }^{\left({ }^{b}\right)}$ Universidade Federal do Rio De Janeiro COPPE/EE/IF, Rio de Janeiro, Brazil; ${ }^{(c)}$ Instituto de Física,

Universidade de São Paulo, São Paulo, Brazil

${ }^{82}$ KEK, High Energy Accelerator Research Organization, Tsukuba, Japan

${ }^{83}$ Graduate School of Science, Kobe University, Kobe, Japan

84 (a) AGH University of Science and Technology, Faculty of Physics and Applied Computer Science, Kraków, 
Poland; ${ }^{(b)}$ Marian Smoluchowski Institute of Physics, Jagiellonian University, Kraków, Poland

${ }^{85}$ Institute of Nuclear Physics Polish Academy of Sciences, Kraków, Poland

${ }^{86}$ Faculty of Science, Kyoto University, Kyoto, Japan

${ }^{87}$ Kyoto University of Education, Kyoto, Japan

${ }^{88}$ Research Center for Advanced Particle Physics and Department of Physics, Kyushu University, Fukuoka, Japan

${ }^{89}$ Instituto de Física La Plata, Universidad Nacional de La Plata and CONICET, La Plata, Argentina

${ }^{90}$ Physics Department, Lancaster University, Lancaster, UK

${ }^{91}$ Oliver Lodge Laboratory, University of Liverpool, Liverpool, UK

92 Department of Experimental Particle Physics, Jožef Stefan Institute and Department of Physics, University of Ljubljana, Ljubljana, Slovenia

${ }^{93}$ School of Physics and Astronomy, Queen Mary University of London, London, UK

${ }^{94}$ Department of Physics, Royal Holloway University of London, Egham, UK

${ }^{95}$ Department of Physics and Astronomy, University College London, London, UK

${ }^{96}$ Louisiana Tech University, Ruston, LA, USA

${ }^{97}$ Fysiska institutionen, Lunds universitet, Lund, Sweden

${ }^{98}$ Centre de Calcul de l'Institut National de Physique Nucléaire et de Physique des Particules (IN2P3), Villeurbanne, France

${ }^{99}$ Departamento de Física Teorica C-15 and CIAFF, Universidad Autónoma de Madrid, Madrid, Spain

100 Institut für Physik, Universität Mainz, Mainz, Germany

${ }^{101}$ School of Physics and Astronomy, University of Manchester, Manchester, UK

102 CPPM, Aix-Marseille Université, CNRS/IN2P3, Marseille, France

103 Department of Physics, University of Massachusetts, Amherst, MA, USA

${ }^{104}$ Department of Physics, McGill University, Montreal, QC, Canada

105 School of Physics, University of Melbourne, Victoria, Australia

106 Department of Physics, University of Michigan, Ann Arbor, MI, USA

${ }^{107}$ Department of Physics and Astronomy, Michigan State University, East Lansing, MI, USA

108 B.I. Stepanov Institute of Physics, National Academy of Sciences of Belarus, Minsk, Belarus

${ }^{109}$ Research Institute for Nuclear Problems of Byelorussian State University, Minsk, Belarus

${ }^{110}$ Group of Particle Physics, University of Montreal, Montreal, QC, Canada

${ }^{111}$ P.N. Lebedev Physical Institute of the Russian Academy of Sciences, Moscow, Russia

${ }^{112}$ National Research Nuclear University MEPhI, Moscow, Russia

${ }^{113}$ D.V. Skobeltsyn Institute of Nuclear Physics, M.V. Lomonosov Moscow State University, Moscow, Russia

${ }^{114}$ Fakultät für Physik, Ludwig-Maximilians-Universität München, Munich, Germany

115 Max-Planck-Institut für Physik (Werner-Heisenberg-Institut), Munich, Germany

116 Nagasaki Institute of Applied Science, Nagasaki, Japan

${ }^{117}$ Graduate School of Science and Kobayashi-Maskawa Institute, Nagoya University, Nagoya, Japan

${ }^{118}$ Department of Physics and Astronomy, University of New Mexico, Albuquerque, NM, USA

${ }^{119}$ Institute for Mathematics, Astrophysics and Particle Physics, Radboud University/Nikhef, Nijmegen, The Netherlands

${ }^{120}$ Nikhef National Institute for Subatomic Physics and University of Amsterdam, Amsterdam, The Netherlands

${ }^{121}$ Department of Physics, Northern Illinois University, DeKalb, IL, USA

122 (a) Budker Institute of Nuclear Physics and NSU, SB RAS, Novosibirsk, Russia; ${ }^{(b)}$ Novosibirsk State University

Novosibirsk, Novosibirsk, Russia

${ }^{123}$ Institute for High Energy Physics of the National Research Centre Kurchatov Institute, Protvino, Russia

${ }^{124}$ Institute for Theoretical and Experimental Physics named by A.I. Alikhanov of National Research Centre "Kurchatov Institute", Moscow, Russia

125 Department of Physics, New York University, New York, NY, USA

${ }^{126}$ Ochanomizu University, Otsuka, Bunkyo-ku, Tokyo, Japan

127 Ohio State University, Columbus, OH, USA

${ }^{128}$ Homer L. Dodge Department of Physics and Astronomy, University of Oklahoma, Norman, OK, USA

${ }^{129}$ Department of Physics, Oklahoma State University, Stillwater, OK, USA

${ }^{130}$ RCPTM, Joint Laboratory of Optics, Palacký University, Olomouc, Czech Republic

${ }^{131}$ Institute for Fundamental Science, University of Oregon, Eugene, OR, USA

${ }^{132}$ Graduate School of Science, Osaka University, Osaka, Japan 
133 Department of Physics, University of Oslo, Oslo, Norway

134 Department of Physics, Oxford University, Oxford, UK

${ }^{135}$ LPNHE, CNRS/IN2P3, Sorbonne Université, Université de Paris, Paris, France

136 Department of Physics, University of Pennsylvania, Philadelphia, PA, USA

${ }^{137}$ Konstantinov Nuclear Physics Institute of National Research Centre "Kurchatov Institute", PNPI, St. Petersburg, Russia

${ }^{138}$ Department of Physics and Astronomy, University of Pittsburgh, Pittsburgh, PA, USA

139 (a) Laboratório de Instrumentação e Física Experimental de Partículas-LIP, Lisbon, Portugal; ${ }^{(b)}$ Departamento de Física, Faculdade de Ciências, Universidade de Lisboa, Lisbon, Portugal; ${ }^{(c)}$ Departamento de Física, Universidade de Coimbra, Coimbra, Portugal; ${ }^{(d)}$ Centro de Física Nuclear da Universidade de Lisboa, Lisbon, Portugal; ${ }^{(e)}$ Departamento de Física, Universidade do Minho, Braga, Portugal; ${ }^{(\mathrm{f})}$ Departamento de Física Teórica y del Cosmos, Universidad de Granada, Granada, Spain; ${ }^{(\mathrm{g})}$ Dep Física and CEFITEC of Faculdade de Ciências e Tecnologia, Universidade Nova de Lisboa, Caparica, Portugal; ${ }^{(h)}$ Instituto Superior Técnico, Universidade de Lisboa, Lisbon, Portugal

${ }^{140}$ Institute of Physics of the Czech Academy of Sciences, Prague, Czech Republic

${ }^{141}$ Czech Technical University in Prague, Prague, Czech Republic

${ }^{142}$ Charles University, Faculty of Mathematics and Physics, Prague, Czech Republic

143 Particle Physics Department, Rutherford Appleton Laboratory, Didcot, UK

144 IRFU, CEA, Université Paris-Saclay, Gif-sur-Yvette, France

${ }^{145}$ Santa Cruz Institute for Particle Physics, University of California Santa Cruz, Santa Cruz, CA, USA

146 (a) Departamento de Física, Pontificia Universidad Católica de Chile, Santiago, Chile; (b) Universidad Andres Bello,

Department of Physics, Santiago, Chile; (c) Instituto de Alta Investigación, Universidad de Tarapacá, Arica,

Chile; ${ }^{(d)}$ Departamento de Física, Universidad Técnica Federico Santa María, Valparaiso, Chile

${ }^{147}$ Universidade Federal de São João del Rei (UFSJ), São João del Rei, Brazil

148 Department of Physics, University of Washington, Seattle, WA, USA

${ }^{149}$ Department of Physics and Astronomy, University of Sheffield, Sheffield, UK

${ }^{150}$ Department of Physics, Shinshu University, Nagano, Japan

${ }^{151}$ Department Physik, Universität Siegen, Siegen, Germany

152 Department of Physics, Simon Fraser University, Burnaby, BC, Canada

${ }^{153}$ SLAC National Accelerator Laboratory, Stanford, CA, USA

${ }^{154}$ Physics Department, Royal Institute of Technology, Stockholm, Sweden

155 Departments of Physics and Astronomy, Stony Brook University, Stony Brook, NY, USA

${ }^{156}$ Department of Physics and Astronomy, University of Sussex, Brighton, UK

${ }^{157}$ School of Physics, University of Sydney, Sydney, Australia

158 Institute of Physics, Academia Sinica, Taipei, Taiwan

159 (a) E. Andronikashvili Institute of Physics, Iv. Javakhishvili Tbilisi State University, Tbilisi, Georgia; ${ }^{(b)}$ High Energy

Physics Institute, Tbilisi State University, Tbilisi, Georgia

${ }^{160}$ Department of Physics, Technion, Israel Institute of Technology, Haifa, Israel

${ }^{161}$ Raymond and Beverly Sackler School of Physics and Astronomy, Tel Aviv University, Tel Aviv, Israel

162 Department of Physics, Aristotle University of Thessaloniki, Thessaloníki, Greece

${ }^{163}$ International Center for Elementary Particle Physics and Department of Physics, University of Tokyo, Tokyo, Japan

${ }^{164}$ Graduate School of Science and Technology, Tokyo Metropolitan University, Tokyo, Japan

165 Department of Physics, Tokyo Institute of Technology, Tokyo, Japan

166 Tomsk State University, Tomsk, Russia

167 Department of Physics, University of Toronto, Toronto, ON, Canada

168 (a) TRIUMF, Vancouver, BC, Canada; ${ }^{(b)}$ Department of Physics and Astronomy, York University, Toronto, ON, Canada

${ }^{169}$ Division of Physics and Tomonaga Center for the History of the Universe, Faculty of Pure and Applied Sciences,

University of Tsukuba, Tsukuba, Japan

${ }^{170}$ Department of Physics and Astronomy, Tufts University, Medford, MA, USA

${ }^{171}$ Department of Physics and Astronomy, University of California Irvine, Irvine, CA, USA

172 Department of Physics and Astronomy, University of Uppsala, Uppsala, Sweden

${ }^{173}$ Department of Physics, University of Illinois, Urbana, IL, USA

${ }^{174}$ Instituto de Física Corpuscular (IFIC), Centro Mixto Universidad de Valencia - CSIC, Valencia, Spain

175 Department of Physics, University of British Columbia, Vancouver, BC, Canada

${ }^{176}$ Department of Physics and Astronomy, University of Victoria, Victoria, BC, Canada 
${ }^{177}$ Fakultät für Physik und Astronomie, Julius-Maximilians-Universität Würzburg, Würzburg, Germany

178 Department of Physics, University of Warwick, Coventry, UK

${ }^{179}$ Waseda University, Tokyo, Japan

${ }^{180}$ Department of Particle Physics and Astrophysics, Weizmann Institute of Science, Rehovot, Israel

${ }^{181}$ Department of Physics, University of Wisconsin, Madison, WI, USA

182 Fakultät für Mathematik und Naturwissenschaften, Fachgruppe Physik, Bergische Universität Wuppertal, Wuppertal, Germany

${ }^{183}$ Department of Physics, Yale University, New Haven, CT, USA

${ }^{a}$ Also at Borough of Manhattan Community College, City University of New York, New York, NY, USA

${ }^{\mathrm{b}}$ Also at Center for High Energy Physics, Peking University, China

${ }^{c}$ Also at Centro Studi e Ricerche Enrico Fermi, Rome, Italy

d Also at CERN, Geneva, Switzerland

e Also at CPPM, Aix-Marseille Université, CNRS/IN2P3, Marseille, France

${ }^{\mathrm{f}}$ Also at Département de Physique Nucléaire et Corpusculaire, Université de Genève, Geneva, Switzerland

g Also at Departament de Fisica de la Universitat Autonoma de Barcelona, Barcelona, Spain

${ }^{\mathrm{h}}$ Also at Department of Financial and Management Engineering, University of the Aegean, Chios, Greece

${ }^{\mathrm{i}}$ Also at Department of Physics and Astronomy, Michigan State University, East Lansing, MI, USA

${ }^{\mathrm{j}}$ Also at Department of Physics and Astronomy, University of Louisville, Louisville, KY, USA

${ }^{k}$ Also at Department of Physics, Ben Gurion University of the Negev, Beer Sheva, Israel

${ }^{1}$ Also at Department of Physics, California State University, East Bay, USA

m Also at Department of Physics, California State University, Fresno, USA

${ }^{\mathrm{n}}$ Also at Department of Physics, California State University, Sacramento, USA

${ }^{\circ}$ Also at Department of Physics, King's College London, London, UK

p Also at Department of Physics, St. Petersburg State Polytechnical University, St. Petersburg, Russia

q Also at Department of Physics, University of Fribourg, Fribourg, Switzerland

${ }^{\mathrm{r}}$ Also at Dipartimento di Matematica, Informatica e Fisica, Università di Udine, Udine, Italy

${ }^{s}$ Also at Faculty of Physics, M.V. Lomonosov Moscow State University, Moscow, Russia

${ }^{\mathrm{t}}$ Also at Giresun University, Faculty of Engineering, Giresun, Turkey

u Also at Graduate School of Science, Osaka University, Osaka, Japan

${ }^{v}$ Also at Hellenic Open University, Patras, Greece

${ }^{w}$ Also at Institucio Catalana de Recerca i Estudis Avancats, ICREA, Barcelona, Spain

x Also at Institut für Experimentalphysik, Universität Hamburg, Hamburg, Germany

y Also at Institute for Nuclear Research and Nuclear Energy (INRNE) of the Bulgarian Academy of Sciences, Sofia, Bulgaria

${ }^{\mathrm{z}}$ Also at Institute for Particle and Nuclear Physics, Wigner Research Centre for Physics, Budapest, Hungary

aa Also at Institute of Particle Physics (IPP), Montreal, Canada

${ }^{a b}$ Also at Institute of Physics, Azerbaijan Academy of Sciences, Baku, Azerbaijan

${ }^{a c}$ Also at Instituto de Fisica Teorica, IFT-UAM/CSIC, Madrid, Spain

${ }^{\text {ad }}$ Also at Dept. of Physics, Istanbul University, Istanbul, Turkey

ae Also at Joint Institute for Nuclear Research, Dubna, Russia

${ }^{\text {af }}$ Also at Moscow Institute of Physics and Technology State University, Dolgoprudny, Russia

ag Also at National Research Nuclear University MEPhI, Moscow, Russia

ah Also at Physics Department, An-Najah National University, Nablus, Palestine

ai Also at Physikalisches Institut, Albert-Ludwigs-Universität Freiburg, Freiburg, Germany

aj Also at The City College of New York, New York, NY, USA

${ }^{\text {ak }}$ Also at TRIUMF, Vancouver, BC, Canada

${ }^{\text {al }}$ Also at Universita di Napoli Parthenope, Naples, Italy

${ }^{a m}$ Also at University of Chinese Academy of Sciences (UCAS), Beijing, China

* Deceased 